Informaatioteknologian tiedekunnan julkaisuja

No. 27/2016

Pekka Neittaanmäki, Elmira Galeieva, Anthony Ogbechie

\title{
Transportation Technologies
}

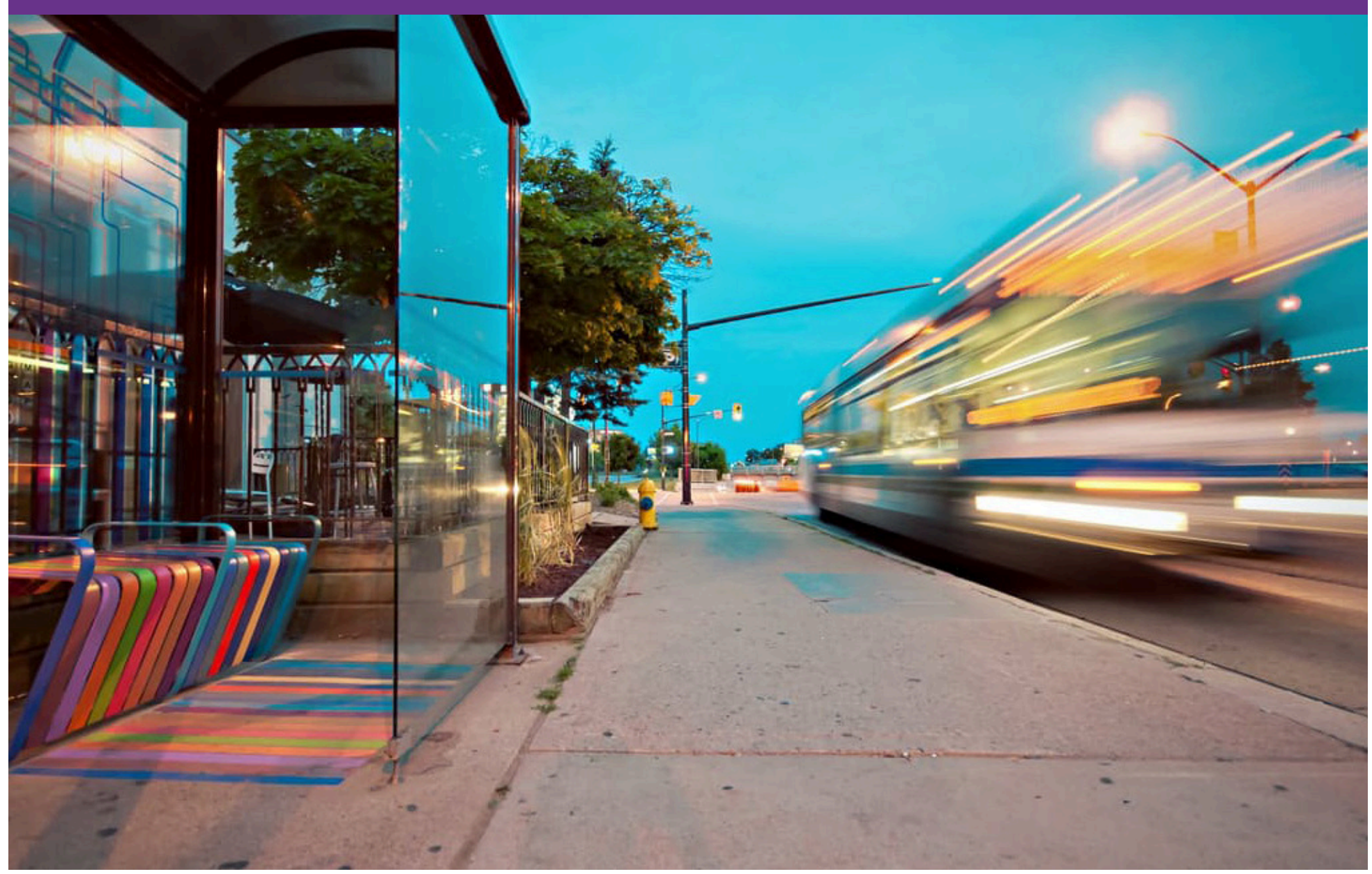


Informaatioteknologian tiedekunnan julkaisuja

No. 27/2016

Editor: Pekka Neittaanmäki

Covers: Jarno Kiesiläinen

Copyright (C) 2016

Pekka Neittaanmäki, Elmira Galeieva, Anthony Ogbechie ja Jyväskylän yliopisto

ISBN 978-951-39-6925-7 (verkkoj.)

ISSN 2323-5004

Jyväskylä 2016 


\title{
Transportation Technologies
}

Pekka Neittaanmäki, Elmira Galeieva, Anthony Ogbechie

\begin{abstract}
ABOUT THE PROJECT
This report was conducted under the Platform Value Now project funded by Finland's Strategic Research Council. Platform Value Now will focus on understanding the fast emerging platform ecosystems, their value creation dynamics and requirements of the supportive institutional environment. We will analyze ecosystems with systems tools and develop new methods for platform-centric ecosystems management. Data collection is based on active scanning of global technology and platform ecosystems and fast solution oriented case experiments with Finnish corporations and policy planners. The aim of the project is to operationalize the collected understanding into a Platform Profile framework that will enable more efficient method and tool development for ecosystem management.
\end{abstract}




\section{Content}

Large-Scale Signal Optimization: Traffic Platform Framework and Applications.. 7

Traffic congestion detection in large-scale scenarios using vehicle-to-vehicle communications

Diverse Large-Scale ITS Dataset Created from Continuous Learning for RealTime Vehicle Detection

City Management Platform Using Big Data from People and Traffic Flows. 8

NTT DATA SOLVING TRAFFIC CONGESTION AND ENABLING SMARTER CITIES USING BIG DATA ANALYTICS.

SMaRTCaR: An integrated smartphone-based platform to support traffic management applications

Intelligent Transportation Systems (ITS) Operations................................. 10

Intelligent Transportation Systems ......................................................... 10

Mobile phone location determination and its impact on intelligent transportation systems

SUMMARY OF VEHICLE DETECTION AND SURVEILLANCE TECHNOLOGIES USED IN INTELLIGENT TRANSPORTATION SYSTEMS ............................. 11

Toward the development of intelligent transportation systems ........................ 12

Parallel Control and Management for Intelligent Transportation Systems:

Concepts, Architectures, and Applications ............................................. 12

A Survey on Intelligent Transportation Systems......................................... 13

Performance Analysis of PMIPv6-Based NEtwork MObility for Intelligent

Transportation Systems

Day-to-day dynamic models for Intelligent Transportation Systems design and appraisal.

Optimization models for assessing the peak capacity utilization of intelligent

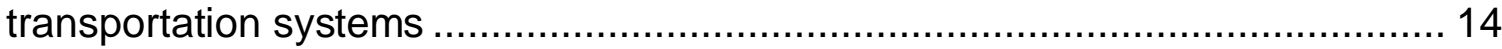

CrowdITS: Crowdsourcing in intelligent transportation systems.................... 15

Intelligent transportation systems for smart cities: a progress review............... 15

A review on vision-based pedestrian detection in intelligent transportation

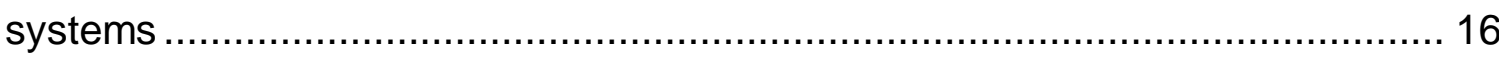

Meeting privacy challenges while advancing intelligent transportation systems. 16 Context-Aware Driver Behavior Detection System in Intelligent Transportation Systems 
Research and Development of Intelligent Transportation Systems

A multi-agent approach to Intelligent Transportation Systems modeling with combinatorial auctions.

Sensor and network technology for intelligent transportation systems 18

Recent trends in intelligent transportation systems: a review ......................... 19

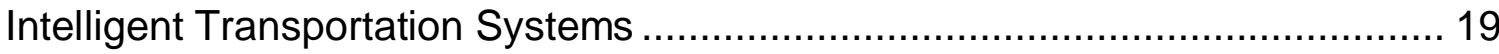

A Comparative Study of Three Multivariate Short-Term Freeway Traffic Flow Forecasting Methods With Missing Data.

Build intelligent transportation systems with the Traffic Management Data Dictionary standard 20

Practical Camera Calibration From Moving Objects for Traffic Scene Surveillance

Advanced Urban Traffic Management System. 21

Sustainable Transportation Ecosystem Addressing sustainability from an integrated systems perspective 21

Digital-Age Transportation. The Future of Urban Mobility ............................... 22

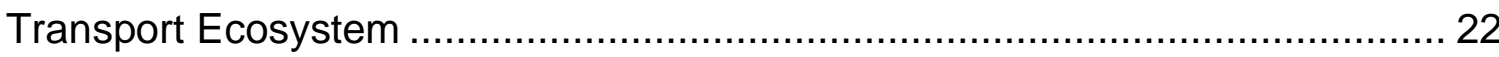

The future of mobility. How transportation technology and social trends are creating a new business ecosystem.................................................. 23

Where is public transportation going? Insights from the high performers .......... 23

Why the Transportation Industry is Getting on Board with Big Data \& Hadoop.. 24

Big Data's Implications for Transportation Operations. An Exploration .............. 24

Maximizing Returns through Advanced Analytics in Transportation................... 25

Transport Intermodality: Integrated Transport for the 21st Century.................. 25

Intelligent transport management system for urban traffic hubs based on an integration of multiple technologies ............................................................. 26

Assessing the Potential Impacts of Connected Vehicles: Mobility, Environmental, and Safety Perspectives. 26

Comparison of In-Vehicle Auditory Public Traffic Information With Roadside Dynamic Message Signs

Parking Spaces Repurchase Strategy Design via Simulation Optimization ....... 27

Urban Traffic Flow Prediction Using a Spatio-Temporal Random Effects Model 28 Dynamic Bandwidth Analysis for Coordinated Arterial Streets ........................ 28

Incident management in intelligent transportation systems .......................... 29 
Intelligent Transportation Systems: New Principles and Architectures............... 29

Intelligent Transportation Systems Architectures .......................................... 30

Human Factors in Intelligent Transportation Systems .................................... 30

Positioning Systems in Intelligent Transportation Systems .............................. 31

Integrated Platform for Road Traffic Safety Data Collection and Information

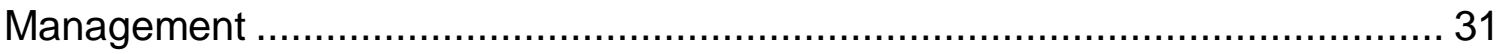

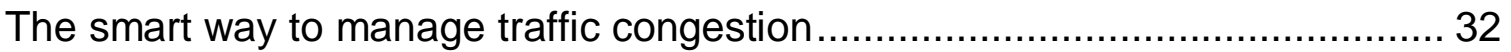

How digital road management could save the UK £300bn by 2030 .................. 32

Departure Side Platforms: a road congestion mitigation measure ....................... 33

Advanced Data Analytics in Transport - Machine Learning Perspective ........... 33

MEMS sensor solutions for loT in transport and buildings .............................. 34

Digital platform more than taxi service: Uber as app economy paradigm?......... 34

First Steps toward Drone Traffic Management ................................................ 35

A framework for artificial transportation systems: From computer simulations to

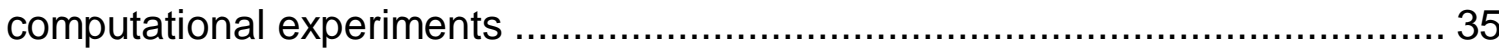

Real Time Simulation Platform Design of Traffic Control Hardware In-The-Loop

Emerging transport technologies: Assessing impacts and implications for the City of Melbourne 36

Predictable Disruption: Looking to digital ecosystems for the next waves of change

Relieving Traffic Congestion by Tracking Vehicle Movement: Leveraging Locus Data to Help Make Roads Safer and Better ................................................... 38

Transforming transport by building ecosystems, not ego-systems .................... 38

Better transportation customer experiences through digital ............................. 39

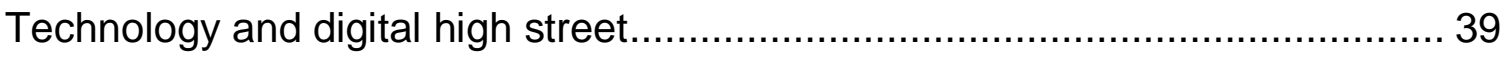

Self-driving cars are about platforms, not about cars ..................................... 40

Research on the Platform Construction of Road Freight Market in China.......... 40 A WIDER SHARING ECOSYSTEM The pivotal role of data in transport solutions

Data61 is building a transport analytics platform for the states 41

TIMON ("Enhanced real time services for an optimized multimodal mobility relying on cooperative networks and open data")........................................ 42 Innovative Transport Services in Finland ..................................................... 42 
Navigating a Shifting Landscape: Capturing Value in the Evolving Mobility Ecosystem.

THE ROLE OF TRANSPORT TECHNOLOGY PLATFORMS IN FOSTERING EXPLOITATION

The Emergence of the German Digital Autobahn Ecosystem .......................... 44

The Coming Fourth Digital Revolution in the Travel Ecosystem ....................... 44

Transport for London creates an open data ecosystem with Amazon Web

Services

Business Strategy: Harnessing Connected Vehicle Ecosystem B2X Opportunities

Apps for transport maps: connecting the dots in a complex ecosystem ............. 46

Smart communication for intelligent transport ................................................ 46

The New Transportation Ecosystem and the Role of Silicon Valley................... 47

What the rise of the sharing economy means for transport .............................. 47

Revolutionizing the Travel and Transportation Ecosystem............................... 48

How Technology is Changing Traffic Reporting …........................................ 48

Arctic testing ecosystem for intelligent transport opens in Finnish Lapland........ 49

Intelligent transport - a trump card for Finnish exports ................................... 49

Challenges to Urban Transport Sustainability and Smart Transport in a Tourist

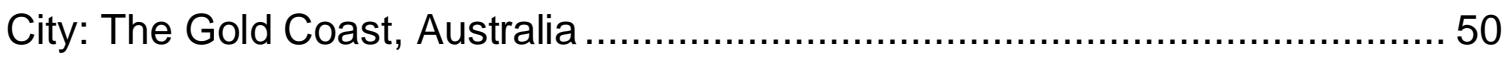

Urban Ecosystems: Roads and Transits .................................................... 50

DIGITAL TRANSFORMATIONS IMPACTING THE ECOSYSTEM OF FLIGHT 51

On Multi-Agent Based Urban Rail Transport Ridership Forecast System .......... 51

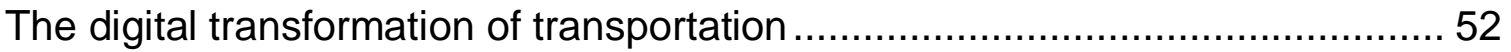

Routes to prosperity: How smart transport infrastructure can help cities to thrive

Intelligent Urban Exchange - Intelligent Transport Transportation \& Mobility - Bus

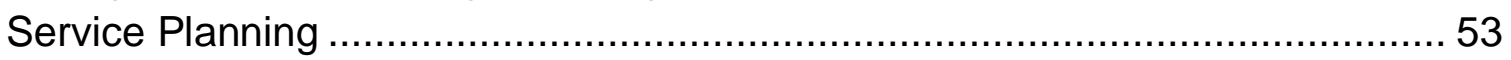

A system dynamic approach to planning of resilient urban systems .................. 53

Can the 'internet of moving things' end traffic jams? ........................................ 54

City transport needs saving from itself - here's how to do it ........................... 54

Microscopic dynamic simulation model for pedestrian-vehicle mixed traffic....... 55

Digital Technology, Mobility And Convenience Services: What Are The

Challenges When Building The Collaborative City Of The Future? 
An Architectural Framework and Enabling Wireless Technologies for Digital Cities \& Intelligent Urban Environments. 56

MaaS Finland aims to revolutionize the global transportation market 57 


\section{Large-Scale Signal Optimization: Traffic Platform Framework and Applications}

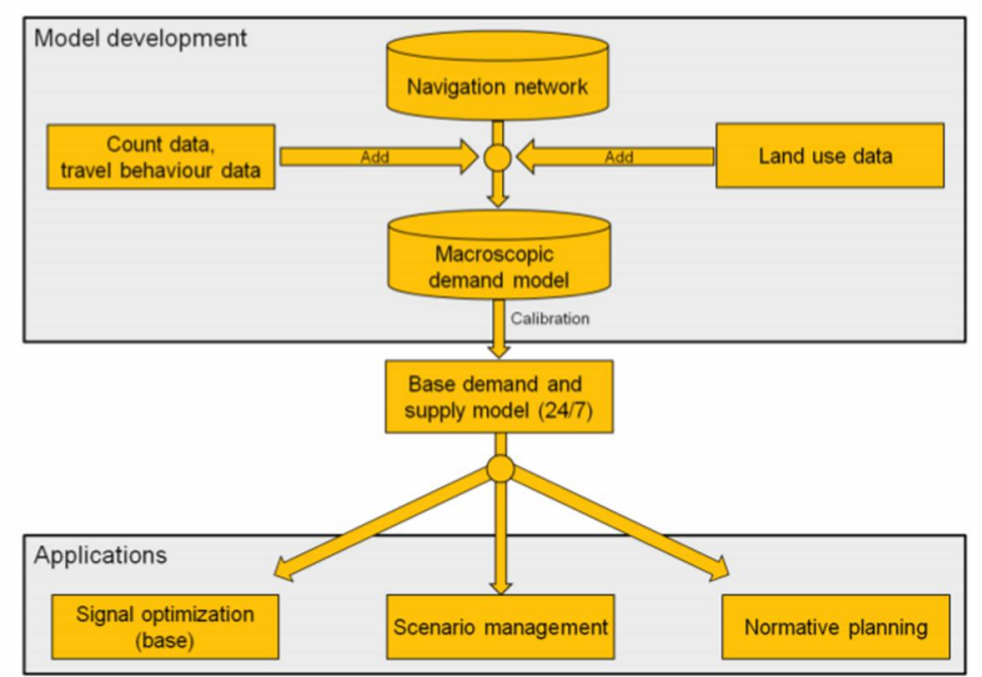

Read Article:

http://onlinepubs.trb.org/onlinepubs/conferences/2012/4thITM/Papers-R/0117000066.pdf

Traffic congestion detection in large-scale scenarios using vehicle-to-vehicle communications

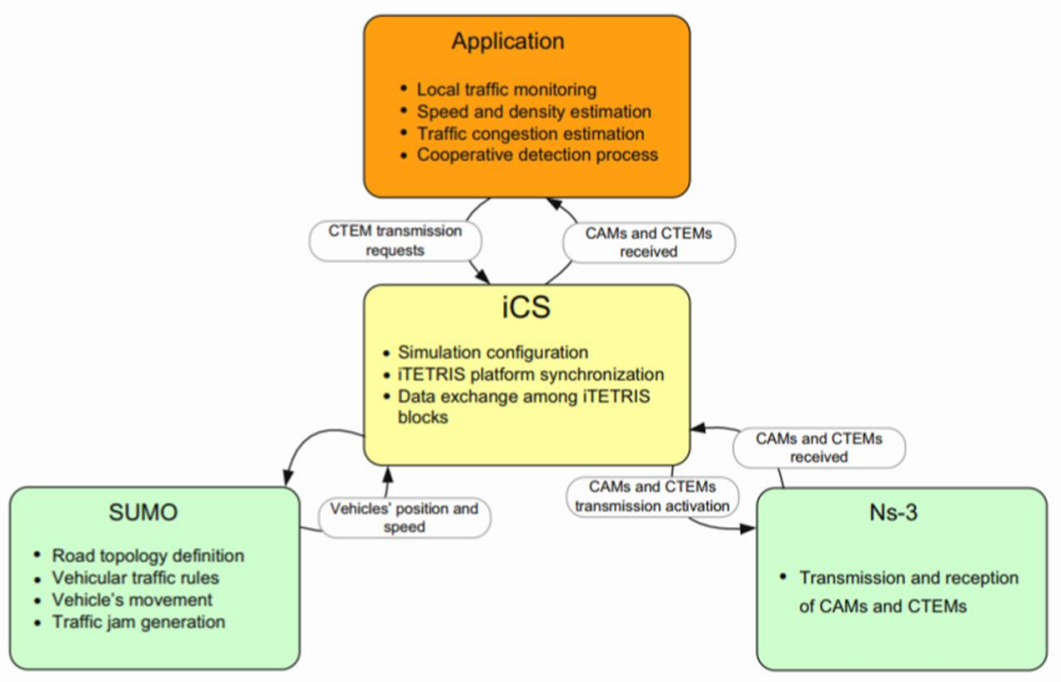

Read Article:

http://ac.els-cdn.com/S1084804512000628/1-s2.0-S1084804512000628-

main.pdf?_tid=4742b5f8-1a1b-11e6-ba3e-

00000aacb360\&acdnat $=1463261712$ bb755b3f924f2b65d1b5e46ef2d2ced 1 
Diverse Large-Scale ITS Dataset Created from Continuous Learning for Real-Time Vehicle Detection

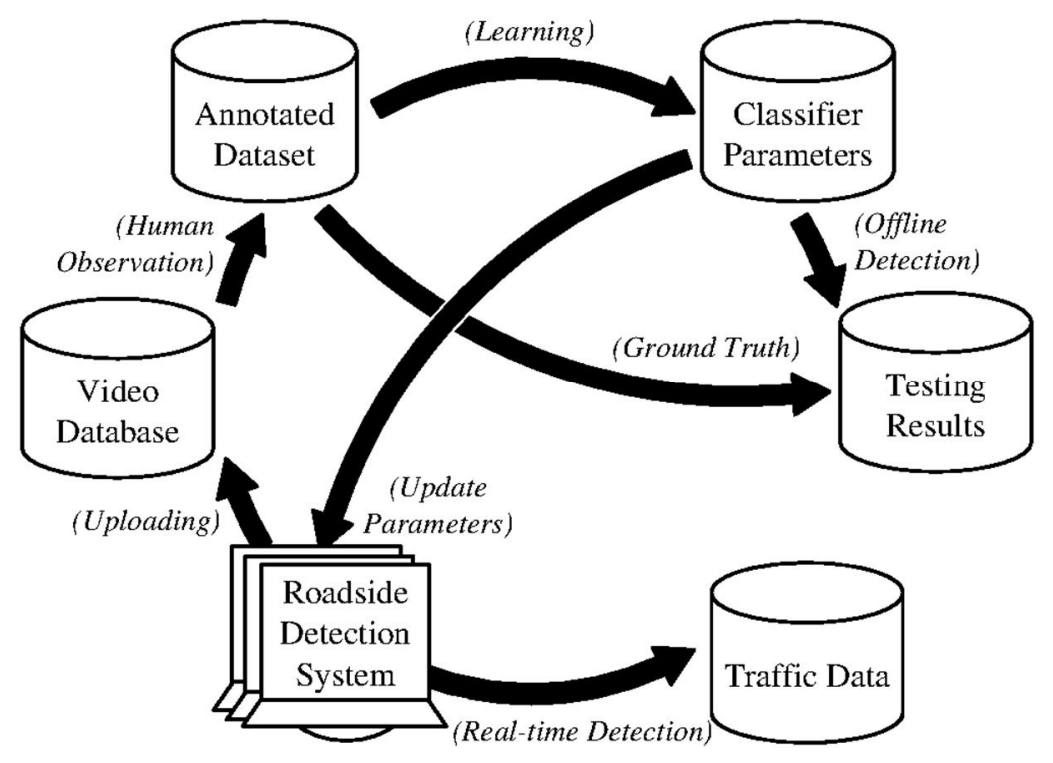

Read Article:

http://arxiv.org/pdf/1510.02055.pdf

City Management Platform Using Big Data from People and Traffic Flows

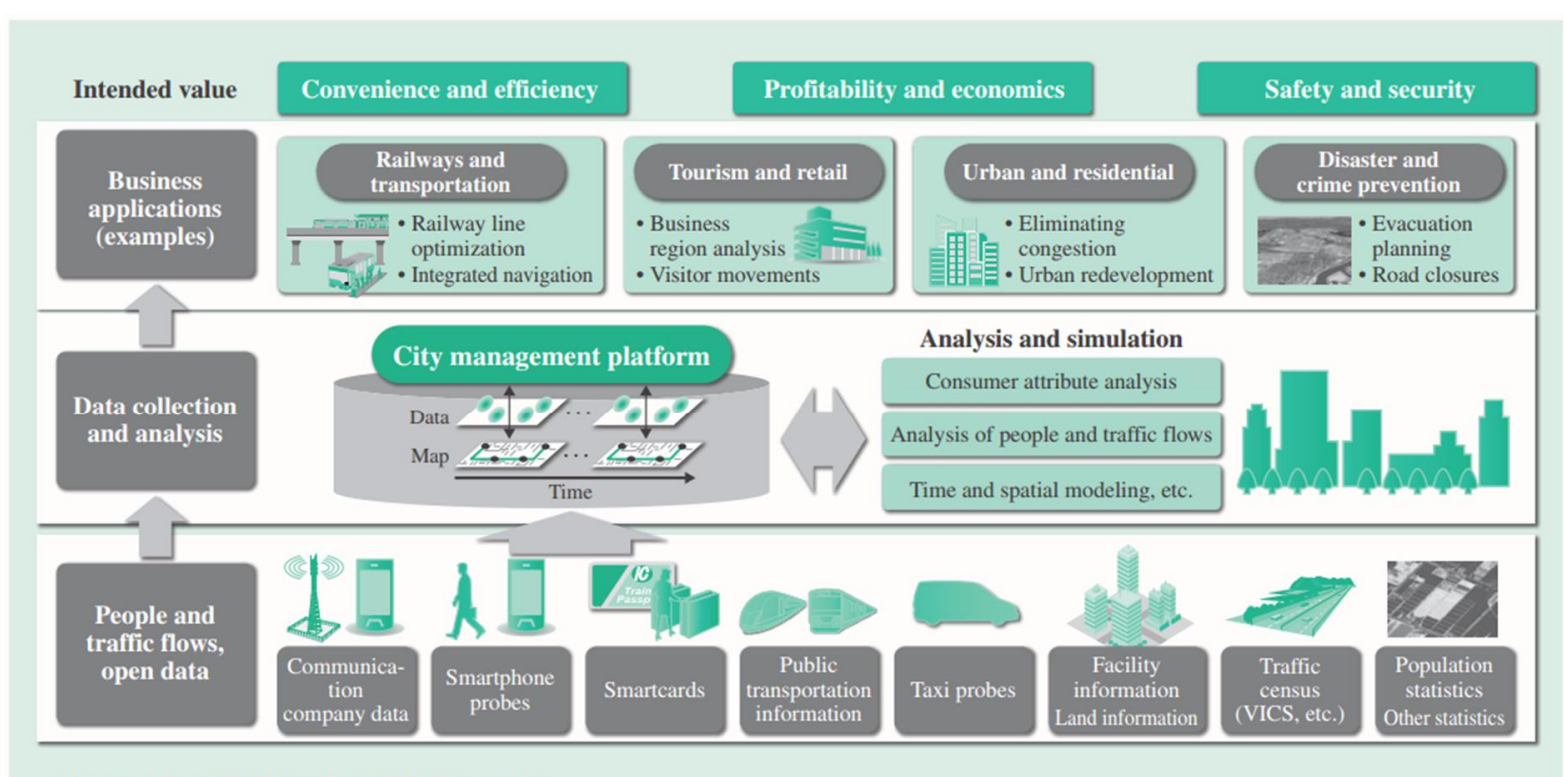

VICS: vehicle information and communication system

Read Article:

http://www.hitachi.com/rev/pdf/2015/r2015_01_108.pdf 


\section{NTT DATA SOLVING TRAFFIC CONGESTION AND ENABLING SMARTER CITIES USING BIG DATA ANALYTICS}

Big-data Simulations for Optimizing Traffic Signal Parameters

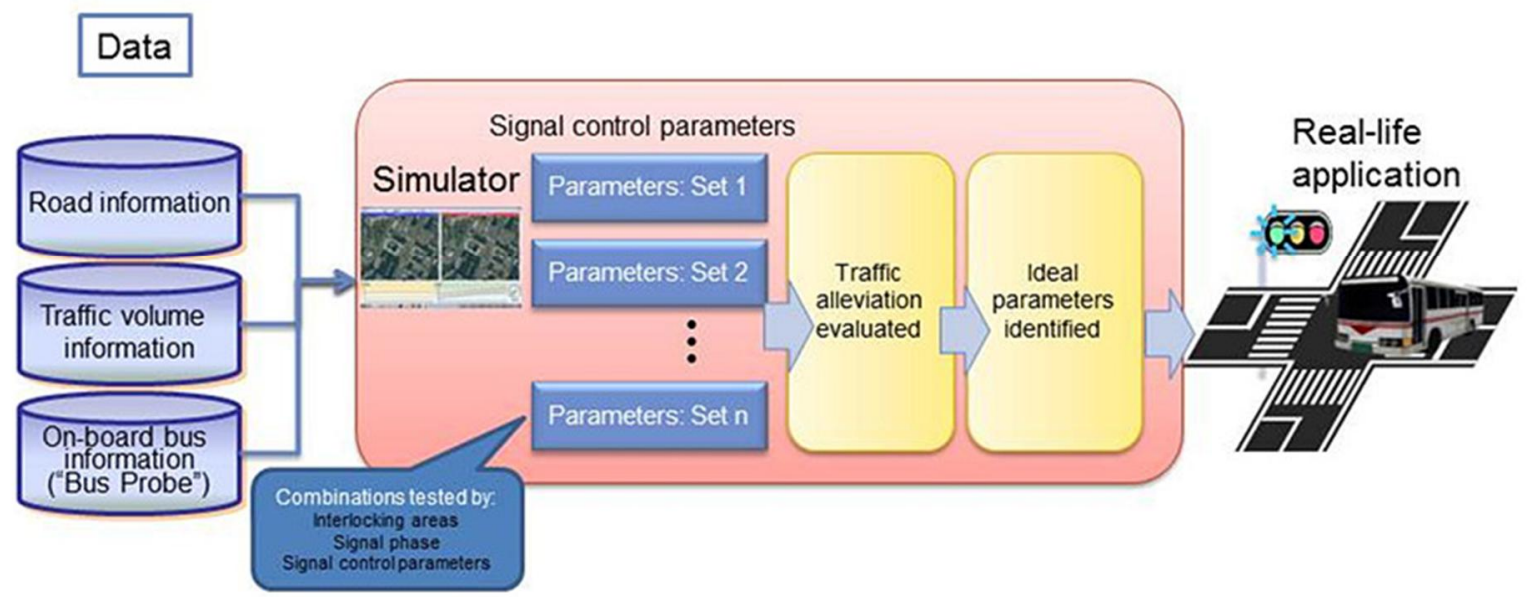

Read Article:

http://www.vcloudnews.com/ntt-data-solving-traffic-congestion-and-enabling-smartercities-using-big-data-analytics/

SMaRTCaR: An integrated smartphone-based platform to support traffic management applications

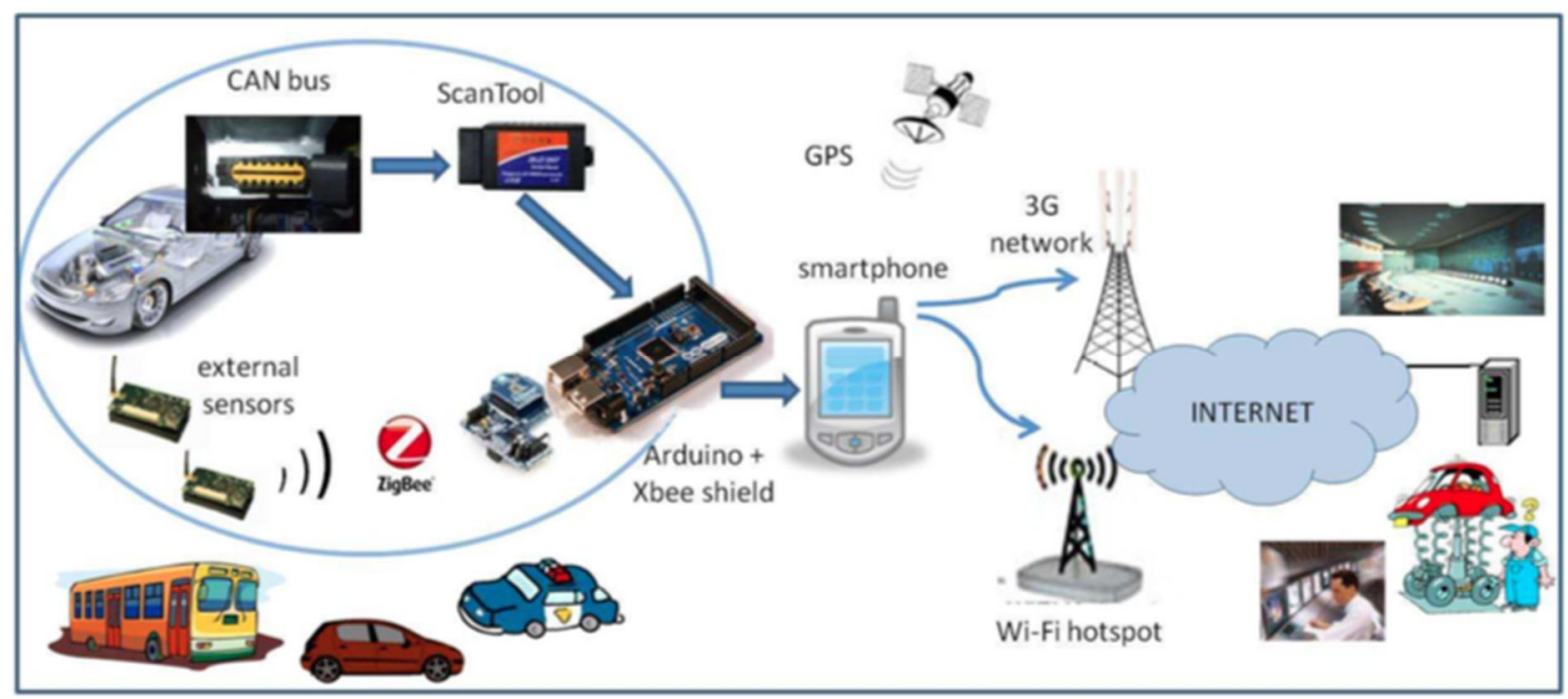

Read Article:

http://ieeexplore.ieee.org/xpl/login.jsp?tp=\&arnumber=6398700\&url=http\%3A\%2F\%2Fie eexplore.ieee.org\%2Fiel5\%2F6384921\%2F6398696\%2F06398700.pdf\%3Farnumber\%3 D6398700 


\section{Intelligent Transportation Systems (ITS) Operations}

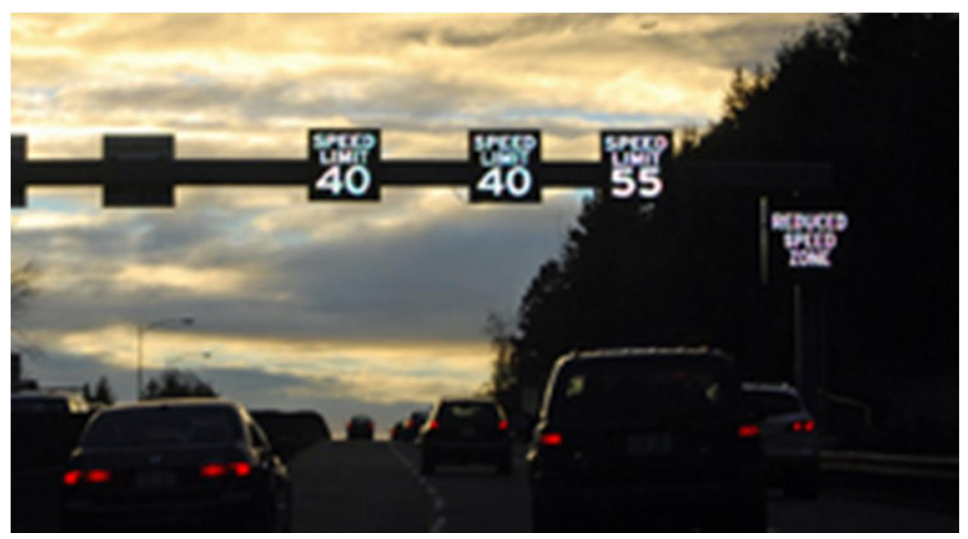

Read Article:

http://www.wsdot.wa.gov/operations/its

\section{Intelligent Transportation Systems}

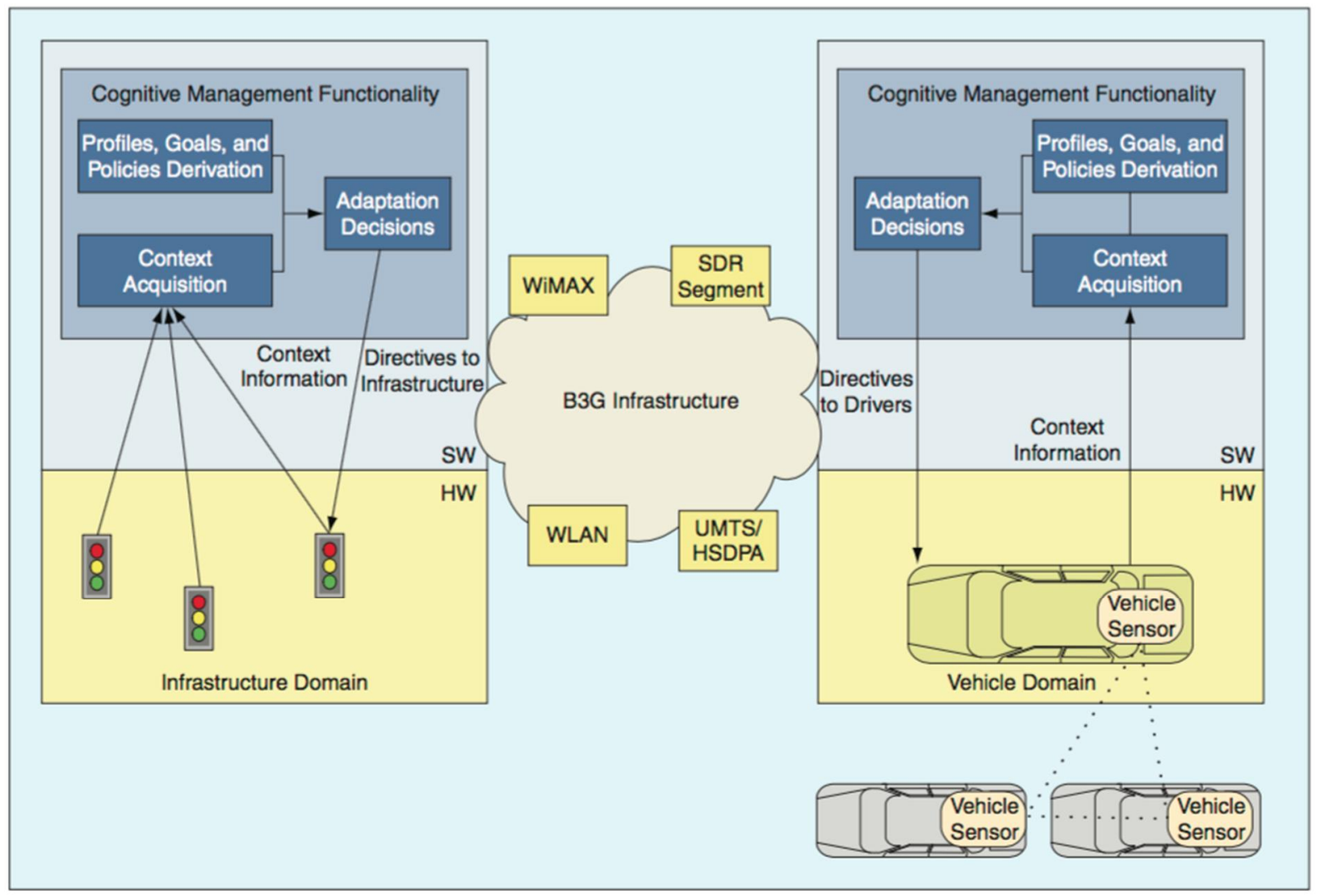

Read Article:

http://ieeexplore.ieee.org/xpl/login.jsp?tp=\&arnumber=5430544\&url=http\%3A\%2F\%2Fie eexplore.ieee.org\%2Fxpls\%2Fabs_all.jsp\%3Farnumber\%3D5430544 
Mobile phone location determination and its impact on intelligent transportation systems

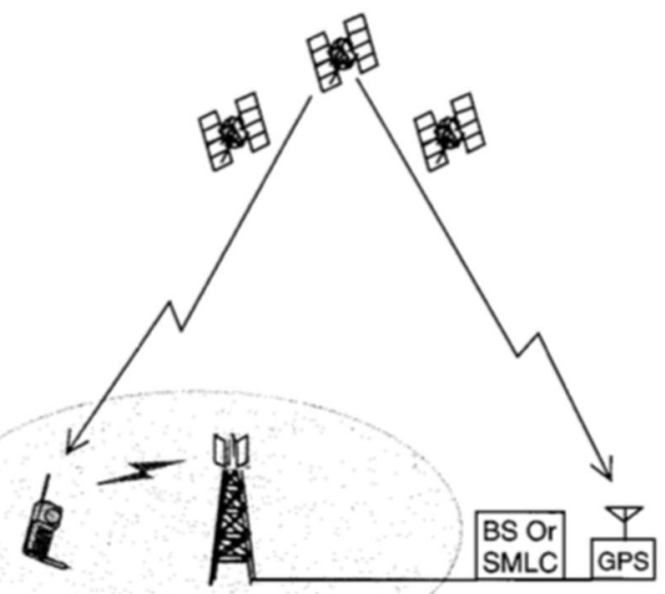

Read Article:

http://ieeexplore.ieee.org/xpl/login.jsp?tp=\&arnumber=869021\&url=http\%3A\%2F\%2Fiee explore.ieee.org\%2Fxpls\%2Fabs_all.jsp\%3Farnumber\%3D869021

SUMMARY OF VEHICLE DETECTION AND SURVEILLANCE TECHNOLOGIES USED IN INTELLIGENT TRANSPORTATION SYSTEMS

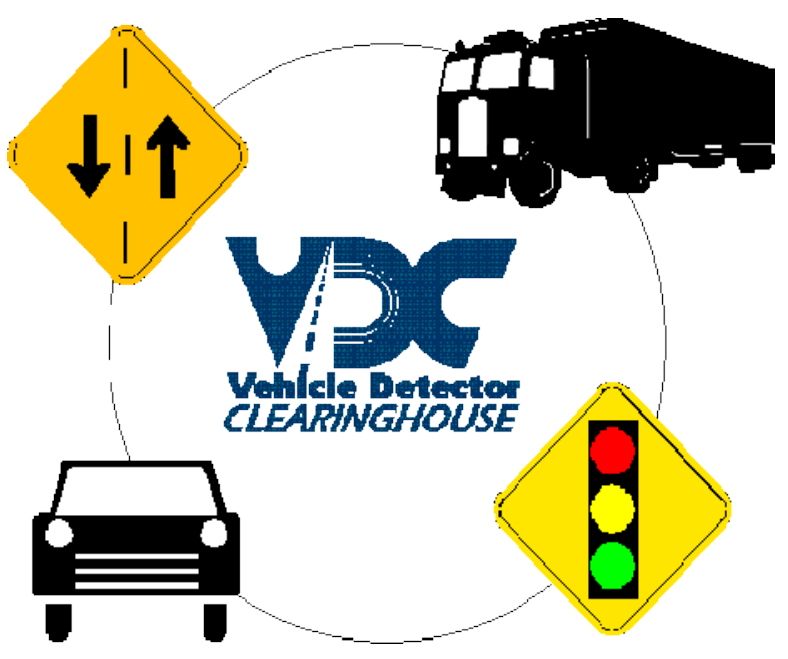

Read Article:

https://trid.trb.org/view.aspx?id=681316 
Toward the development of intelligent transportation systems

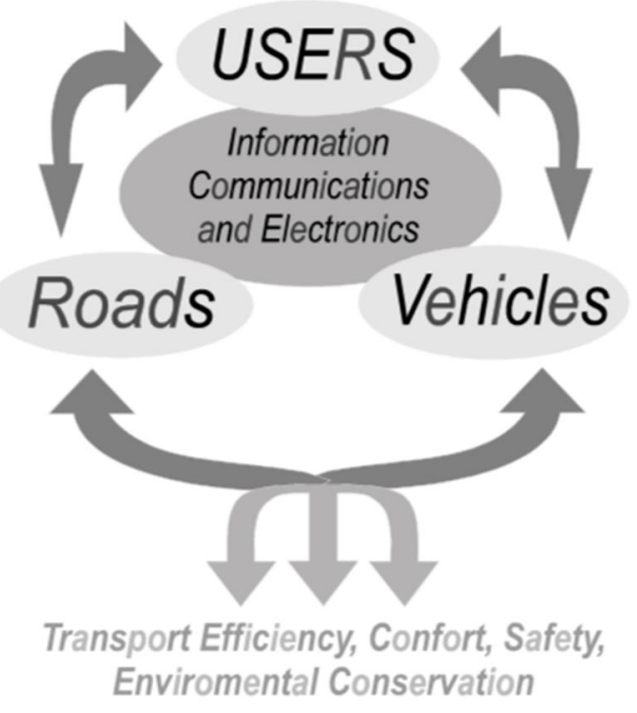

Read Article:

http://www.universelle-automation.de/1984_Columbus.pdf

Parallel Control and Management for Intelligent Transportation Systems: Concepts, Architectures, and Applications

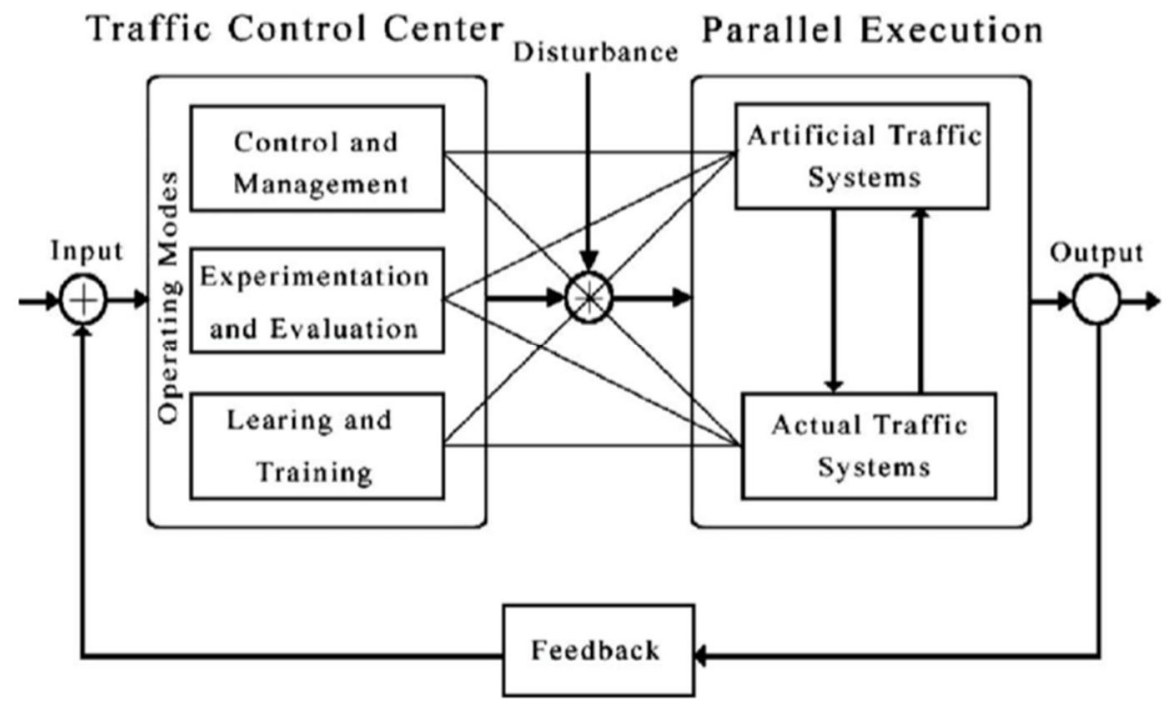

Read Article:

http://ieeexplore.ieee.org/xpl/login.jsp?tp=\&arnumber=5549912\&url=http\%3A\%2F\%2Fie eexplore.ieee.org\%2Fxpls\%2Fabs_all.jsp\%3Farnumber\%3D5549912 
A Survey on Intelligent Transportation Systems

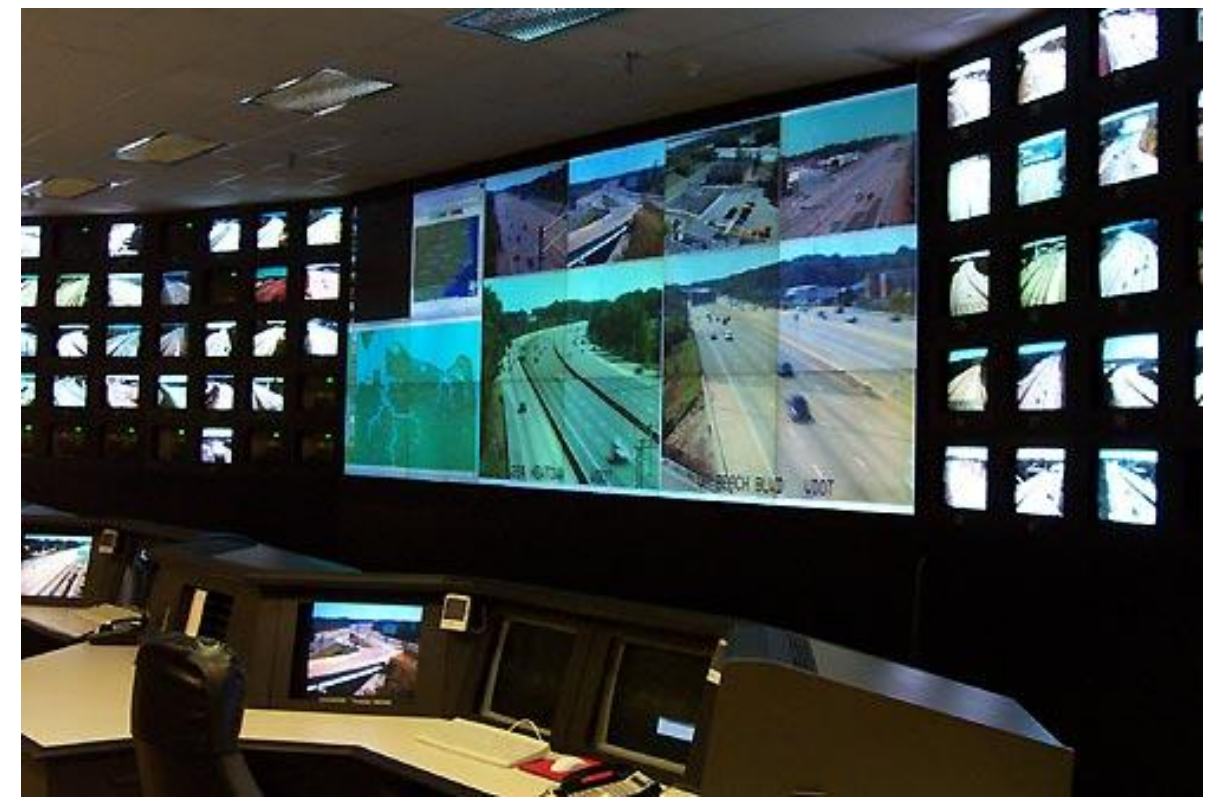

Read Article:

http://www.idosi.org/mejsr/mejsr15(5)13/4.pdf

Performance Analysis of PMIPv6-Based NEtwork MObility for Intelligent Transportation Systems

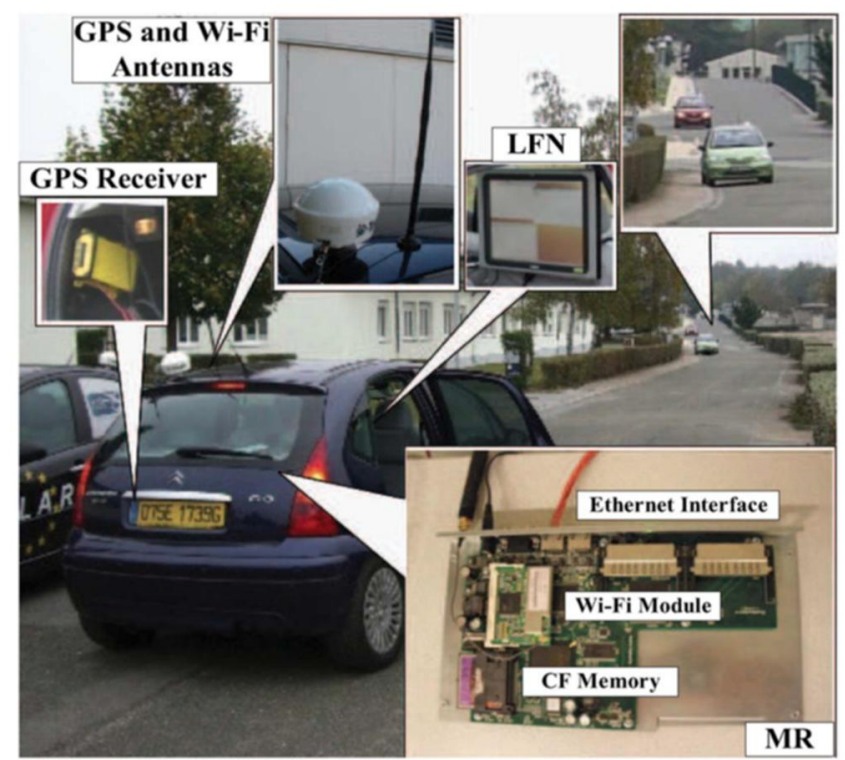

Read Article:

http://ieeexplore.ieee.org/xpl/login.jsp?tp=\&arnumber=5776712\&url=http\%3A\%2F\%2Fie eexplore.ieee.org\%2Fxpls\%2Fabs_all.jsp\%3Farnumber\%3D5776712 
Day-to-day dynamic models for Intelligent Transportation Systems design and appraisal

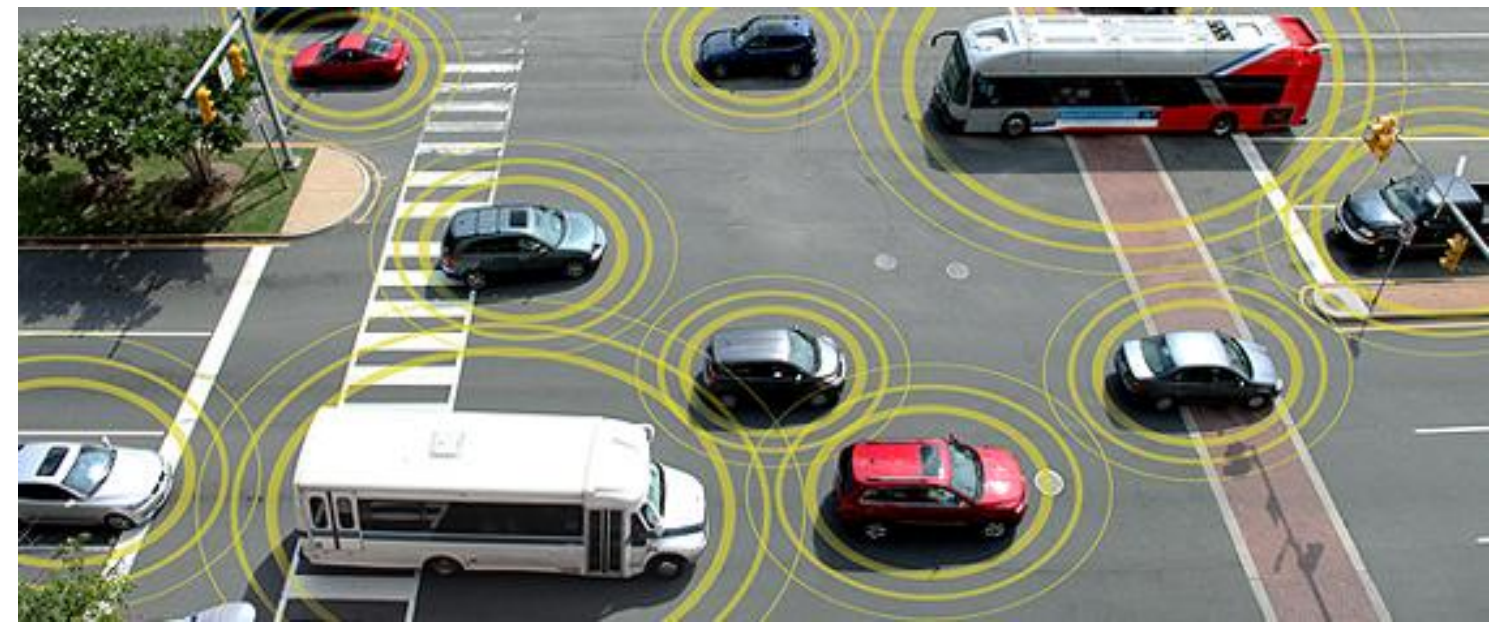

Read Article:

http://www.sciencedirect.com/science/article/pii/S0968090X12000472

Optimization models for assessing the peak capacity utilization of intelligent transportation systems

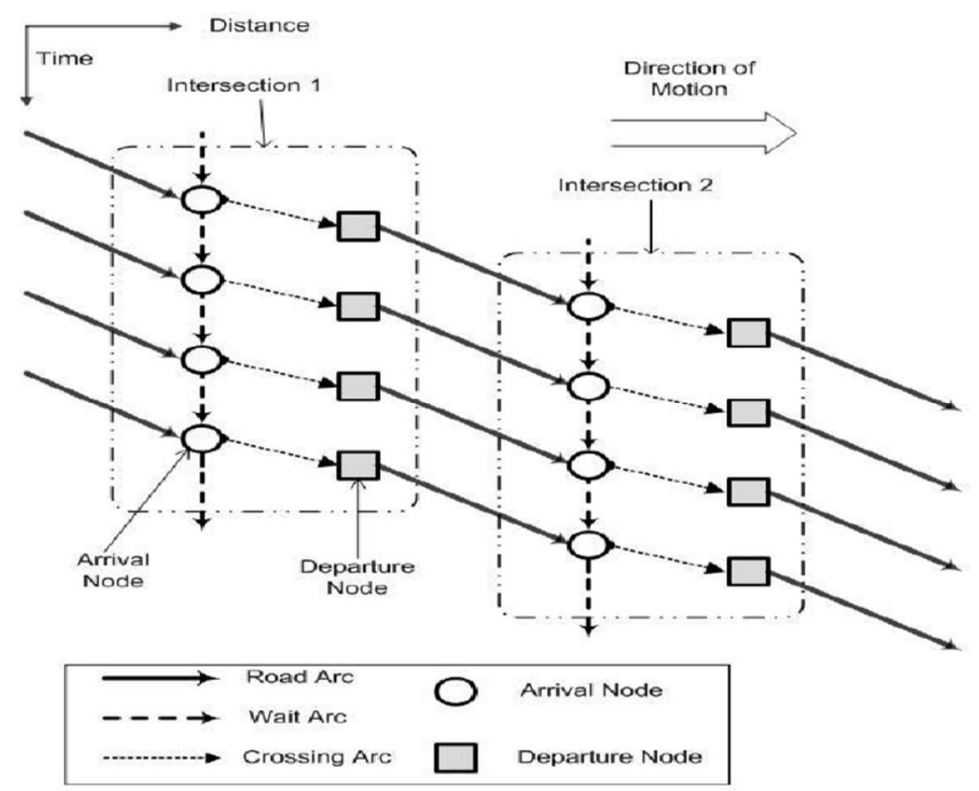

Read Article:

http://www.sciencedirect.com/science/article/pii/S0377221711006643 


\section{CrowdITS: Crowdsourcing in intelligent transportation} systems

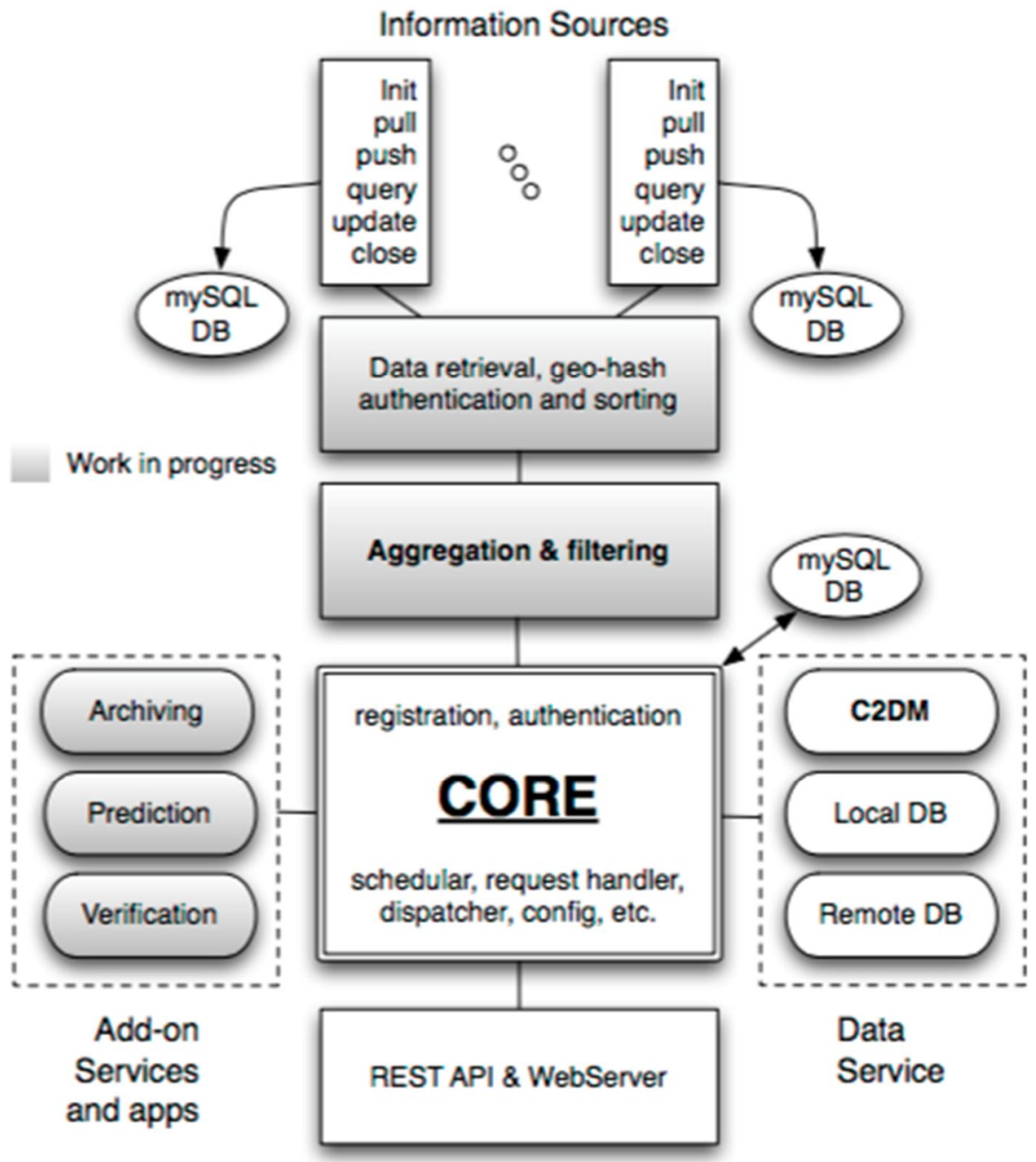

Read Article:

http://ieeexplore.ieee.org/xpl/login.jsp?tp=\&arnumber=6214379\&url=http\%3A\%2F\%2Fie eexplore.ieee.org\%2Fxpls\%2Fabs_all.jsp\%3Farnumber\%3D6214379

Intelligent transportation systems for smart cities: a progress review

Read Article:

http://link.springer.com/article/10.1007/s11432-012-4725-1 
A review on vision-based pedestrian detection in intelligent transportation systems

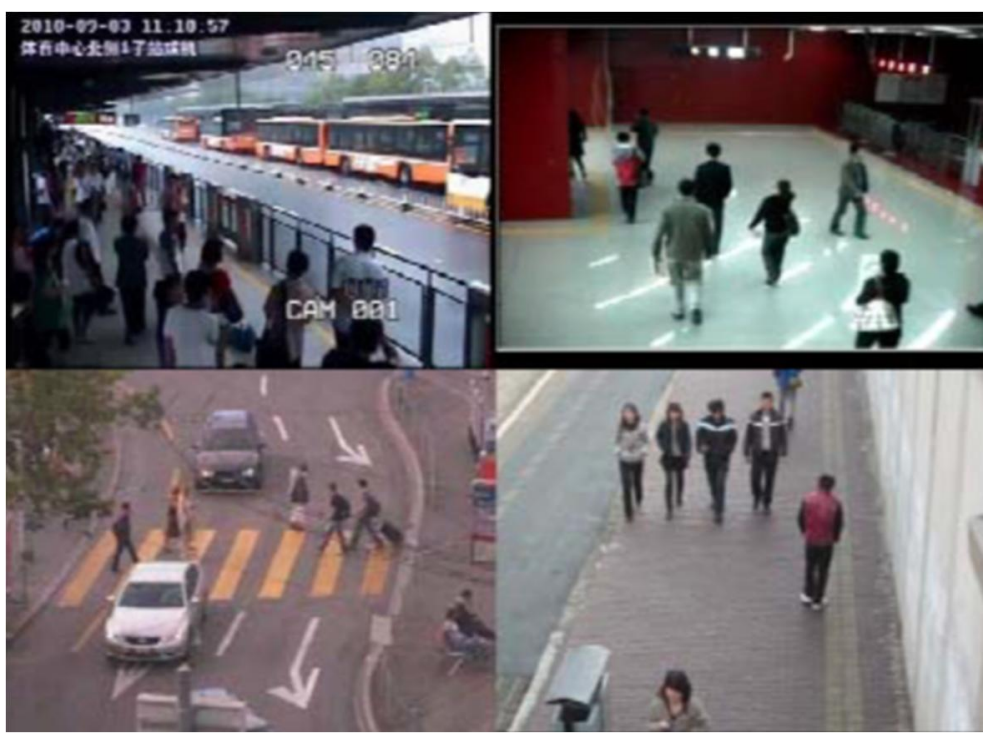

Read Article:

http://ieeexplore.ieee.org/xpl/login.jsp?tp=\&arnumber=6204951\&url=http\%3A\%2F\%2Fie eexplore.ieee.org\%2Fxpls\%2Fabs_all.jsp\%3Farnumber\%3D6204951

Meeting privacy challenges while advancing intelligent transportation systems

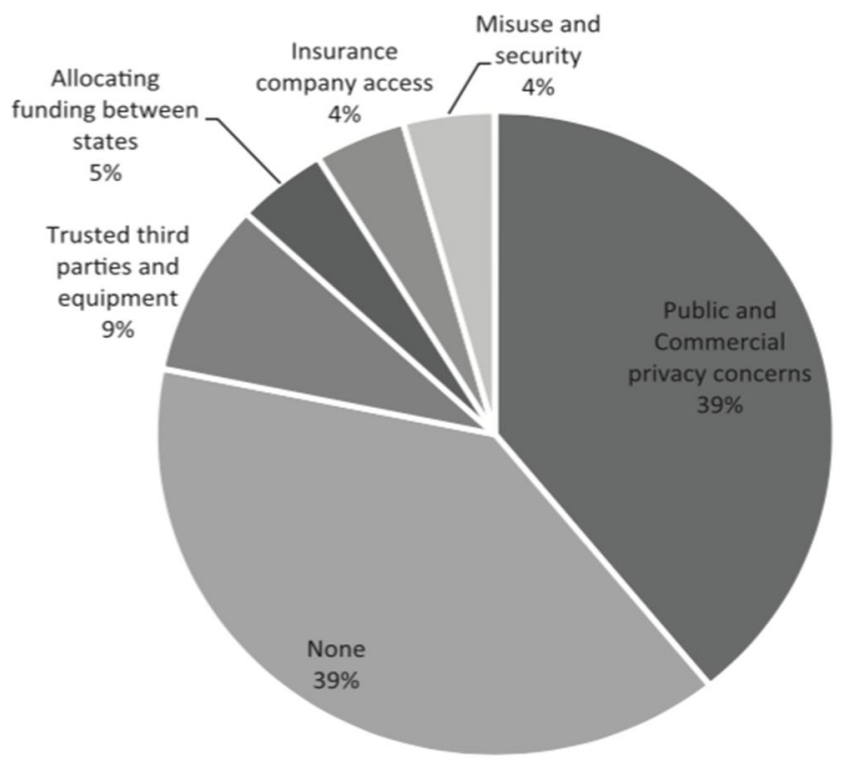

Read Article:

http://www.sciencedirect.com/science/article/pii/S0968090X12000526 
Context-Aware Driver Behavior Detection System in Intelligent Transportation Systems

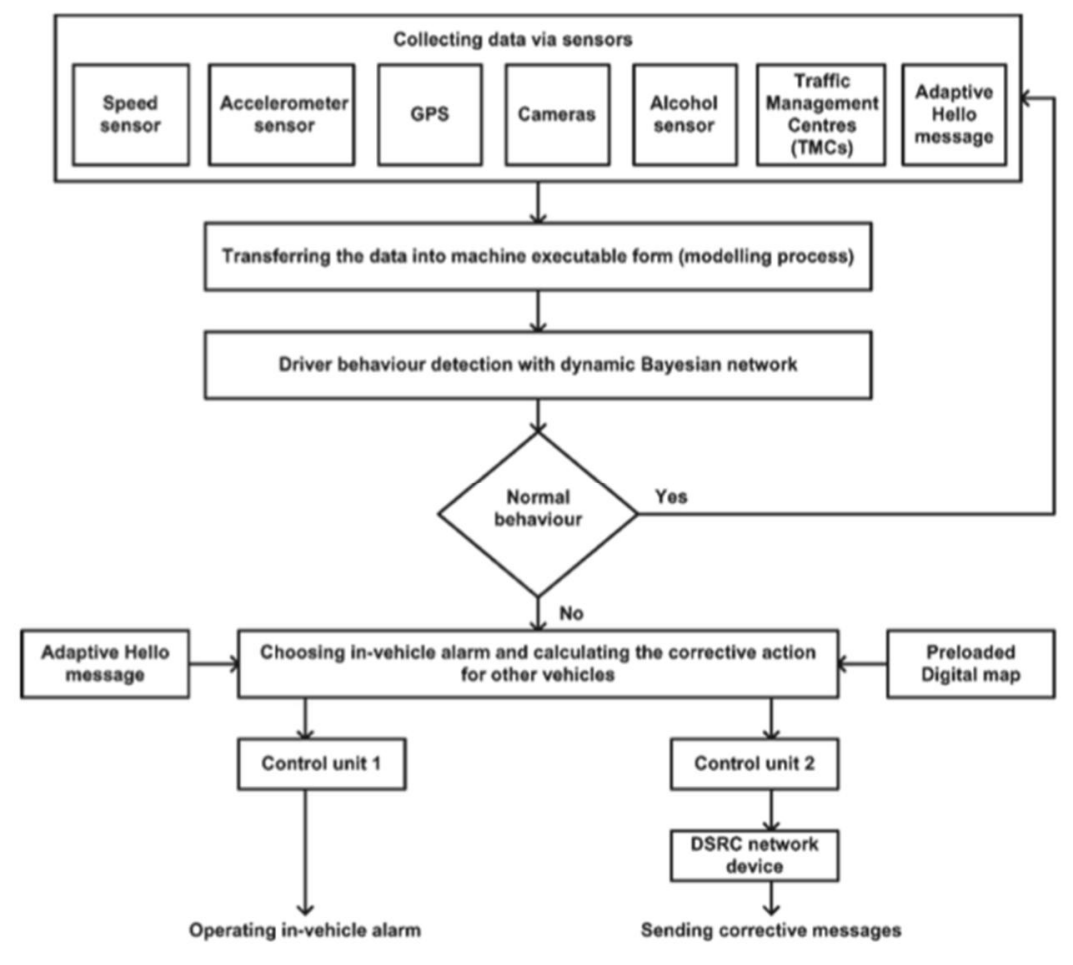

Read Article:

http://ieeexplore.ieee.org/xpl/login.jsp?tp=\&arnumber=6516983\&url=http\%3A\%2F\%2Fie eexplore.ieee.org\%2Fxpls\%2Fabs_all.jsp\%3Farnumber\%3D6516983

\section{Research and Development of Intelligent Transportation Systems}
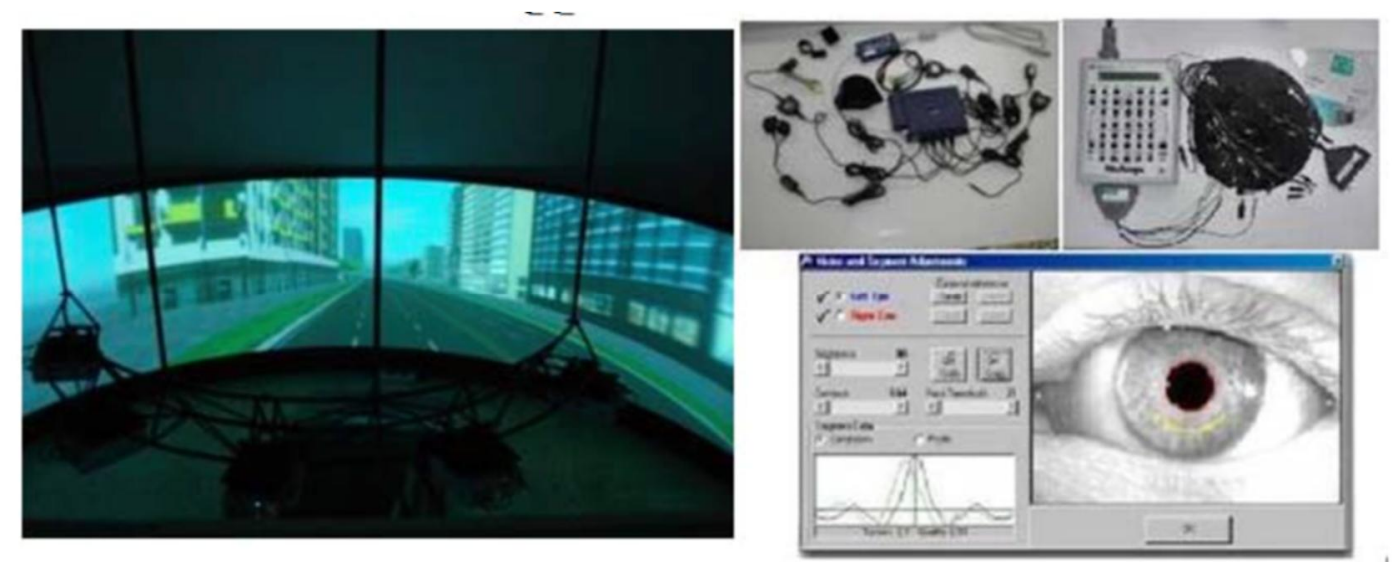

Read Article:

http://ieeexplore.ieee.org/xpl/login.jsp?tp=\&arnumber=6385299\&url=http\%3A\%2F\%2Fie eexplore.ieee.org\%2Fxpls\%2Fabs_all.jsp\%3Farnumber\%3D6385299 
A multi-agent approach to Intelligent Transportation Systems modeling with combinatorial auctions

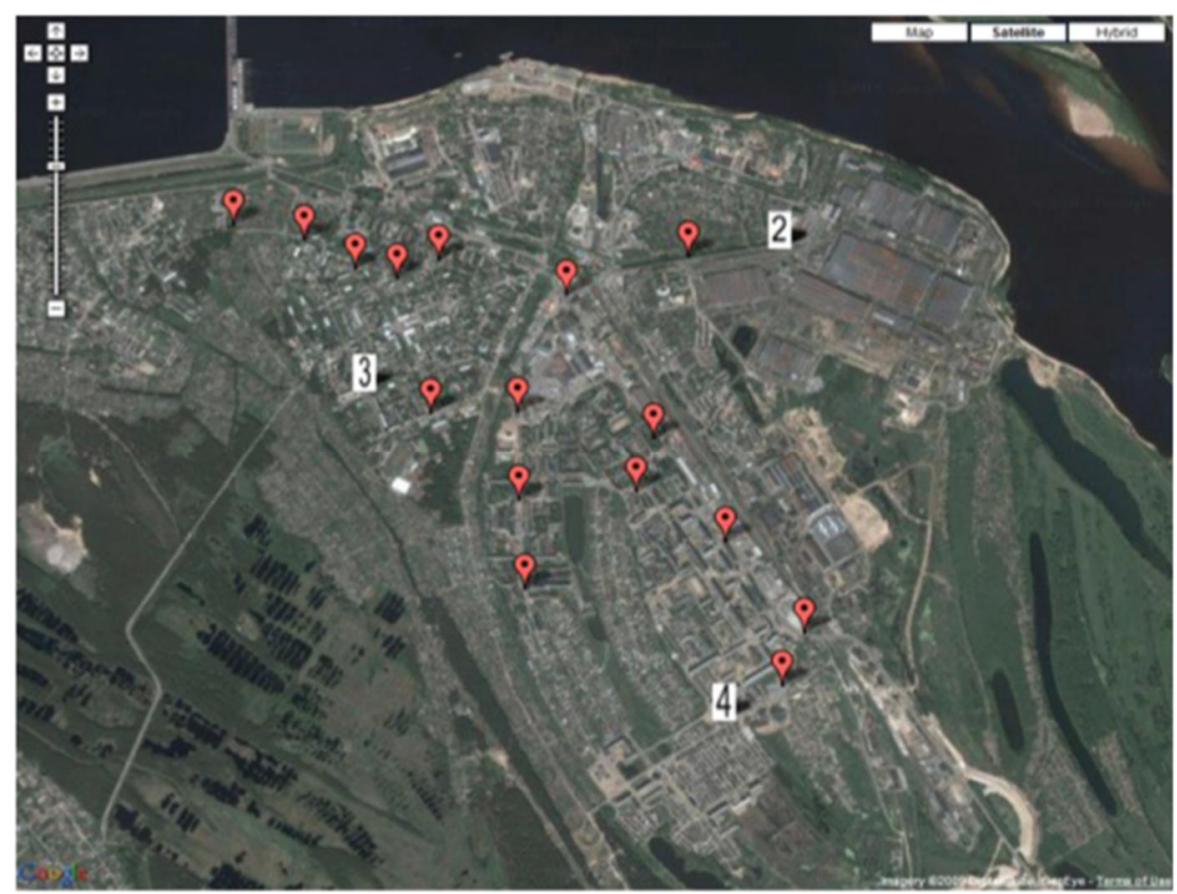

Read Article:

http://www.sciencedirect.com/science/article/pii/S0957417414002954

Sensor and network technology for intelligent transportation systems
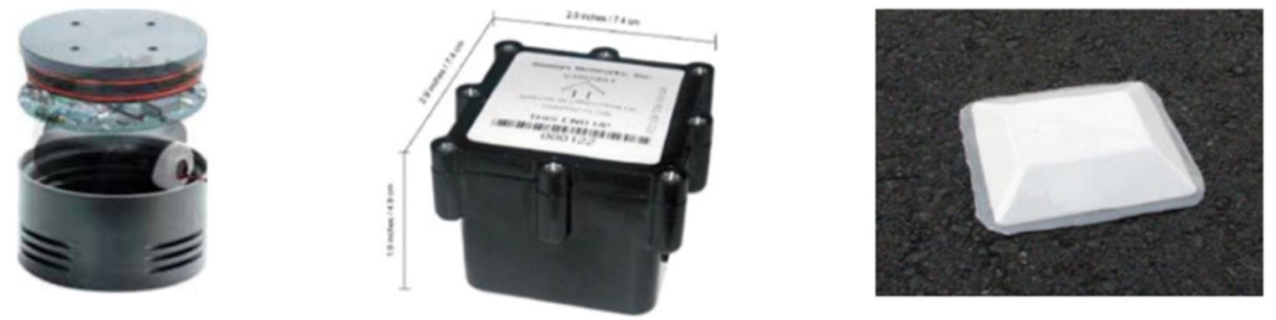

Read Article:

http://ieeexplore. ieee.org/xpl/login.jsp?tp=\&arnumber=6220735\&url=http\%3A\%2F\%2Fie eexplore.ieee.org\%2Fxpls\%2Fabs_all.jsp\%3Farnumber\%3D6220735 
Recent trends in intelligent transportation systems: a review

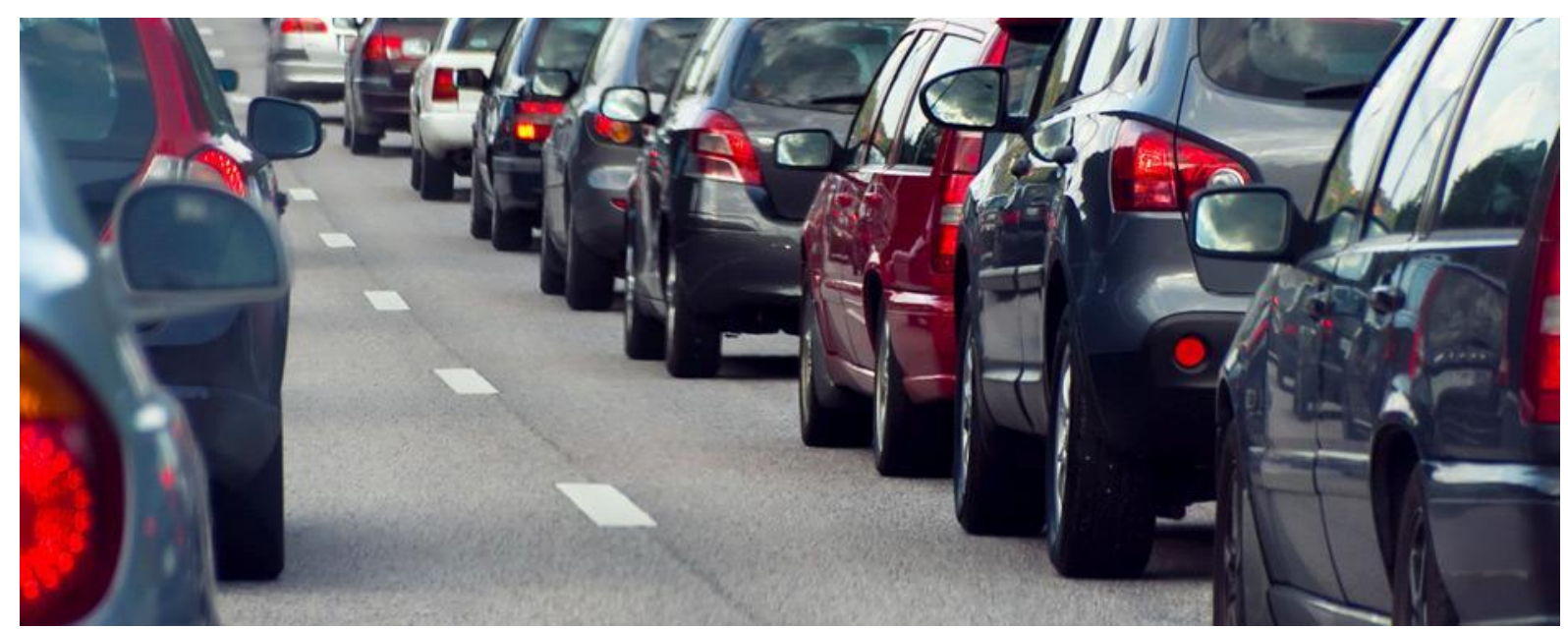

Read Article:

http://www.scielo.br/scielo.php?pid=S2238-

10312015000200030\&script=sci_arttext\&tIng=es

Intelligent Transportation Systems

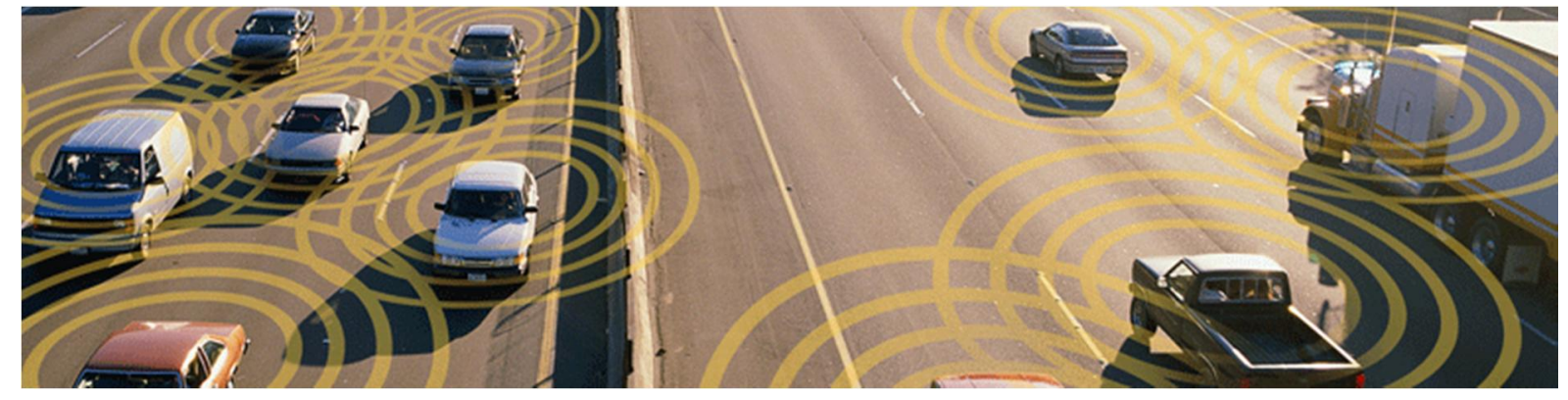

Read Article:

https://www.transportation.alberta.ca/606.htm

A Comparative Study of Three Multivariate Short-Term Freeway Traffic Flow Forecasting Methods With Missing Data

Read Article:

http://www.tandfonline.com/doi/pdf/10.1080/15472450.2016.1147813 
Build intelligent transportation systems with the Traffic Management Data Dictionary standard

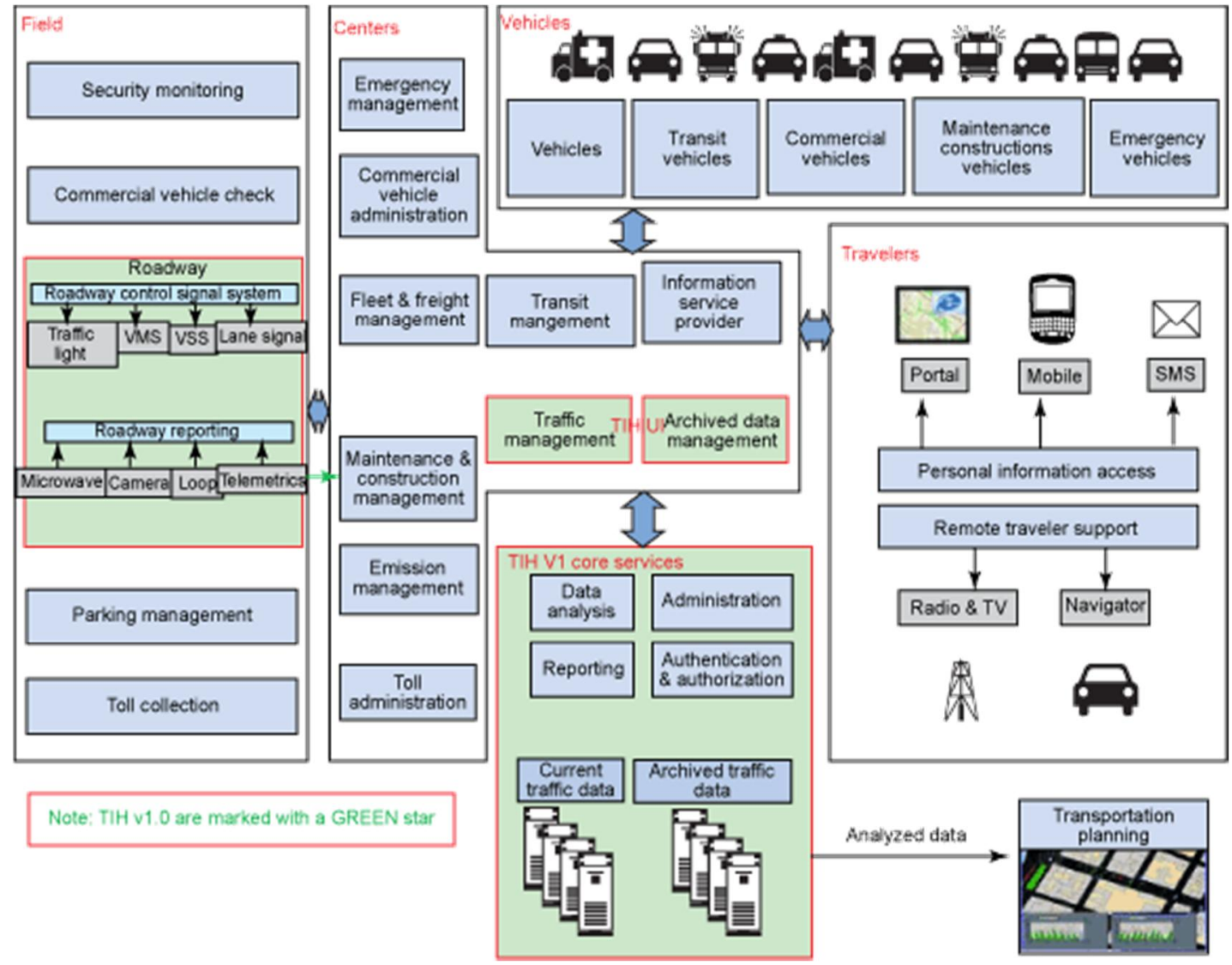

Read Article:

http://www.ibm.com/developerworks/industry/library/ind-tmdd/ind-tmdd-pdf.pdf

\section{Practical Camera Calibration From Moving Objects for} Traffic Scene Surveillance

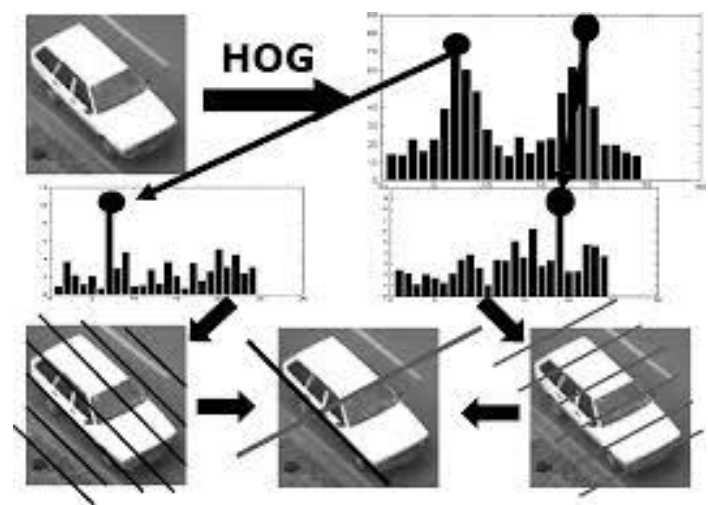

Read Article:

http://ieeexplore.ieee.org/stamp/stamp.jsp?arnumber=6252024 


\section{Advanced Urban Traffic Management System}

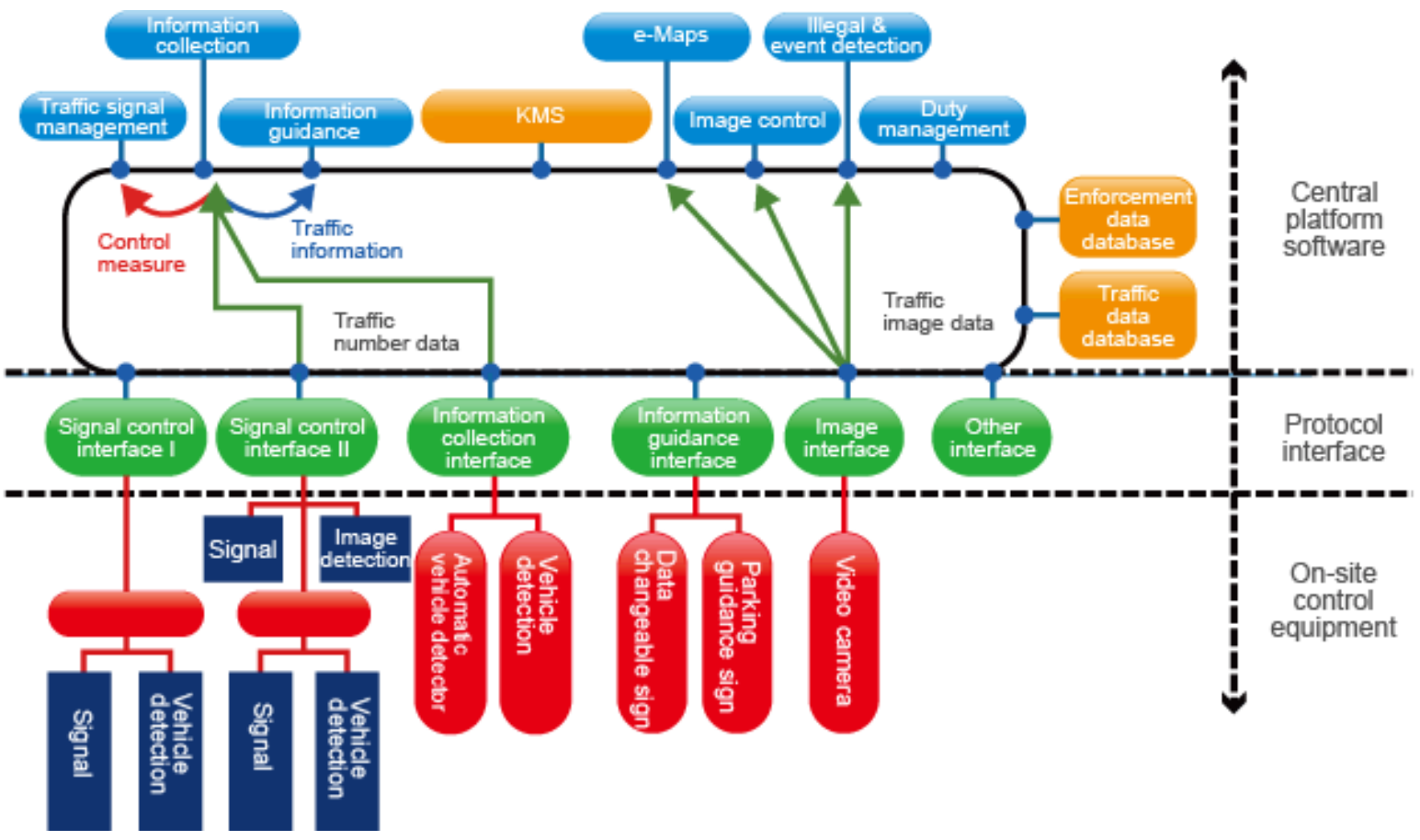

Read Article:

http://www.iisigroup.com/en/products/tra-autms.html

Sustainable Transportation Ecosystem Addressing sustainability from an integrated systems perspective

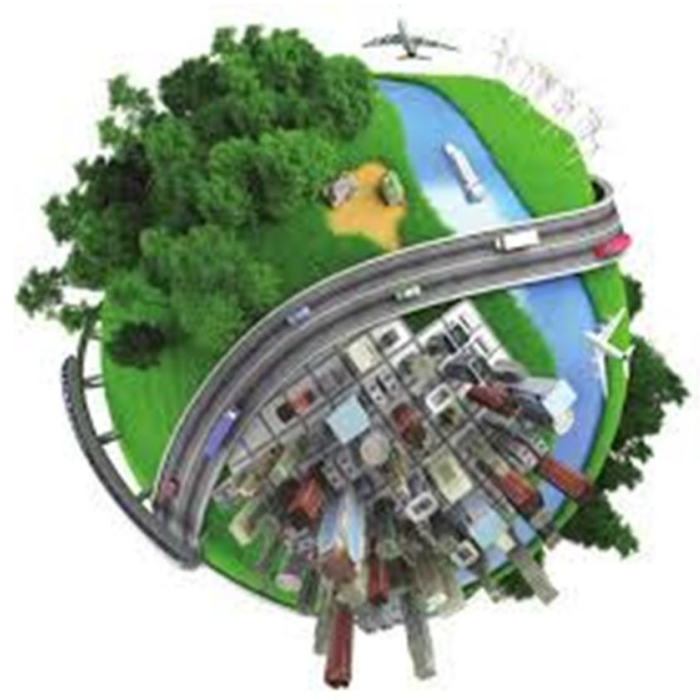

Read Article:

https://www.atkearney.com/documents/10192/346302/STEReport.pdf/71 caa02e-63994cbf-9e32-d2a6dc58dd1d 


\section{Digital-Age Transportation. The Future of Urban Mobility}

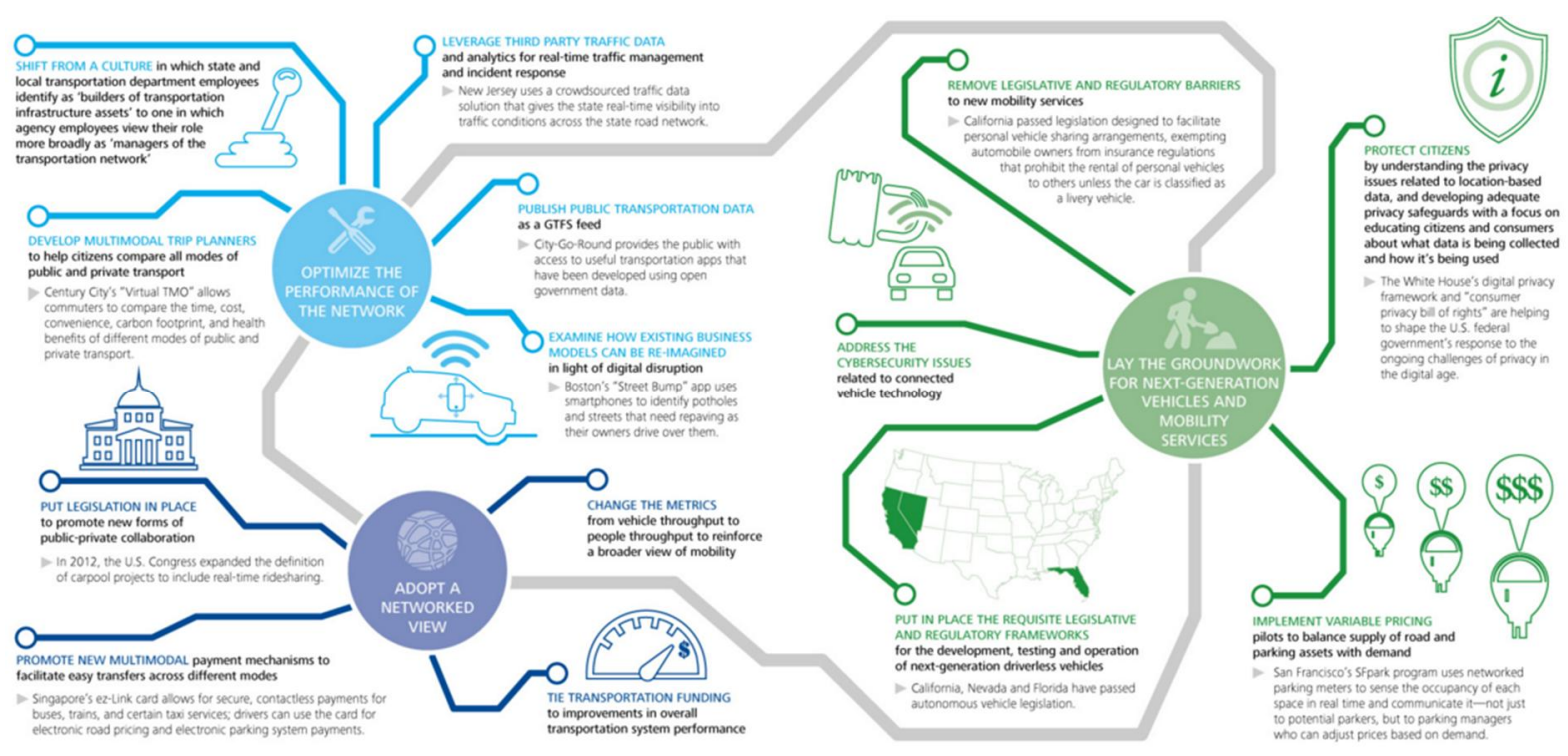

Read Article:

http://dupress.com/articles/digital-age-transportation/

\section{Transport Ecosystem}

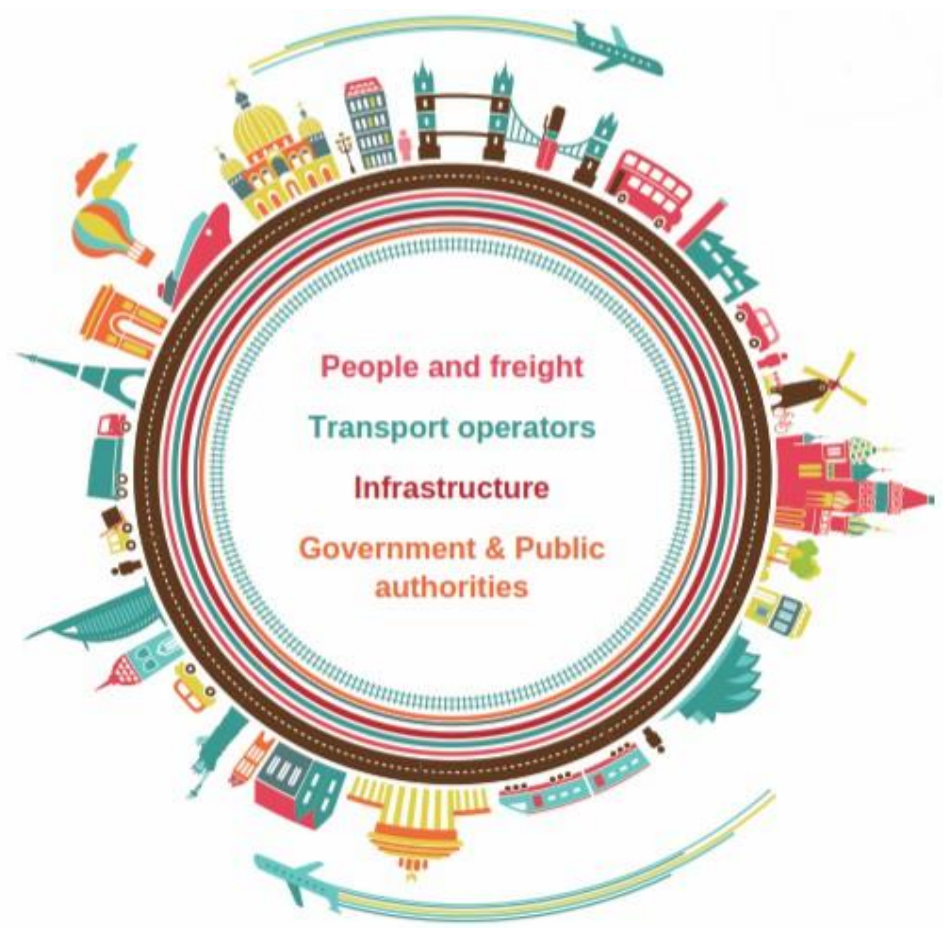

Read Article:

https://home.kpmg.com/xx/en/home/insights/2015/09/transport-ecosystem.html 


\section{The future of mobility. How transportation technology and social trends are creating a new business ecosystem}

\begin{tabular}{|l|l|}
\hline \multicolumn{1}{|c|}{ Insider view } \\
$\begin{array}{l}\text { The industry will evolve } \\
\text { naturally and incremen- } \\
\text { tally toward a future } \\
\text { mobility system that } \\
\text { retains its roots in what } \\
\text { exists today }\end{array}$ \\
$\begin{array}{l}\text { The key players, major } \\
\text { assets, and overall } \\
\text { structure of the current } \\
\text { ecosystem can remain } \\
\text { intact while change } \\
\text { progresses in an orderly, } \\
\text { linear fashion }\end{array}$ \\
$\begin{array}{l}\text { The incumbent mindset } \\
\text { appears dually focused } \\
\text { on sustaining the current } \\
\text { model while testing } \\
\text { change in small ways }\end{array}$
\end{tabular}

Read Article:

http://dupress.com/articles/future-of-mobility-transportation-technology/

Where is public transportation going? Insights from the high performers

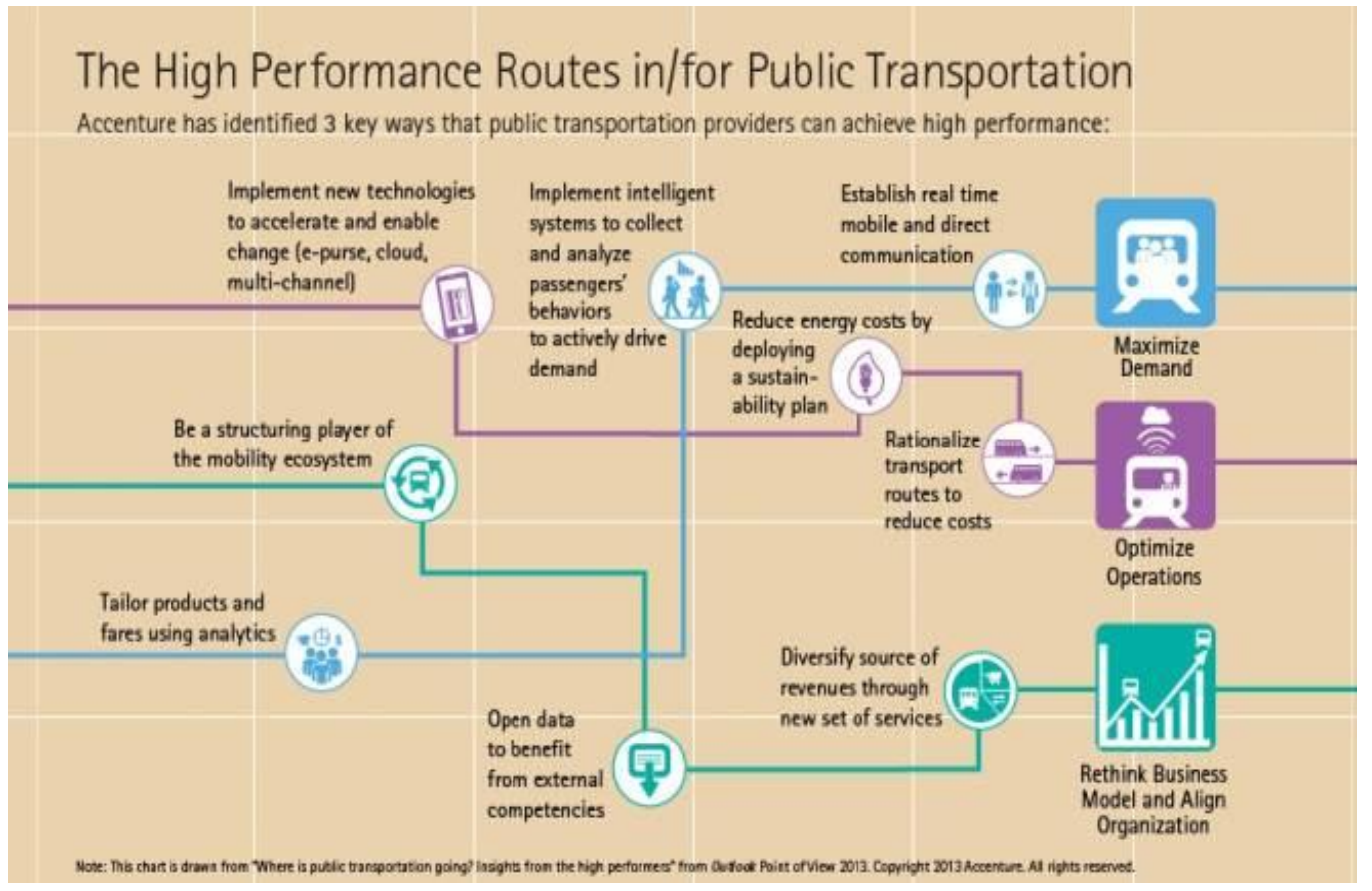

Read Article:

https://www.accenture.com/us-en/insight-outlook-where-is-public-transportation-goinginsight-from-high-performers 
Why the Transportation Industry is Getting on Board with Big Data \& Hadoop

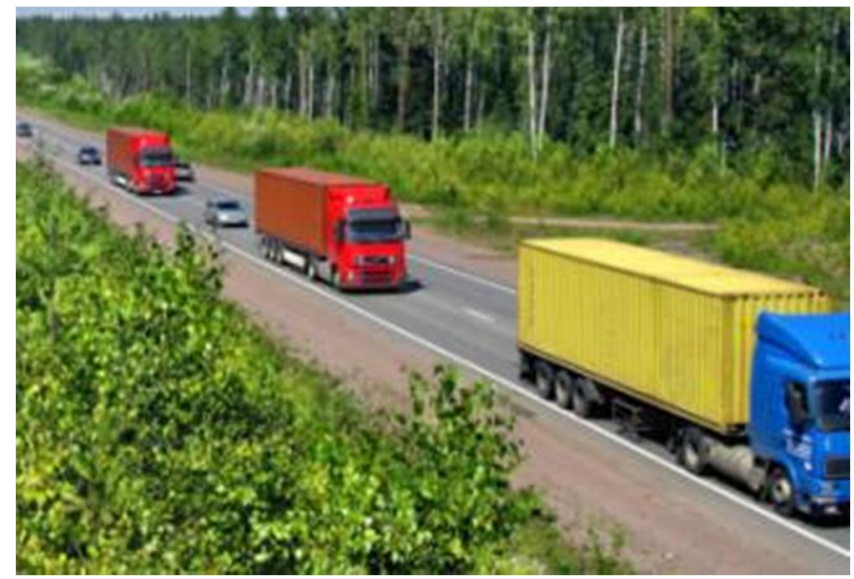

Read Article:

https://www.mapr.com/blog/why-transportation-industry-getting-board-big-data-hadoop

Big Data's Implications for Transportation Operations. An Exploration

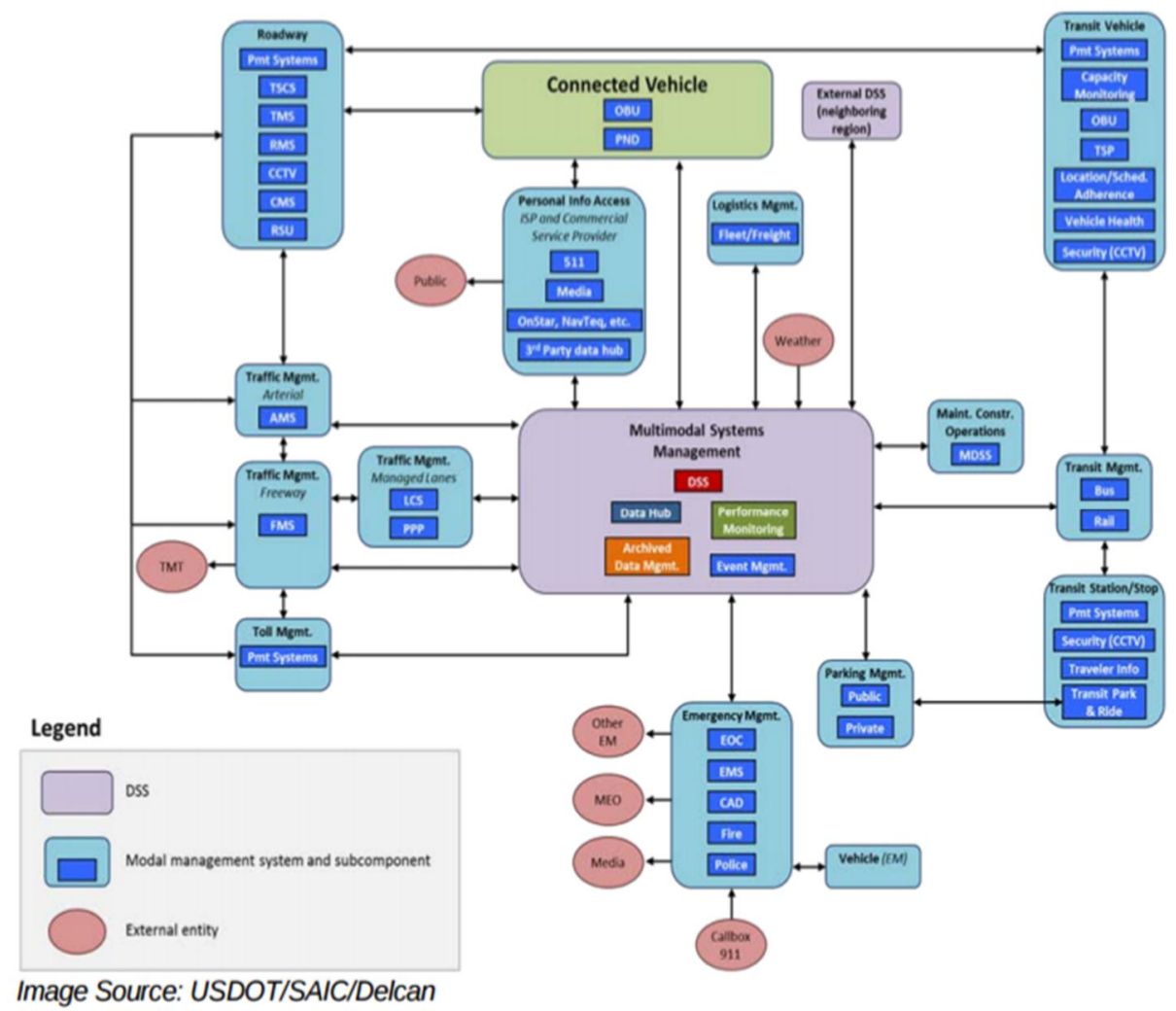

Read Article:

http://ntl.bts.gov/lib/55000/55000/55002/Big_Data_Implications_FHWA-JPO-14-157.pdf 
Maximizing Returns through Advanced Analytics in Transportation

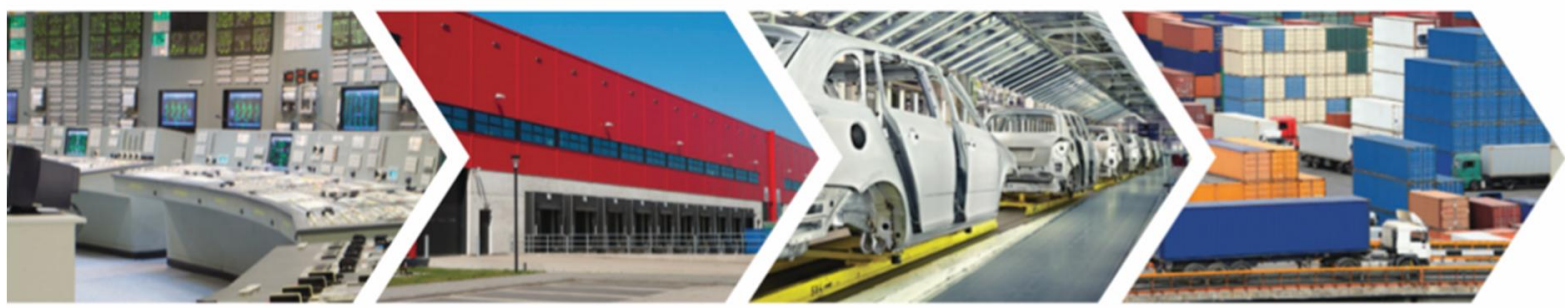

Read Article:

https://quartetfs.com/images/pdf/white-papers/Quartet_FS_White_Paper_-

Transportation.pdf

Transport Intermodality: Integrated Transport for the 21st Century

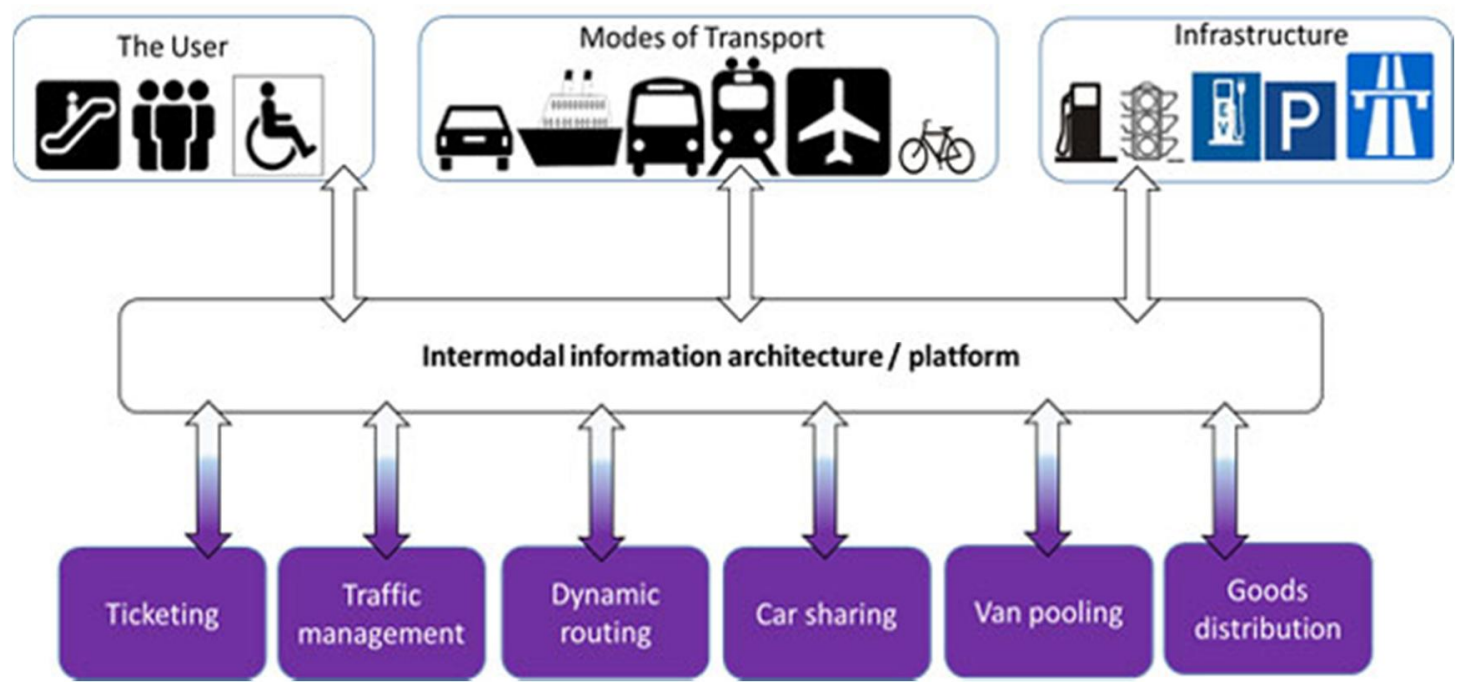

Read Article:

http://www.eurotransportmagazine.com/17415/transport-extra/transport-intermodalityintegrated-transport-for-the-21st-century/ 
Intelligent transport management system for urban traffic hubs based on an integration of multiple technologies

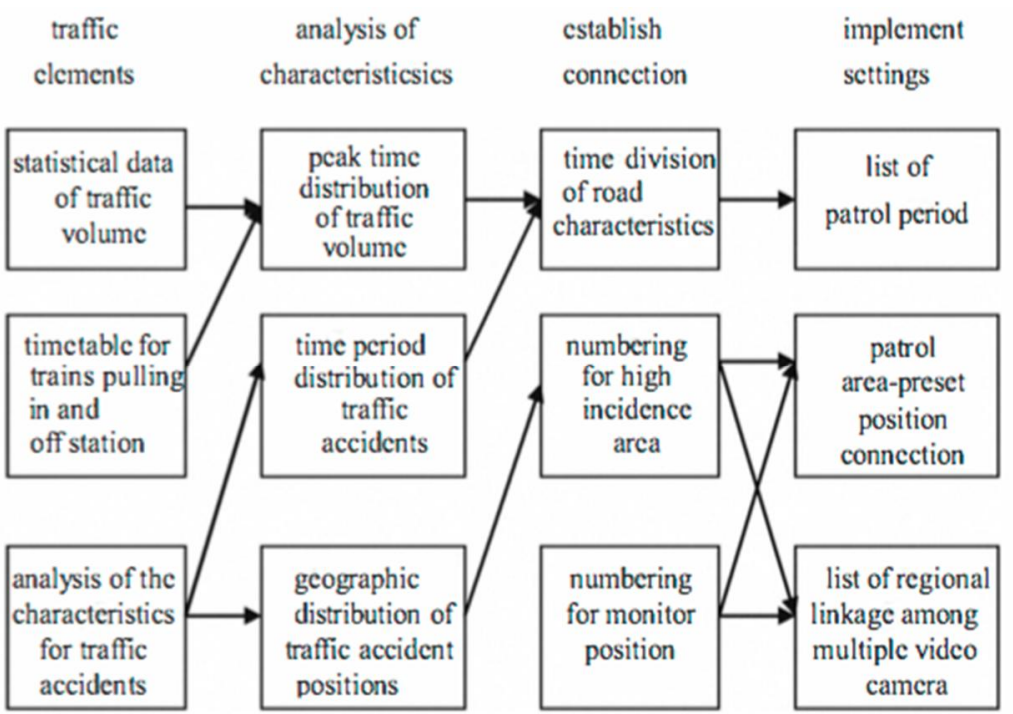

Read Article:

http://ieeexplore. ieee.org/xpl/login.jsp?tp=\&arnumber=5646001\&url=http\%3A\%2F\%2Fie eexplore.ieee.org\%2Fxpls\%2Fabs_all.jsp\%3Farnumber\%3D5646001

\section{Assessing the Potential Impacts of Connected Vehicles: Mobility, Environmental, and Safety Perspectives}

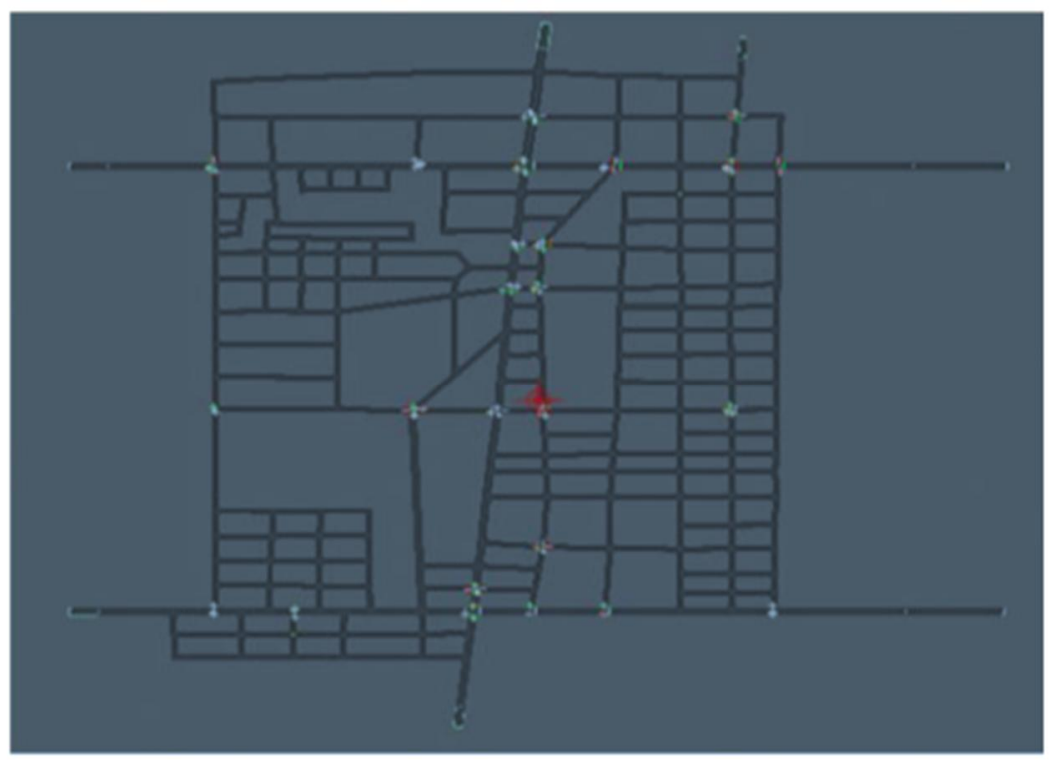

Read Article: 
Comparison of In-Vehicle Auditory Public Traffic Information With Roadside Dynamic Message Signs

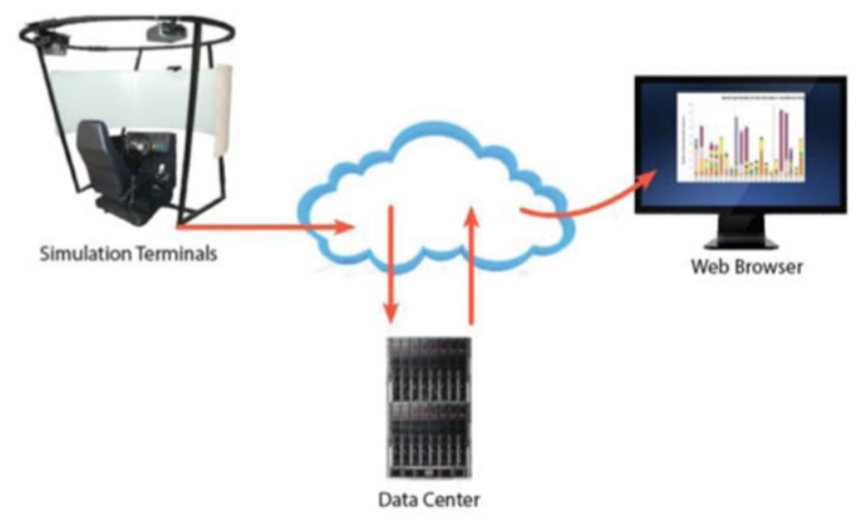

Read Article:

http://www.tandfonline.com/doi/pdf/10.1080/15472450.2015.1062729

\section{Parking Spaces Repurchase Strategy Design via Simulation Optimization}

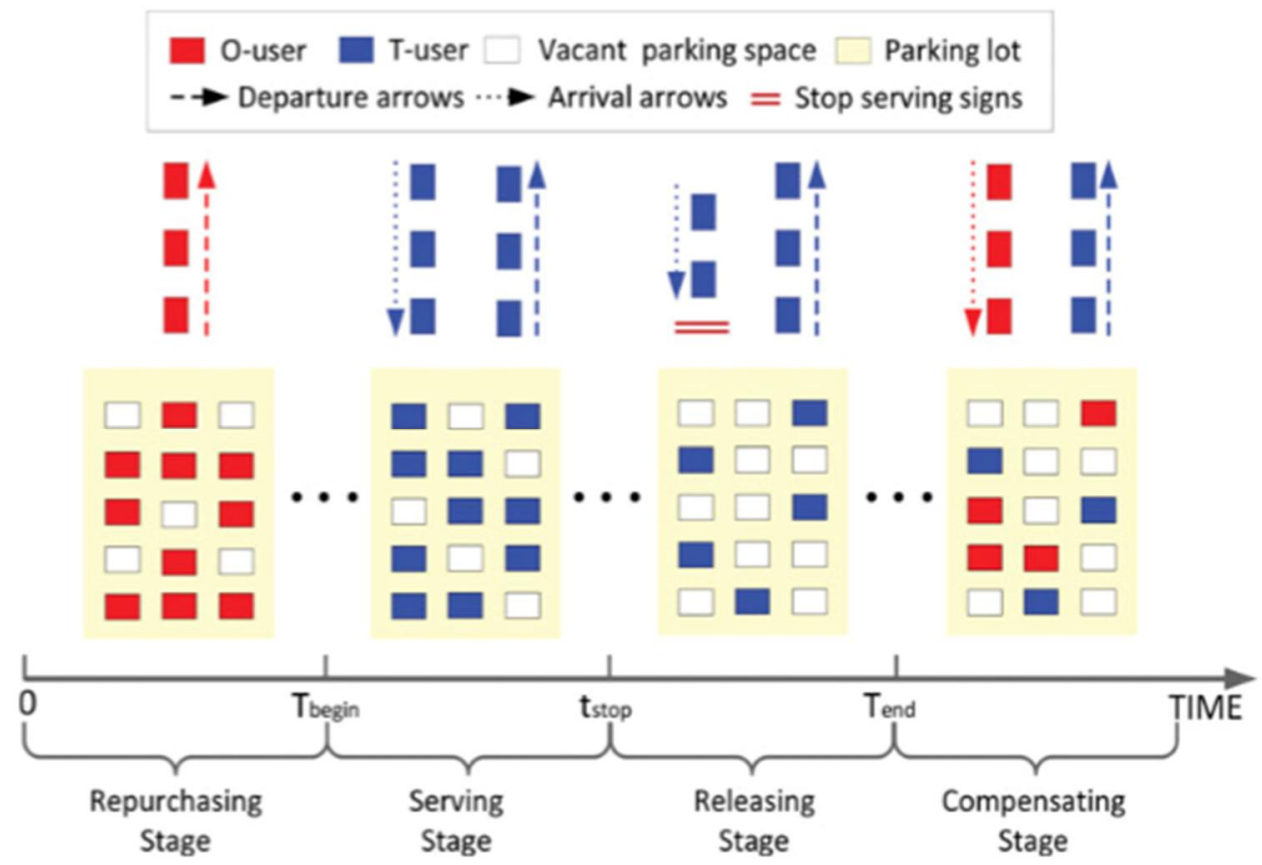

Read Article:

http://www.tandfonline.com/doi/abs/10.1080/15472450.2015.1063424 
Urban Traffic Flow Prediction Using a Spatio-Temporal Random Effects Model

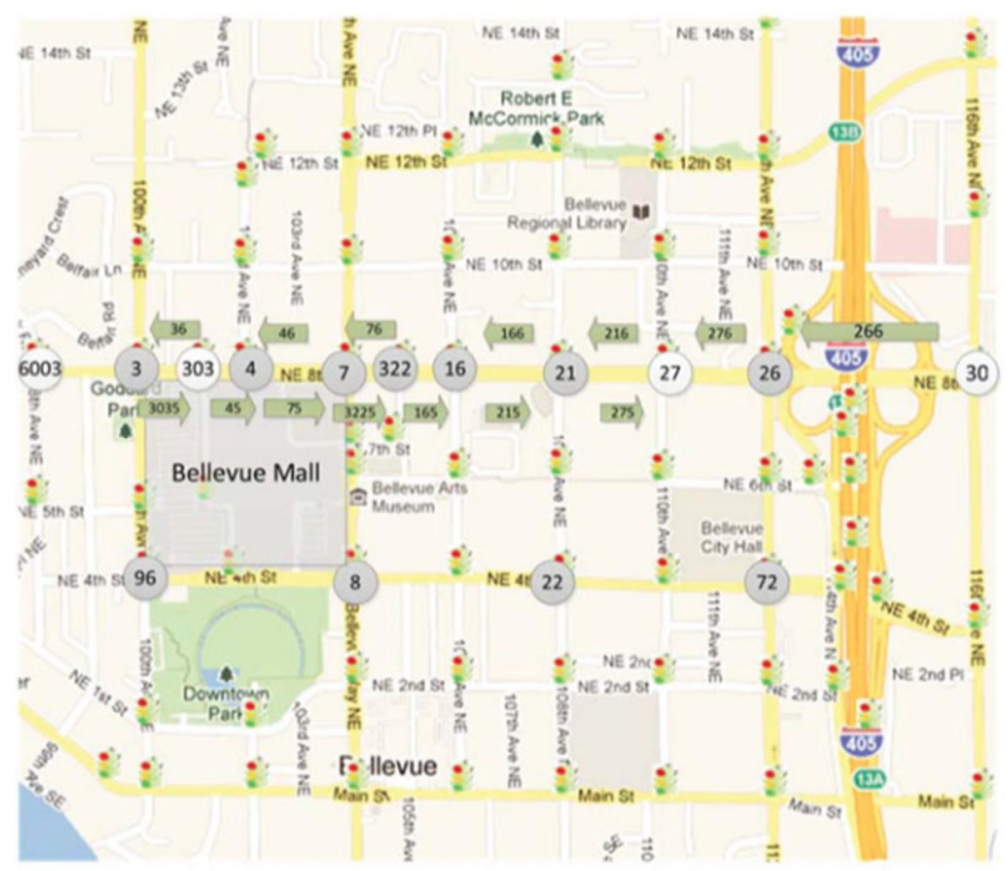

Read Article:

http://www.tandfonline.com/doi/full/10.1080/15472450.2015.1072050

\section{Dynamic Bandwidth Analysis for Coordinated Arterial Streets}

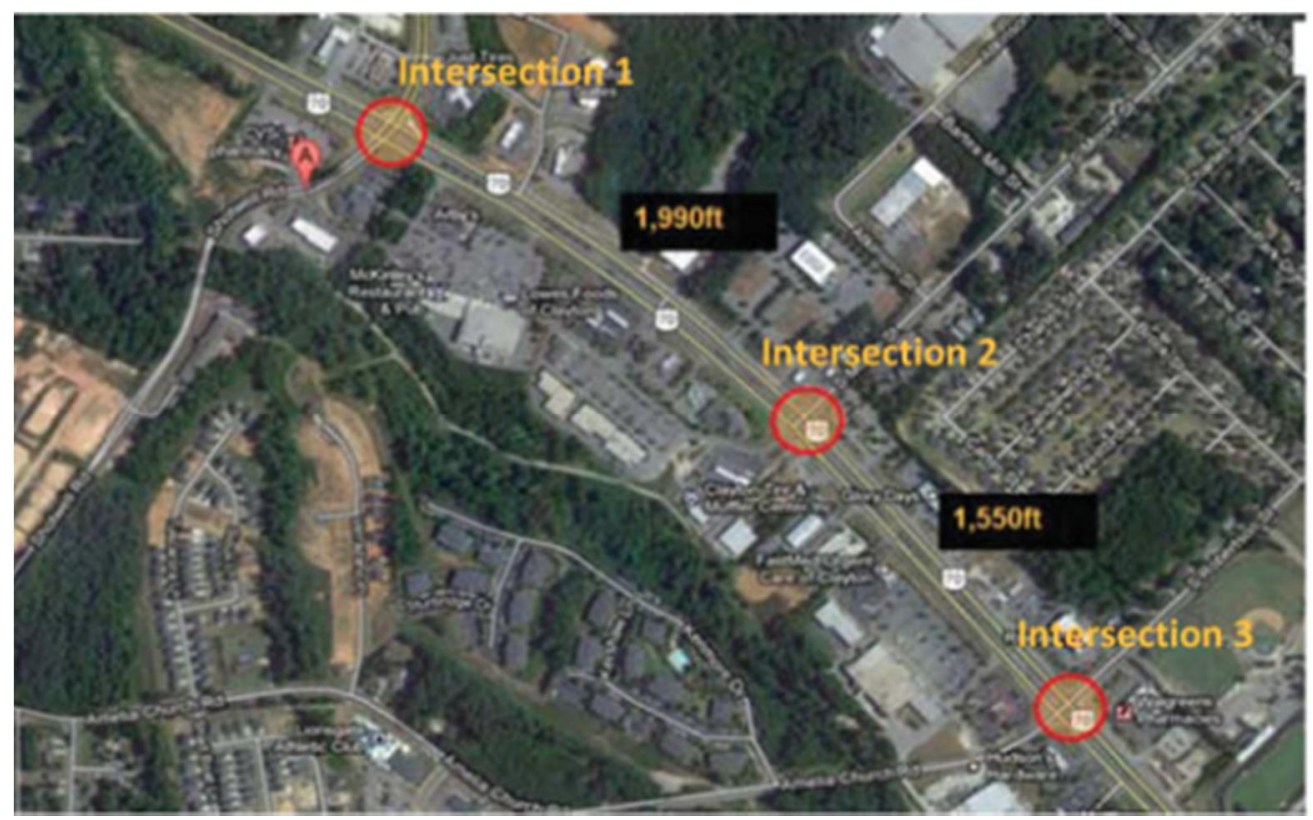

Read Article:

http://www.tandfonline.com/doi/pdf/10.1080/15472450.2015.1074575 
Incident management in intelligent transportation systems

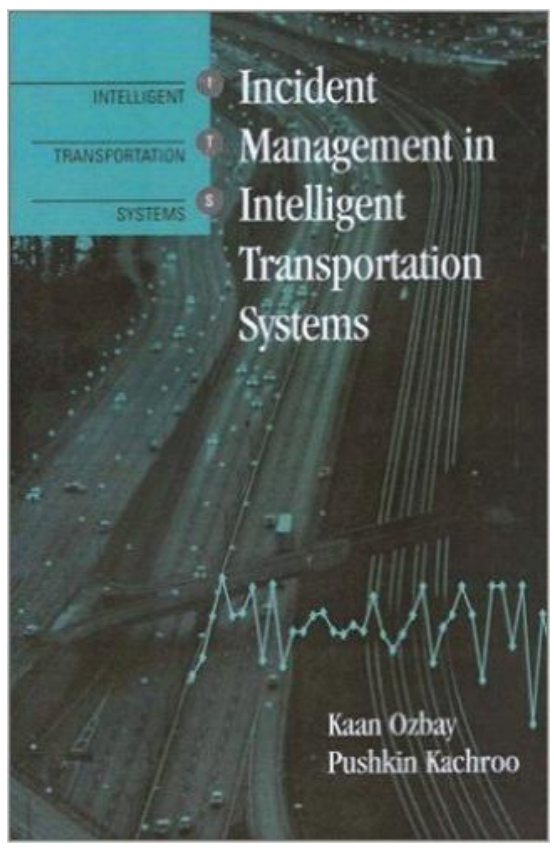

Read Article:

http://digitalscholarship.unlv.edu/ece_fac_articles/103/

Intelligent Transportation Systems: New Principles and Architectures

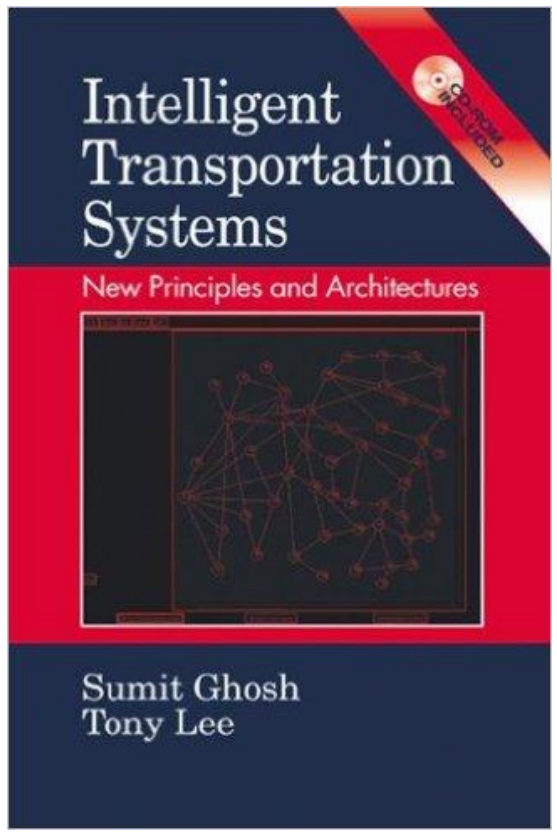

Read Article:

http://www.amazon.com/Intelligent-Transportation-Systems-Architectures-

Engineering/dp/0849300673 
Intelligent Transportation Systems Architectures

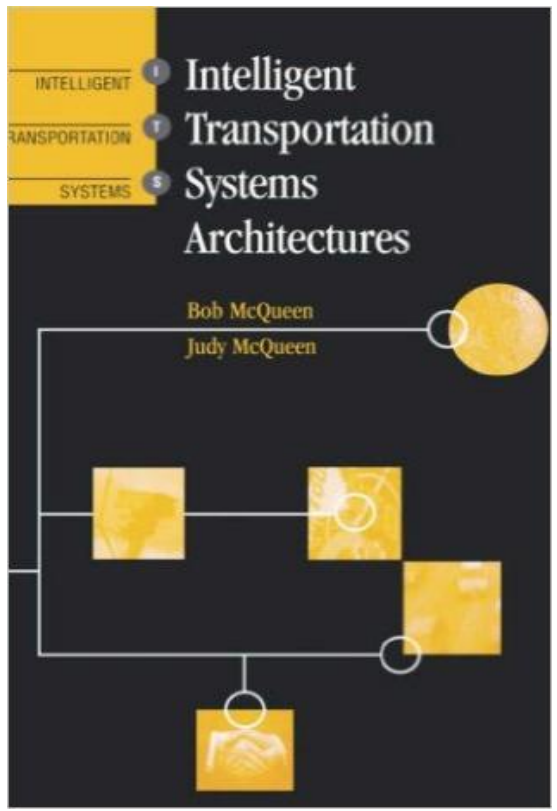

Read Article:

https://trid.trb.org/view.aspx?id=496910

Human Factors in Intelligent Transportation Systems

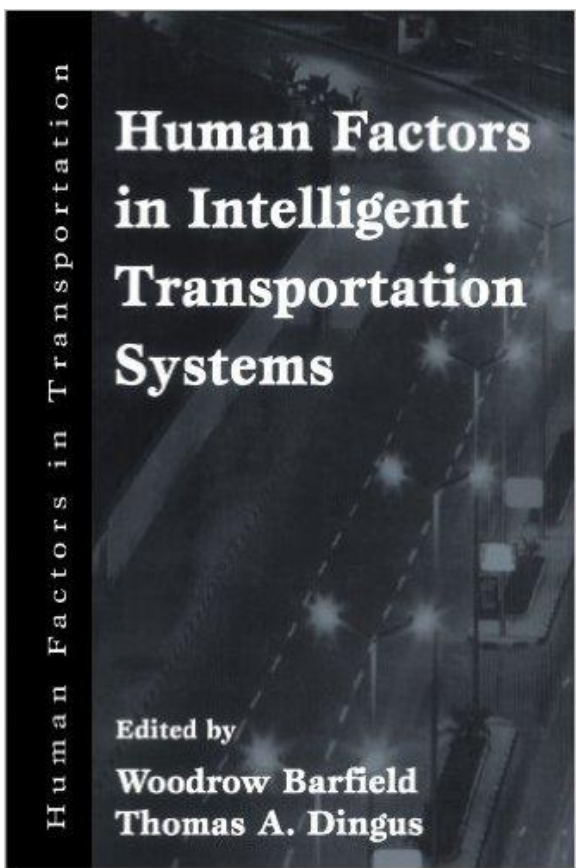

Read Article:

http://www.amazon.com/Human-Factors-Intelligent-Transportation-

Systems/dp/0805814345 
Positioning Systems in Intelligent Transportation Systems

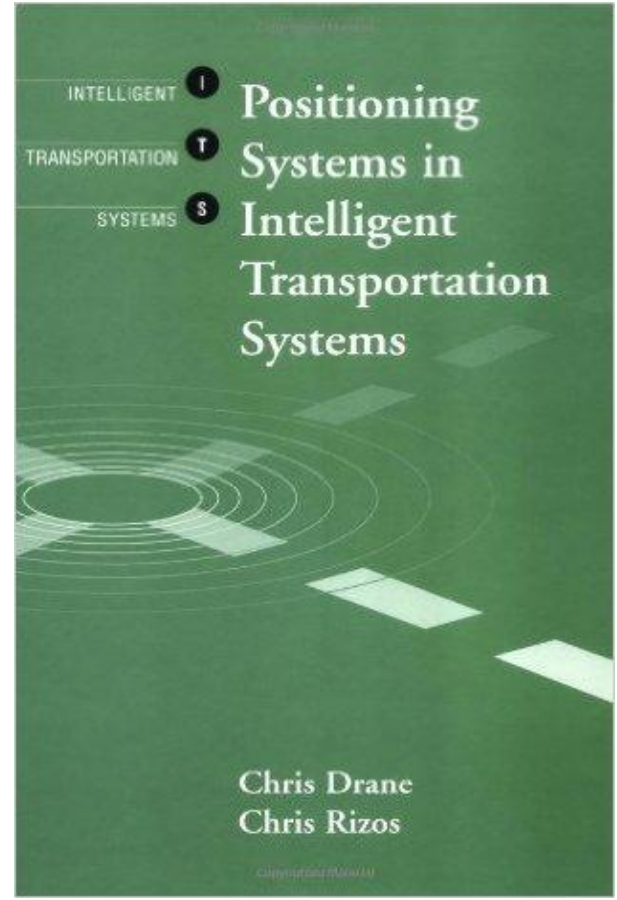

Read Article:

http://dl.acm.org/citation.cfm?id=550829

\section{Integrated Platform for Road Traffic Safety Data Collection and Information Management}

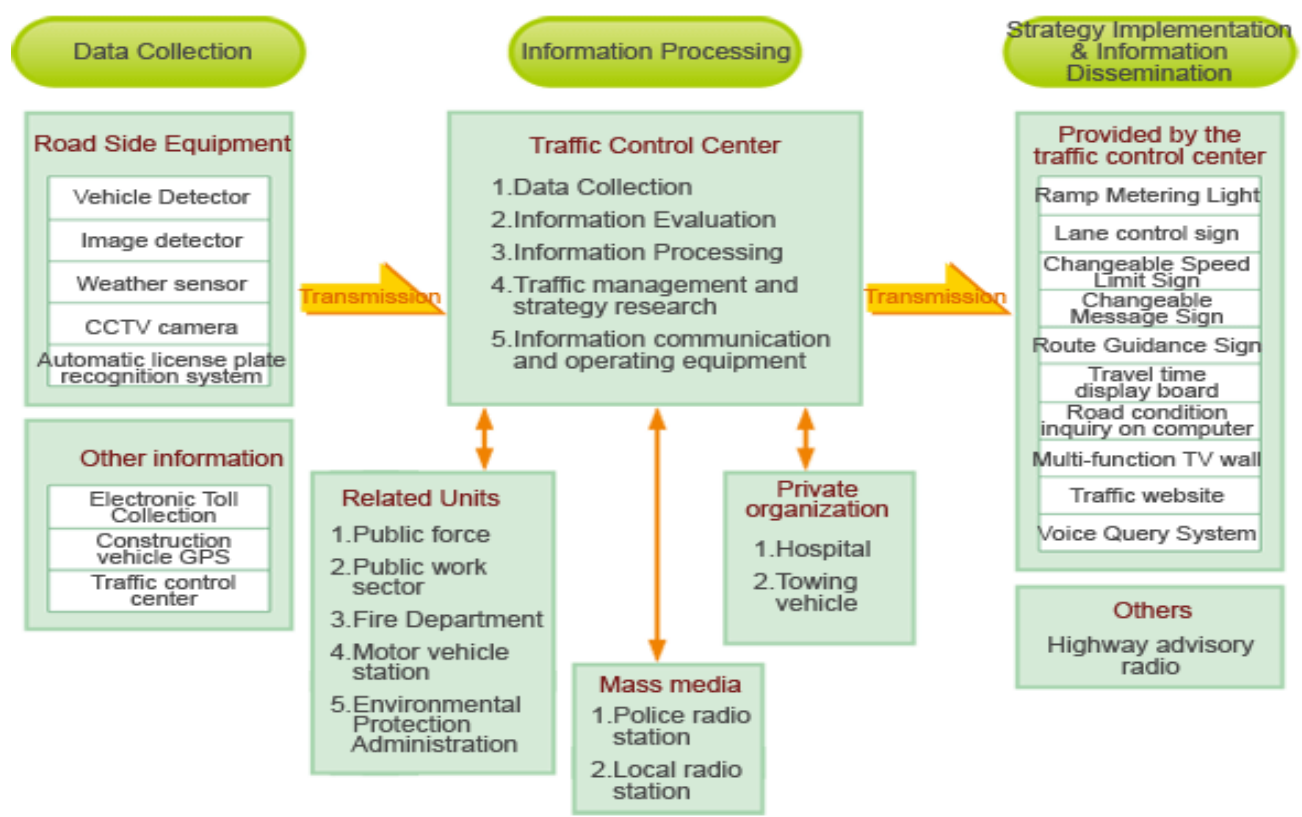

Read Article:

http://ieeexplore.ieee.org/xpls/icp.jsp?arnumber=5628904 
The smart way to manage traffic congestion

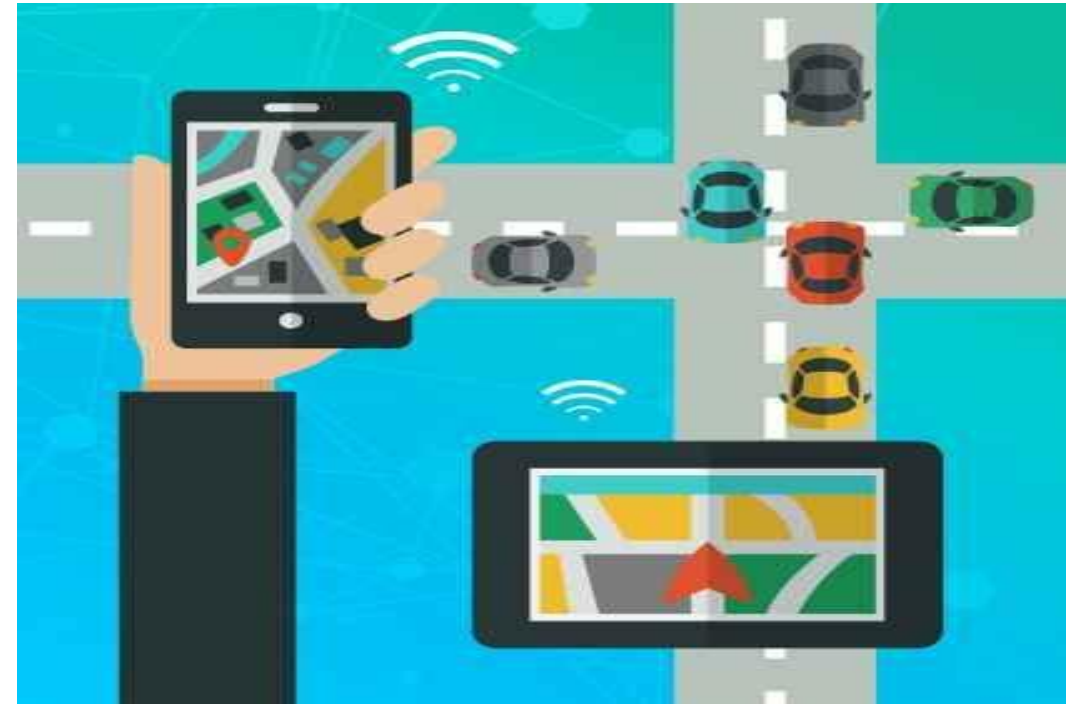

Read Article:

http://www.moneycontrol.com/digitizingindia/news/the-smart-way-to-manage-trafficcongestion-2580021.html

How digital road management could save the UK £300bn by 2030

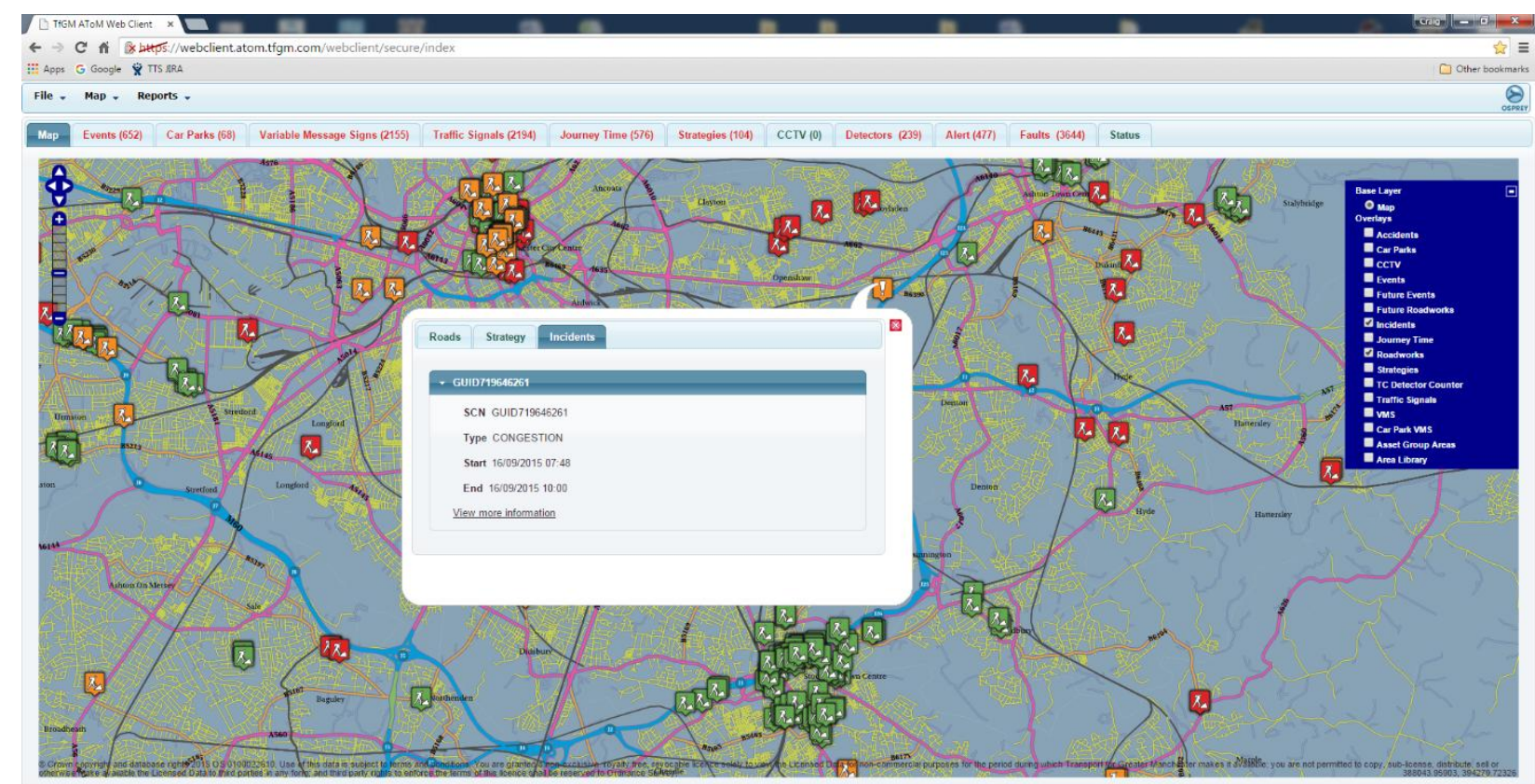

\section{Read Article:}

http://www.infrastructure-intelligence.com/article/nov-2015/how-digital-roadmanagement-could-save-uk-\%C2\%A3300bn-2030 
Departure Side Platforms: a road congestion mitigation measure

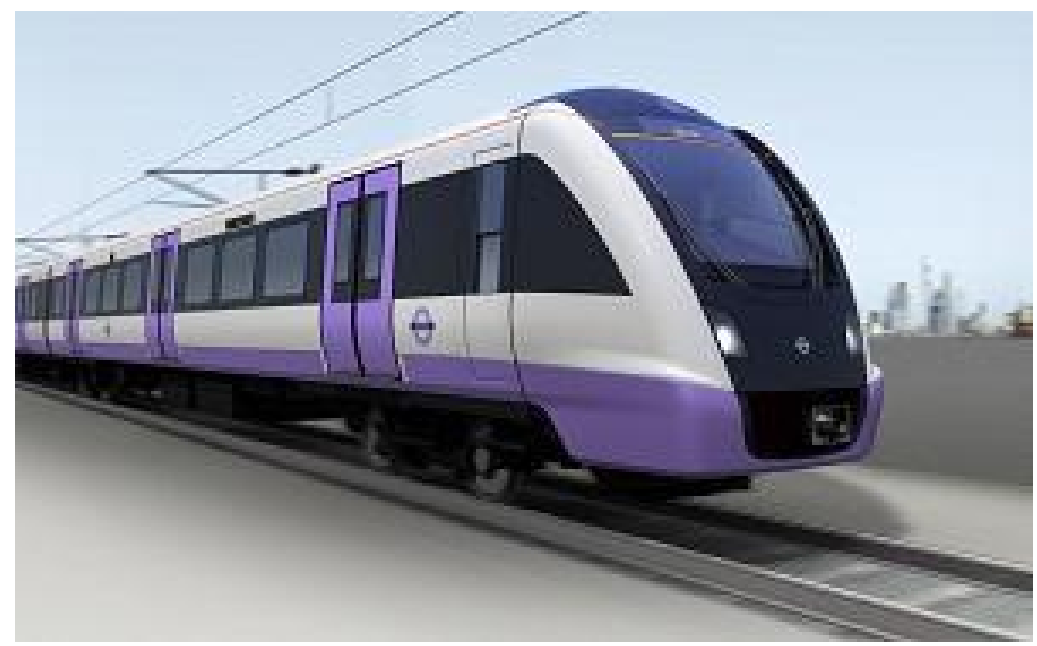

Read Article:

http://www.sidrasolutions.com/Cms_Data/Contents/SIDRA/Media/Articles/Guzman_CAI TR\%202015\%20Paper\%20.pdf

\section{Advanced Data Analytics in Transport - Machine Learning Perspective}

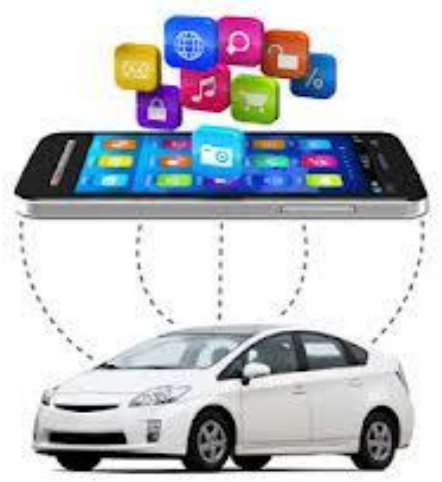

Read Article:

https://www.nicta.com.au/category/research/machine-learning/projects/advanced-dataanalytics-in-transport-machine-learning-perspective/ 
MEMS sensor solutions for loT in transport and buildings

\section{MEMS-based System Solutions (MBSS)}

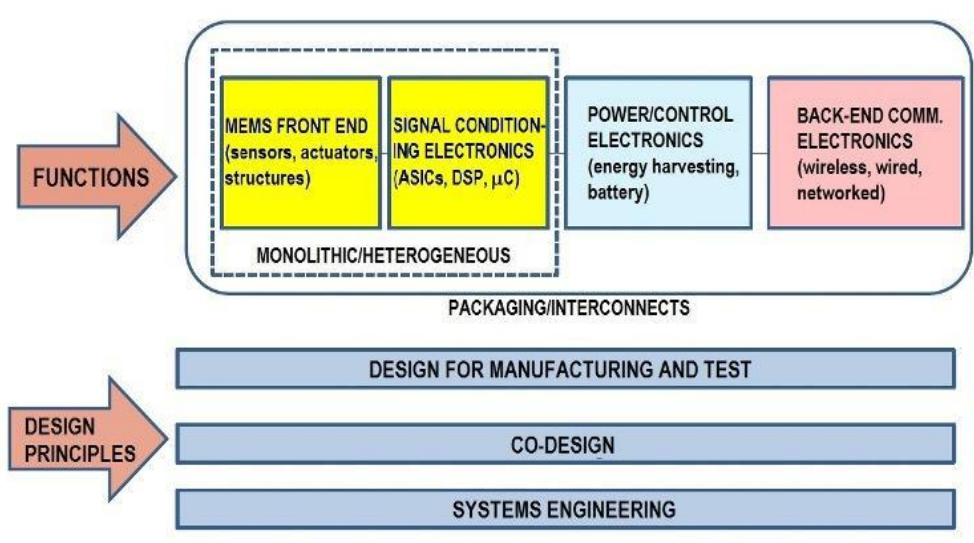

Read Article:

http://www.electronicproducts.com/Test_and_Measurement/Benchtop_Rack_Mountable/ MEMS_sensor_solutions_for_loT_in_transport_and_buildings.aspx

Digital platform more than taxi service: Uber as app economy paradigm?

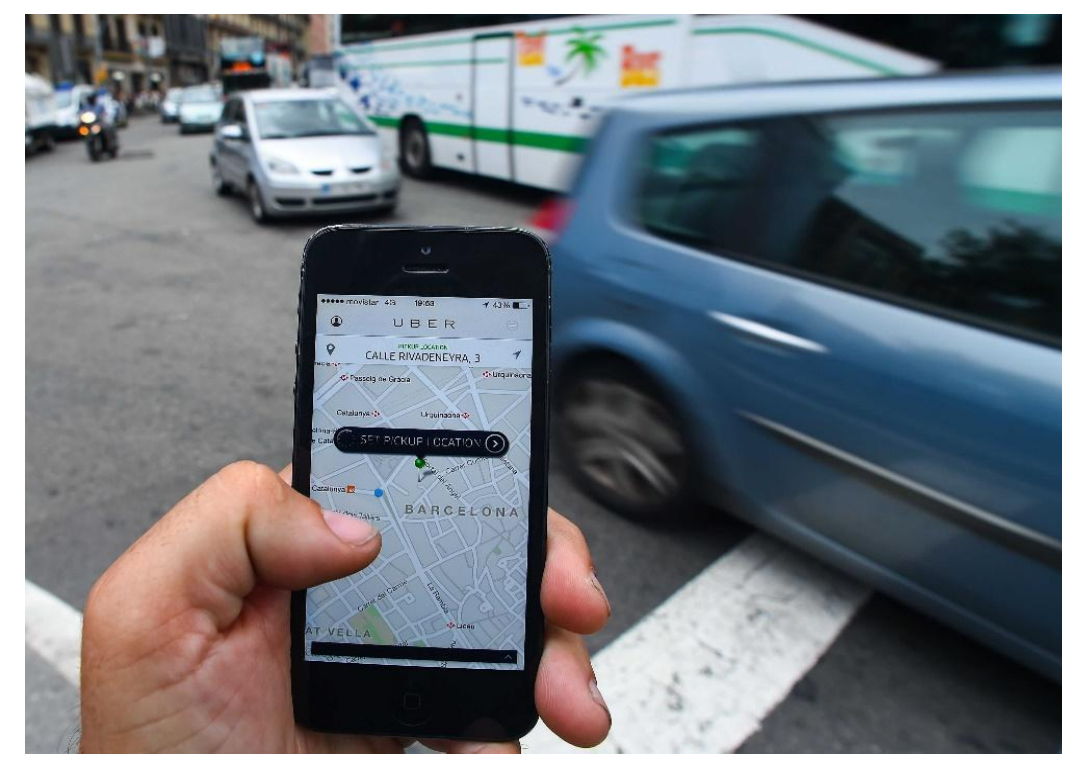

Read Article:

http://www.computerweekly.com/opinion/Digital-platform-more-than-taxi-service-Uberas-app-economy-paradigm 
First Steps toward Drone Traffic Management

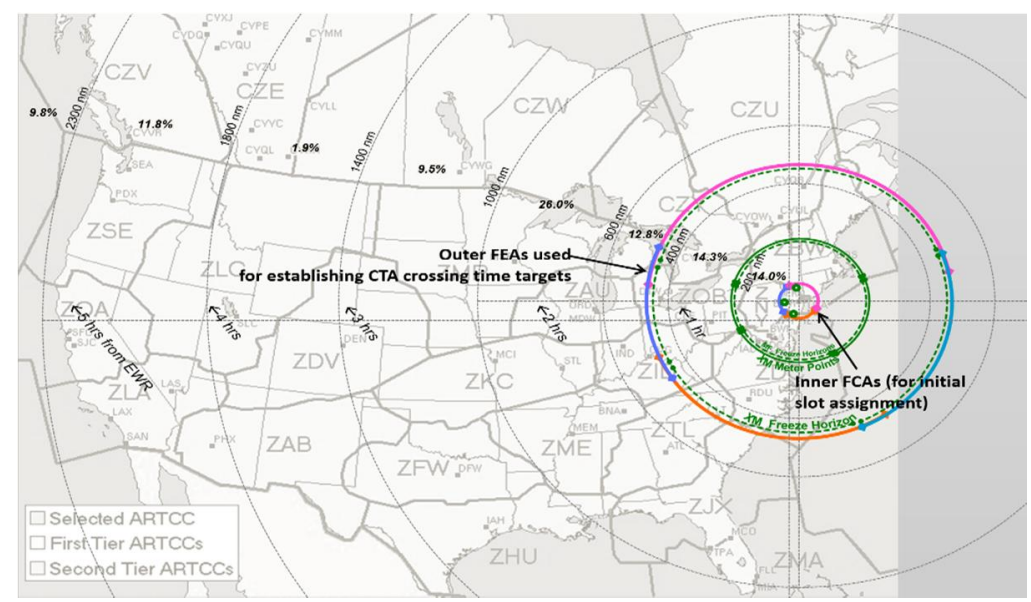

Read Article:

http://www.nasa.gov/feature/ames/first-steps-toward-drone-traffic-management

\section{A framework for artificial transportation systems: From computer simulations to computational experiments}

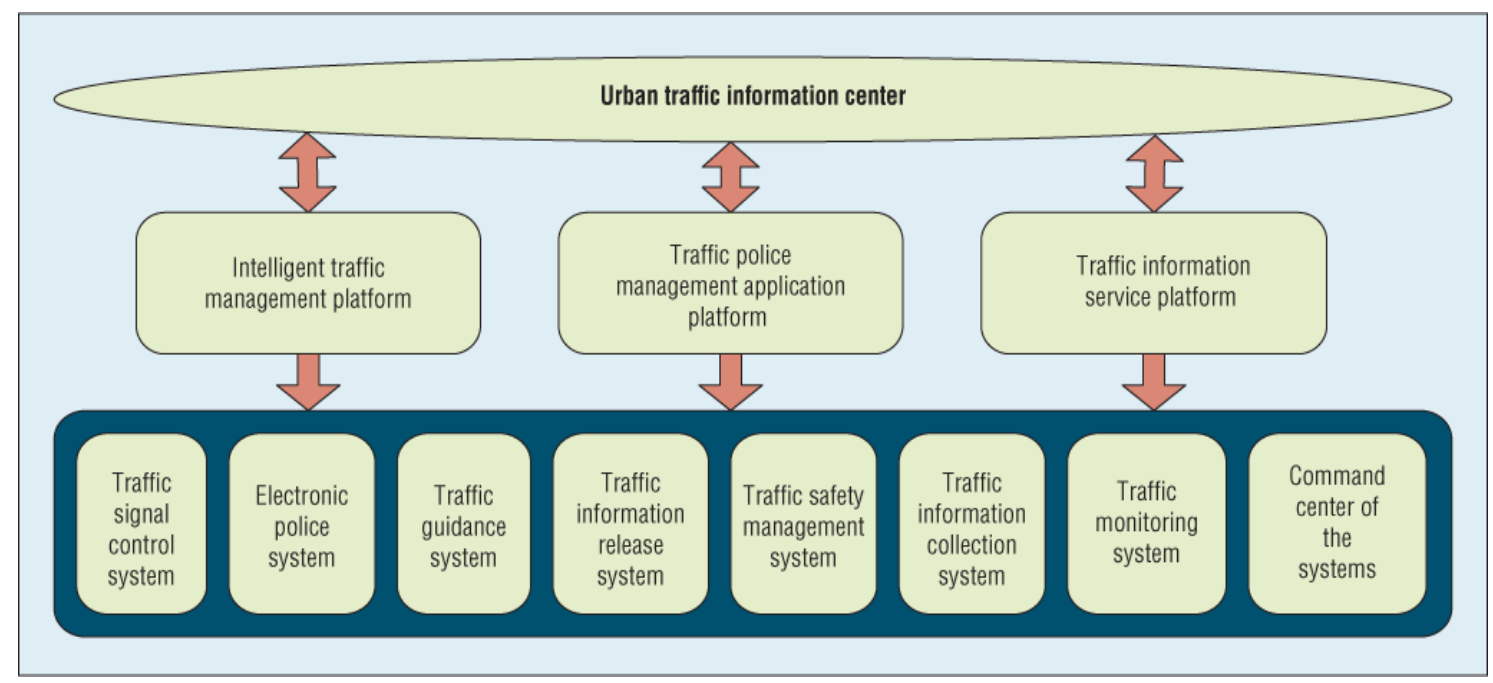

Read Article:

http://ieeexplore.ieee.org/xpls/icp.jsp?arnumber=1520210 
Real Time Simulation Platform Design of Traffic Control Hardware In-The-Loop

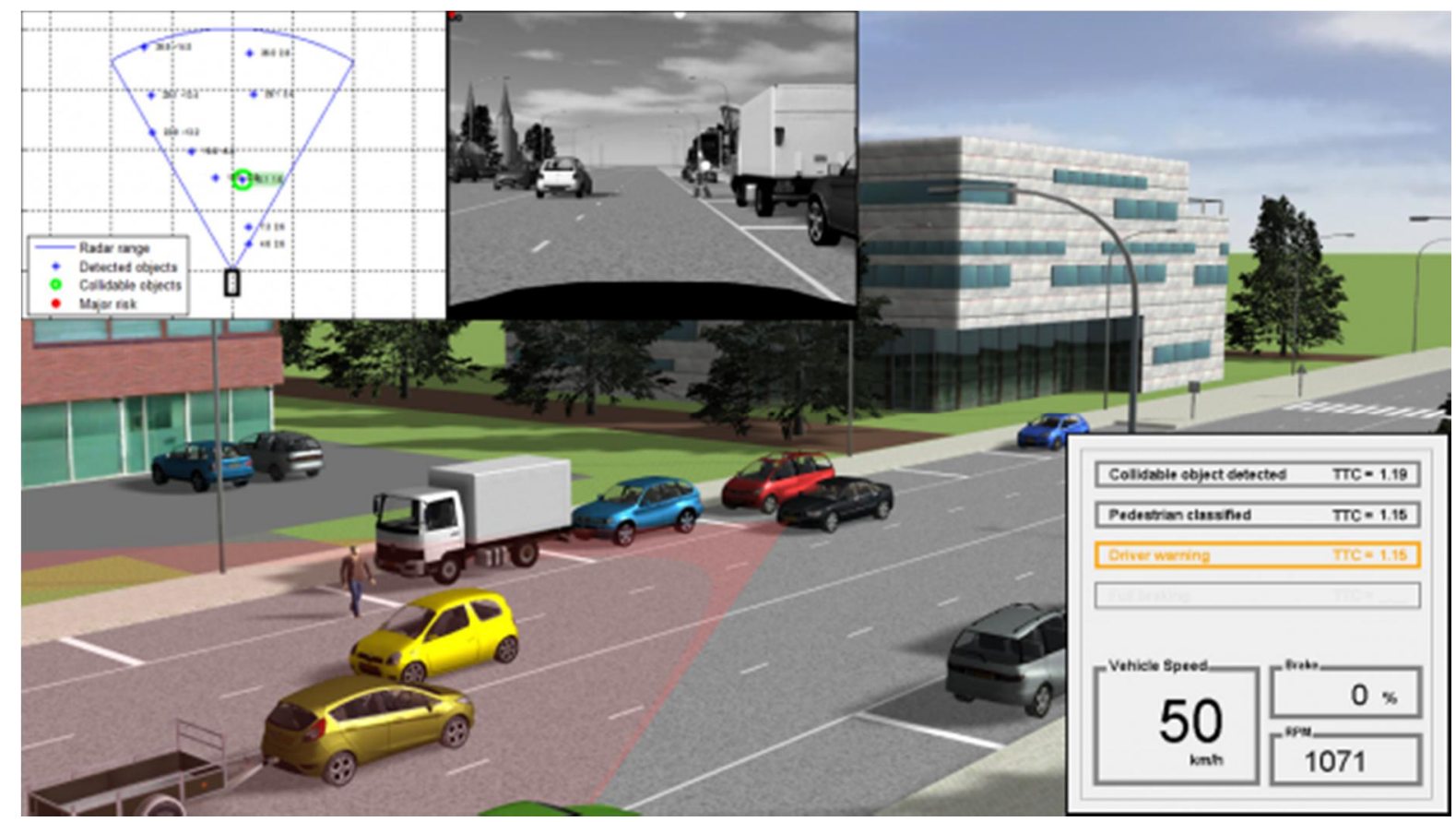

Read Article:

http://en.cnki.com.cn/Article_en/CJFDTOTAL-CGGL200910013.htm

Emerging transport technologies: Assessing impacts and implications for the City of Melbourne

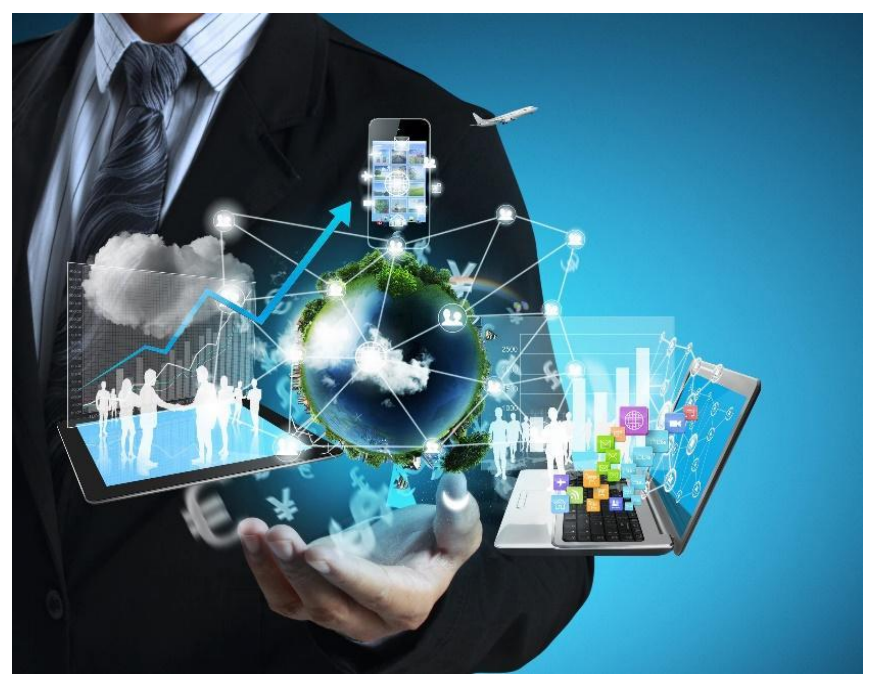

Read Article:

http://www.melbourne.vic.gov.au/SiteCollectionDocuments/emerging-transporttechnologies-report.pdf 
Predictable Disruption: Looking to digital ecosystems for the next waves of change

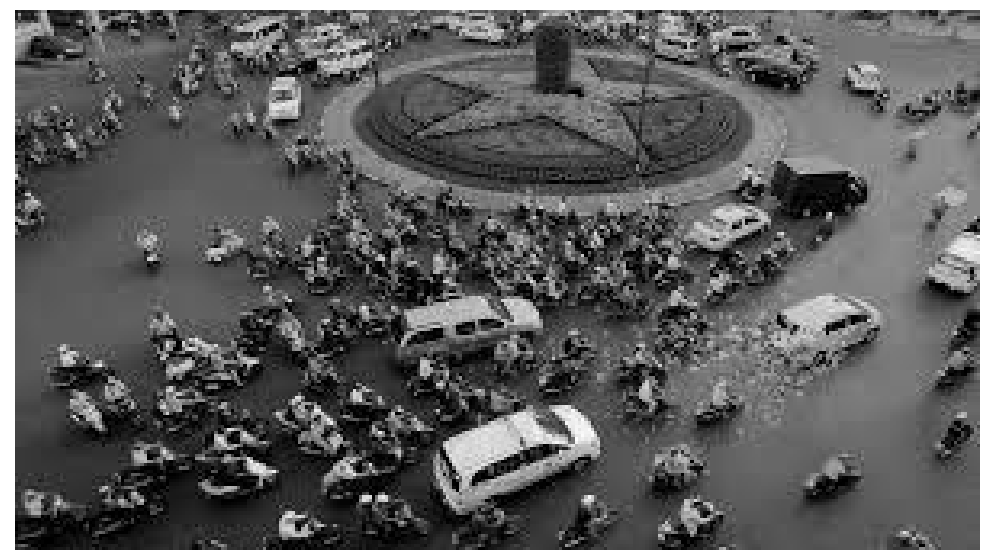

Read Article:

https://www.accenture.com/fr-fr/_acnmedia/PDF-2/Accenture-Predictable-DisruptionTechnology-Vision-2016-france.pdf

A Road Traffic Survey Platform Application of "Reference Road Segments and Reference Intersections"

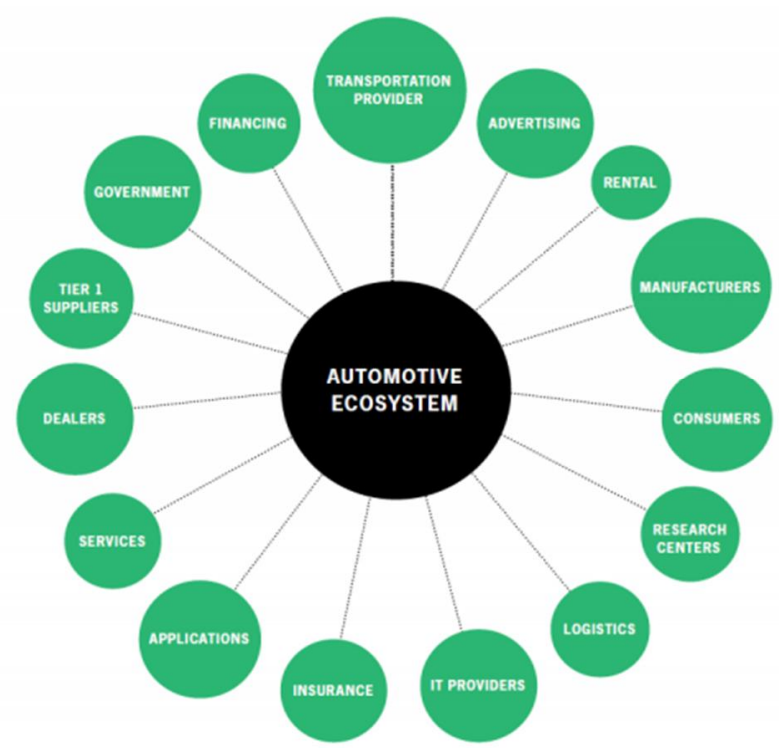

Read Article:

http://www.nilim.go.jp/english/annual/annual2012/83.pdf 
Relieving Traffic Congestion by Tracking Vehicle Movement: Leveraging Locus Data to Help Make Roads Safer and Better

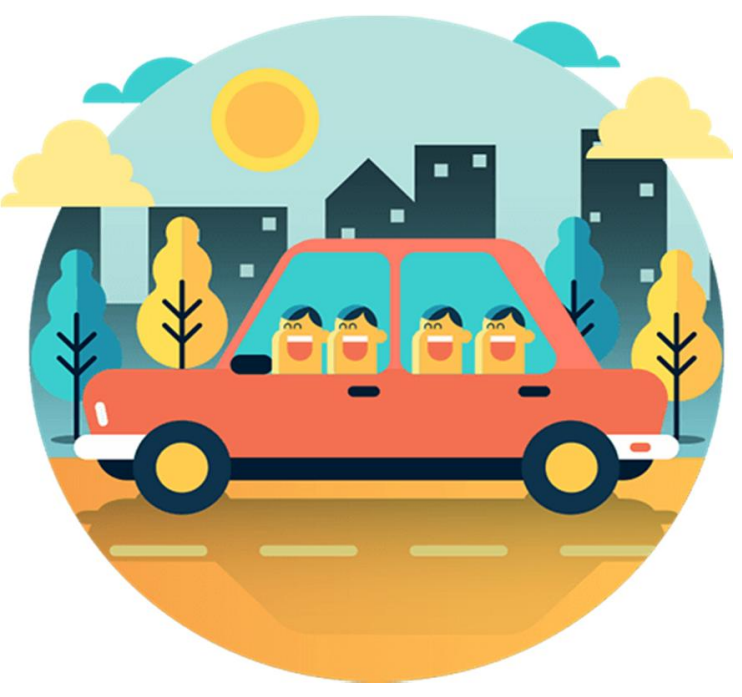

Read Article:

http://journal.jp.fujitsu.com/en/2015/08/27/01/

Transforming transport by building ecosystems, not egosystems

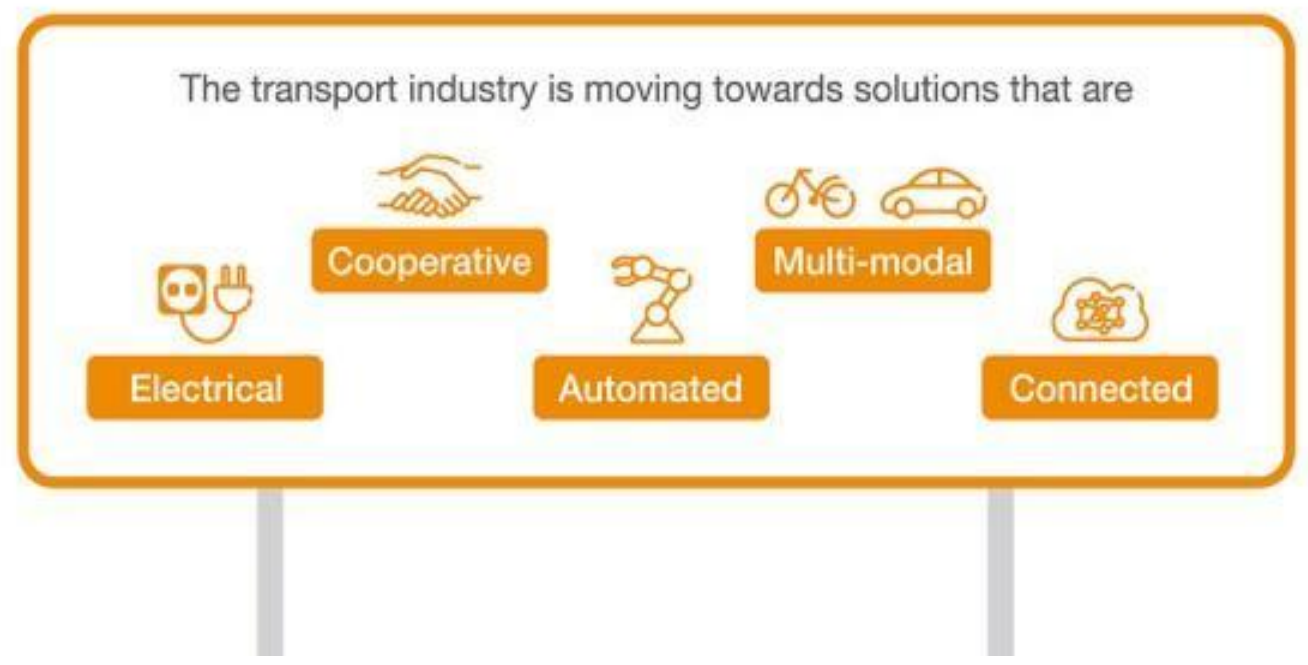

Read Article:

http://www.newcitiesfoundation.org/transforming-transport-by-building-ecosystems-notego-systems/ 


\section{Better transportation customer experiences through digital}

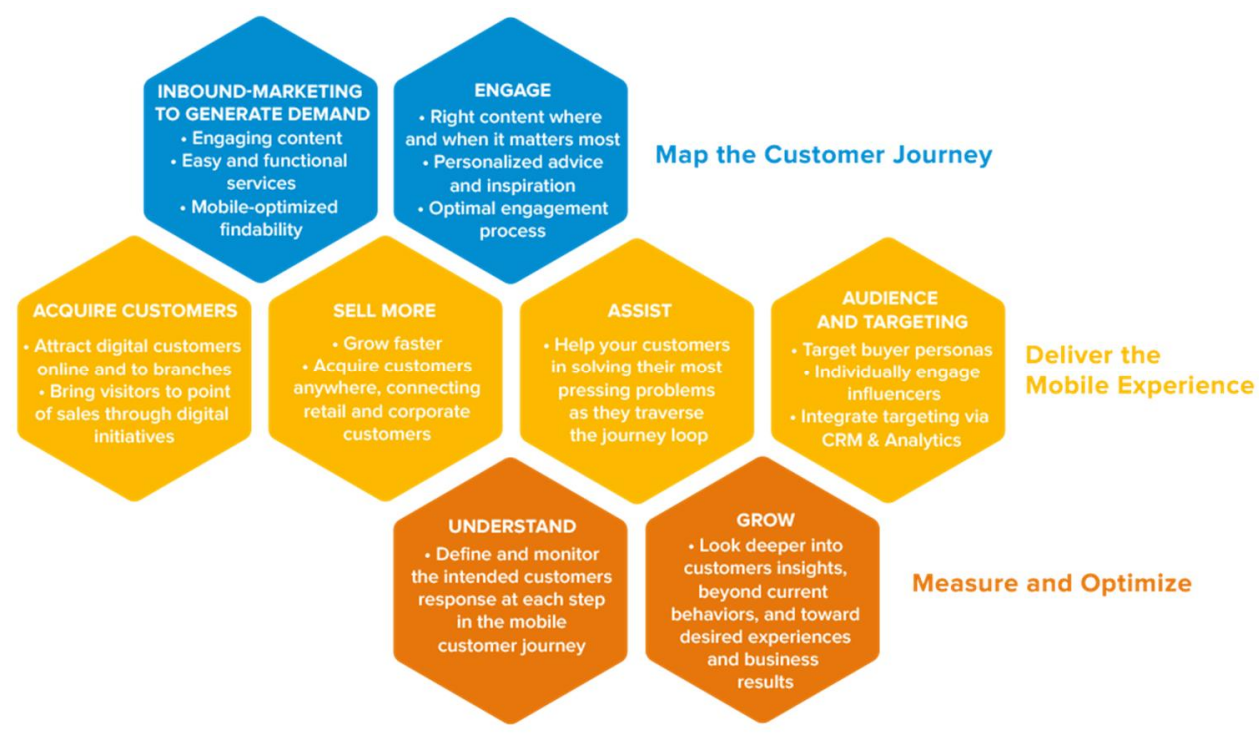

Read Article:

https://www.digitalpulse.pwc.com.au/transport-information-ecosystem-partners-platformapproach-and-targeted-benefits/

\section{Technology and digital high street}

\section{Transport Imperatives}

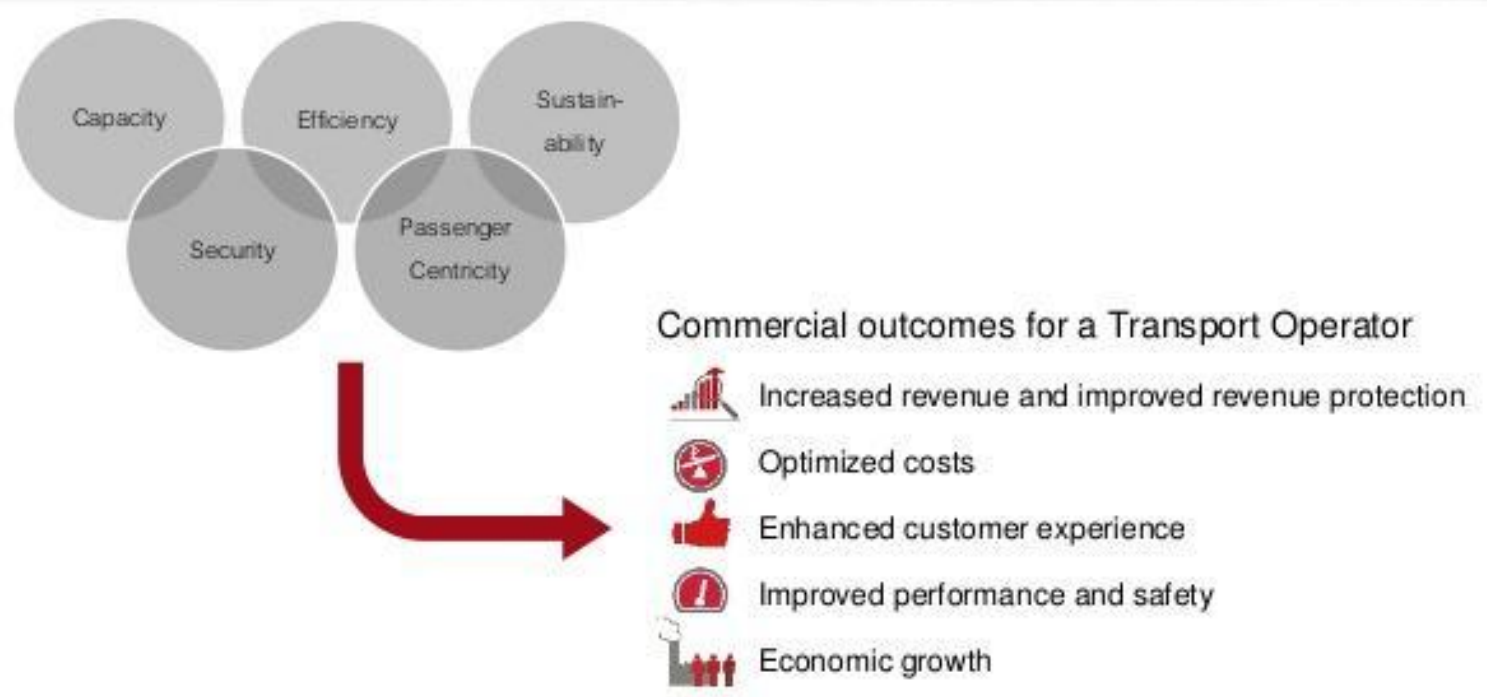

Read Article:

http://romanroadtrust.co.uk/technology-digital-high-street/ ' 
Self-driving cars are about platforms, not about cars

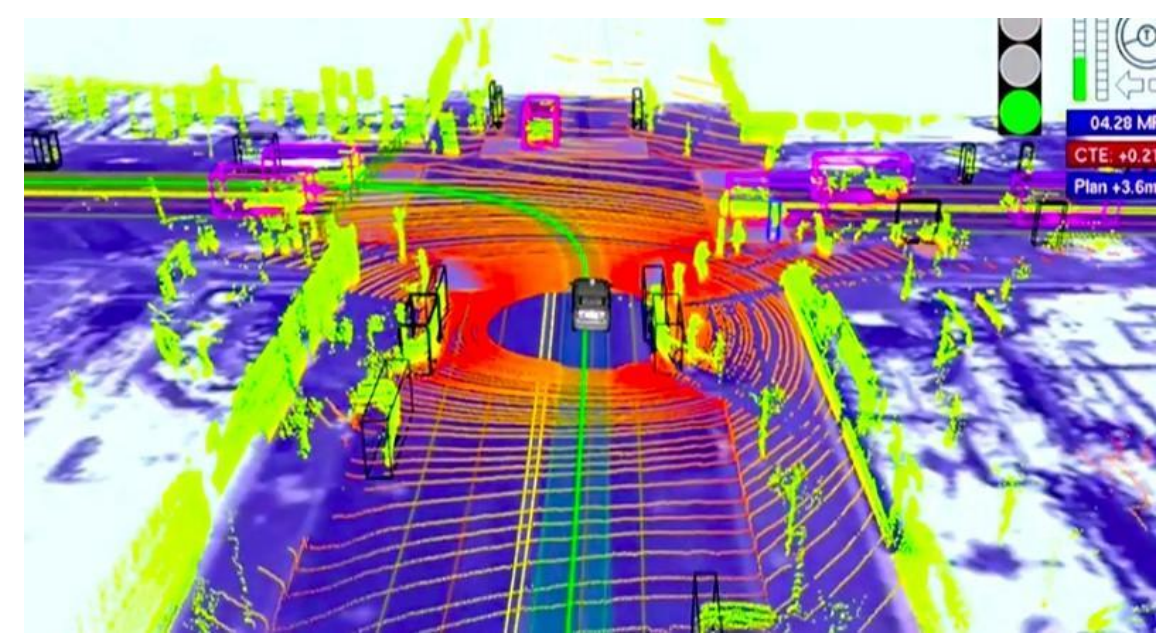

Read Article:

http://www.visionmobile.com/blog/2015/11/self-driving-cars-are-about-platforms-notabout-cars/

Research on the Platform Construction of Road Freight Market in China

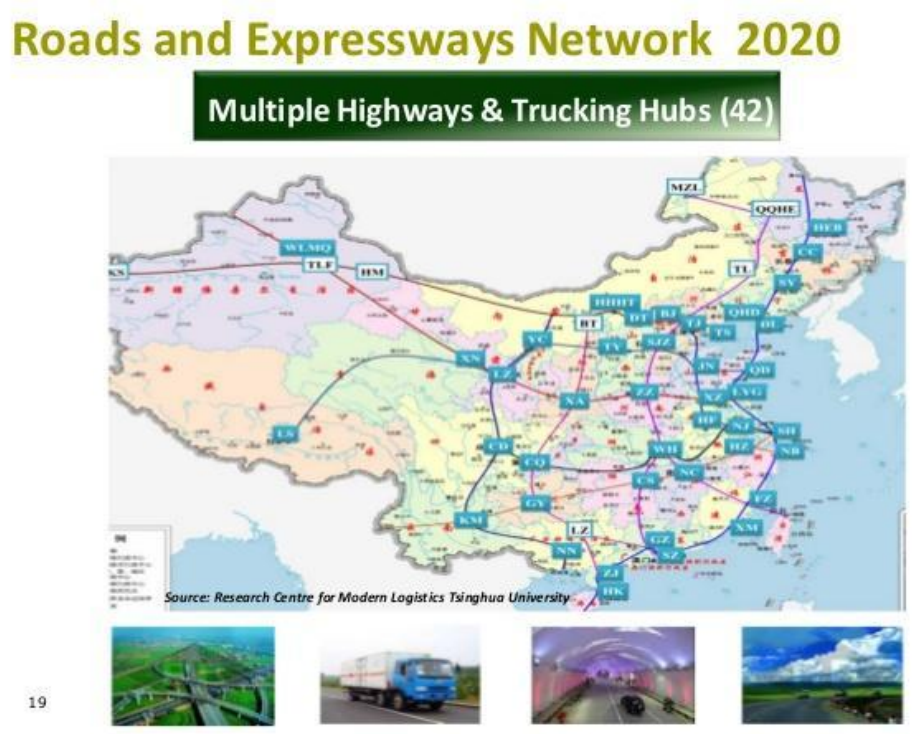

Read Article:

http://file.scirp.org/pdf/OJBM_2015061215475299.pdf 


\section{A WIDER SHARING ECOSYSTEM The pivotal role of data in transport solutions}

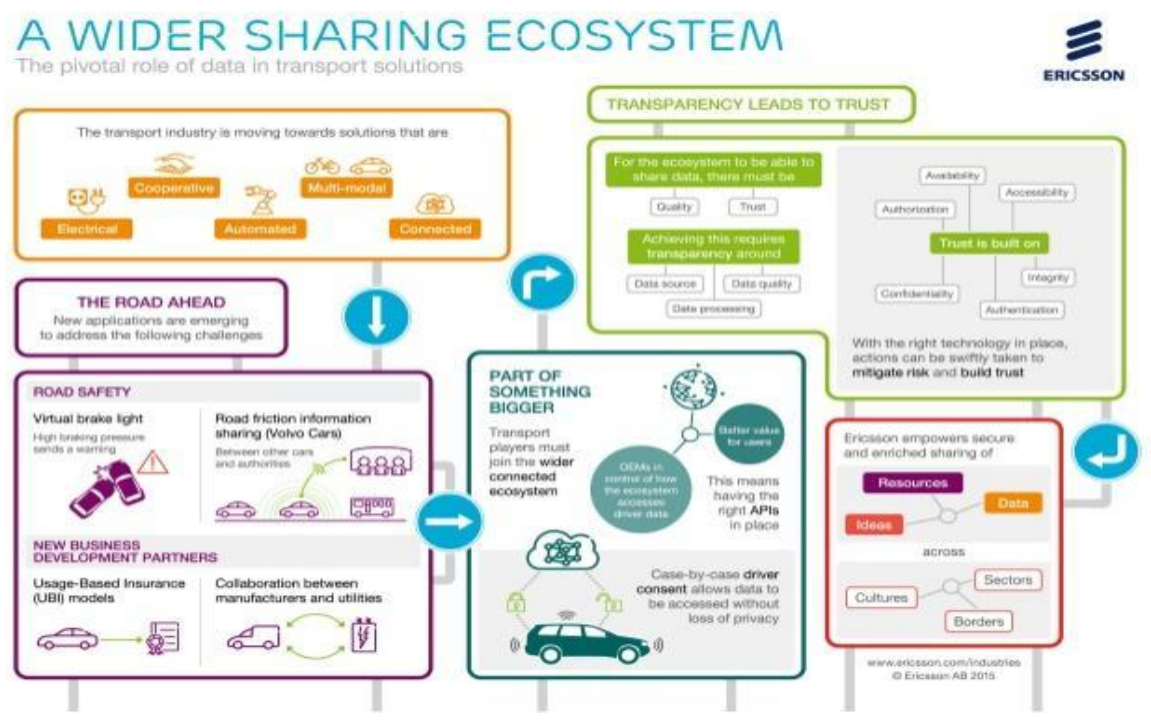

Read Article:

https://www.ericsson.com/res/docs/2015/a-wider-sharing-ecosystem.pdf

Data61 is building a transport analytics platform for the states

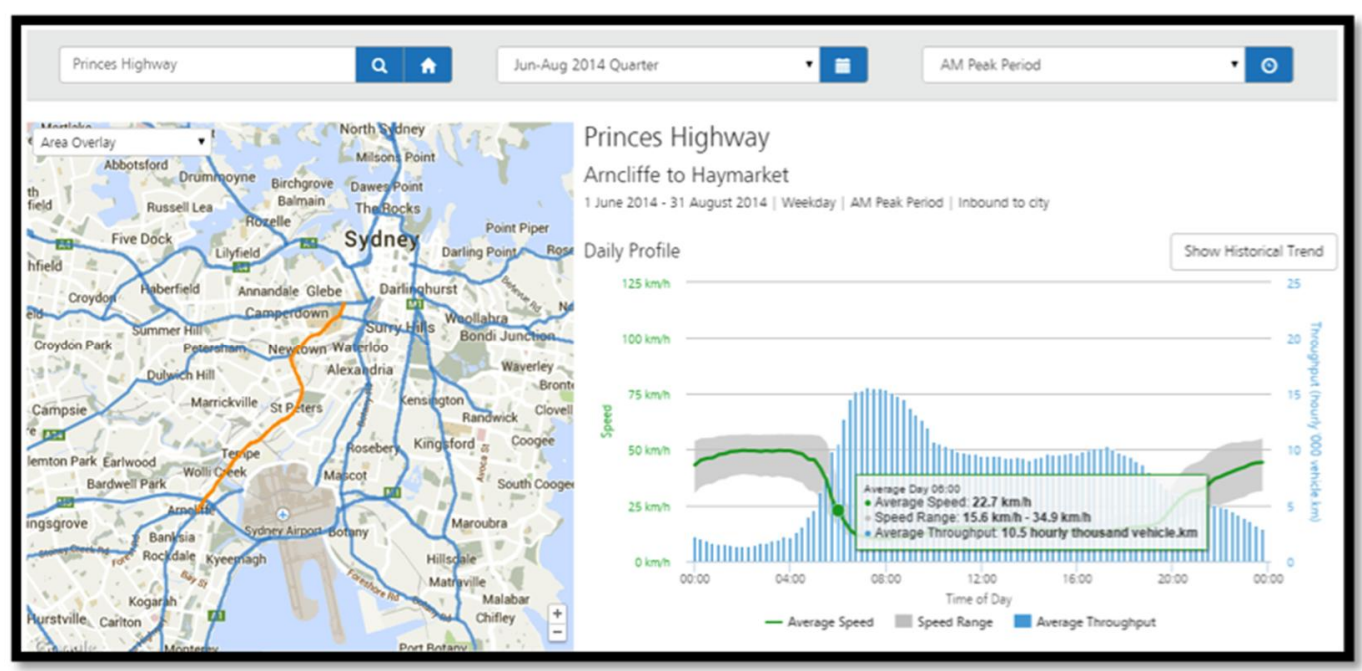

Read Article:

http://www.itnews.com.au/news/data61-is-building-a-transport-analytics-platform-for-thestates-418539 
TIMON ("Enhanced real time services for an optimized multimodal mobility relying on cooperative networks and open data")

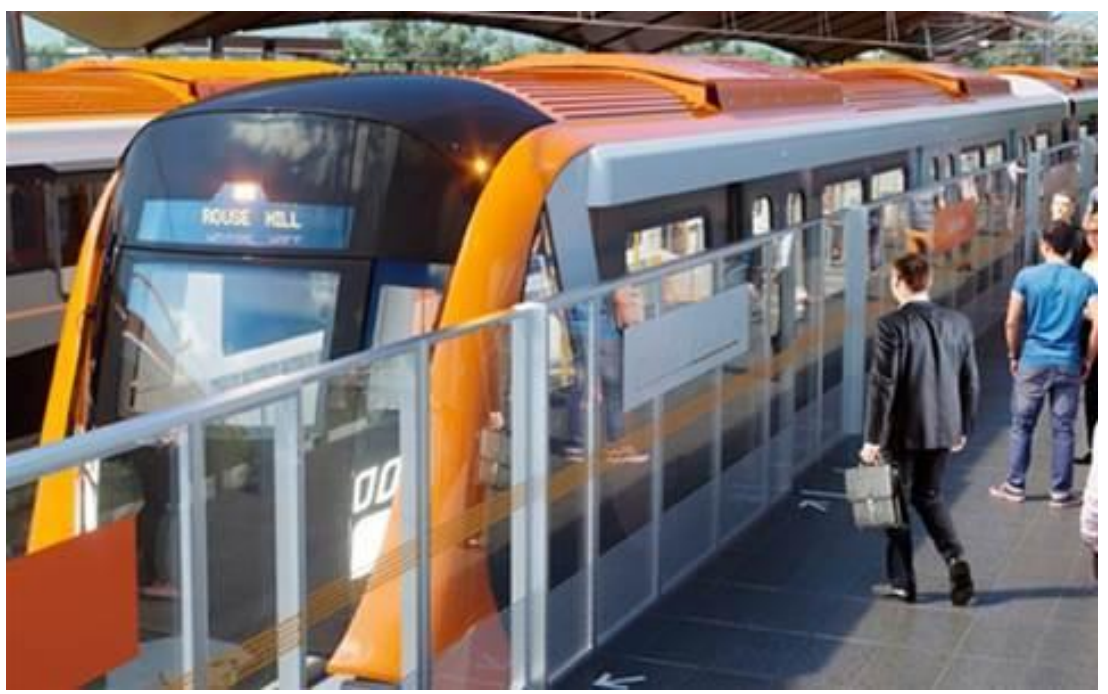

Read Article:

http://www.timon-project.eu/index.php/about-timom/what-is-timon.html

Innovative Transport Services in Finland

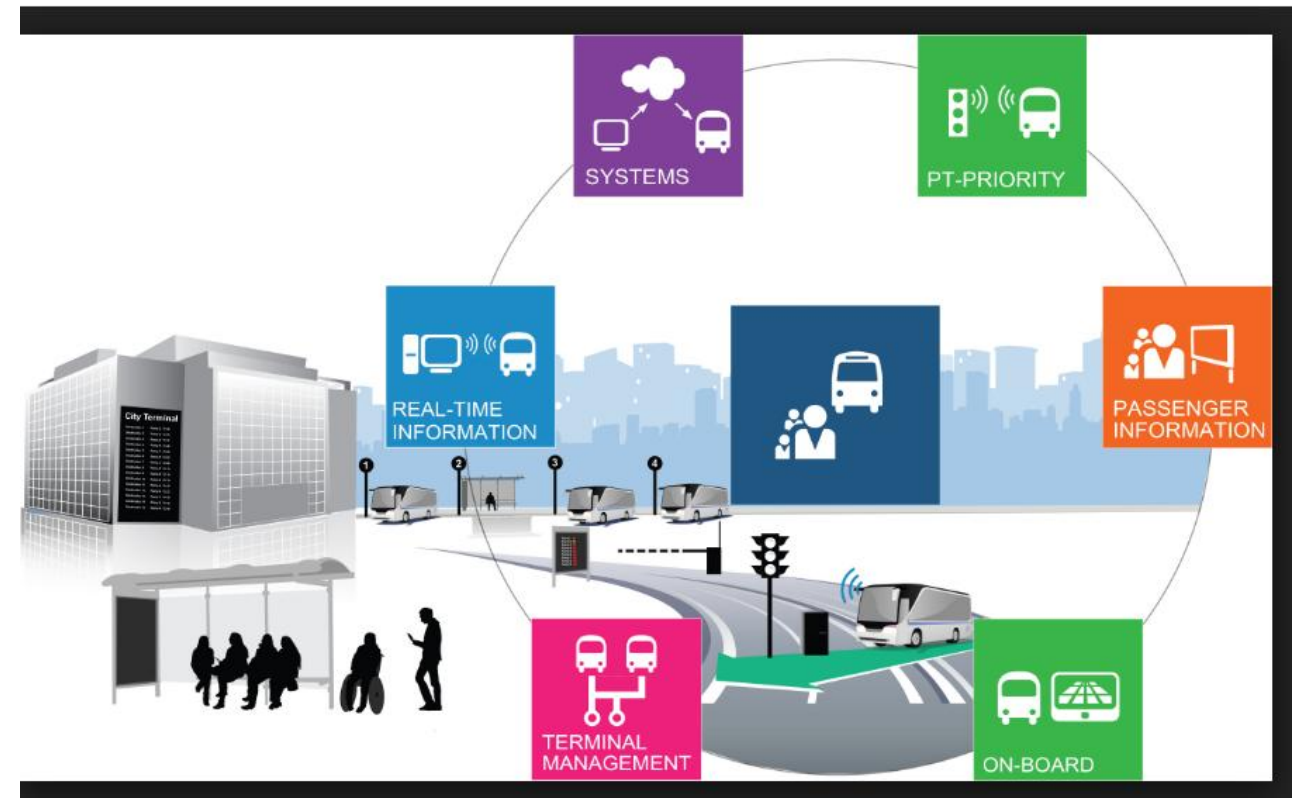

Read Article:

https://www.b2match.eu/system/eurostarsmatch/files/Raine_Hermans_Tekes_Hermans _MaaS_ecosystem.pdf?1460349835 
Navigating a Shifting Landscape: Capturing Value in the Evolving Mobility Ecosystem

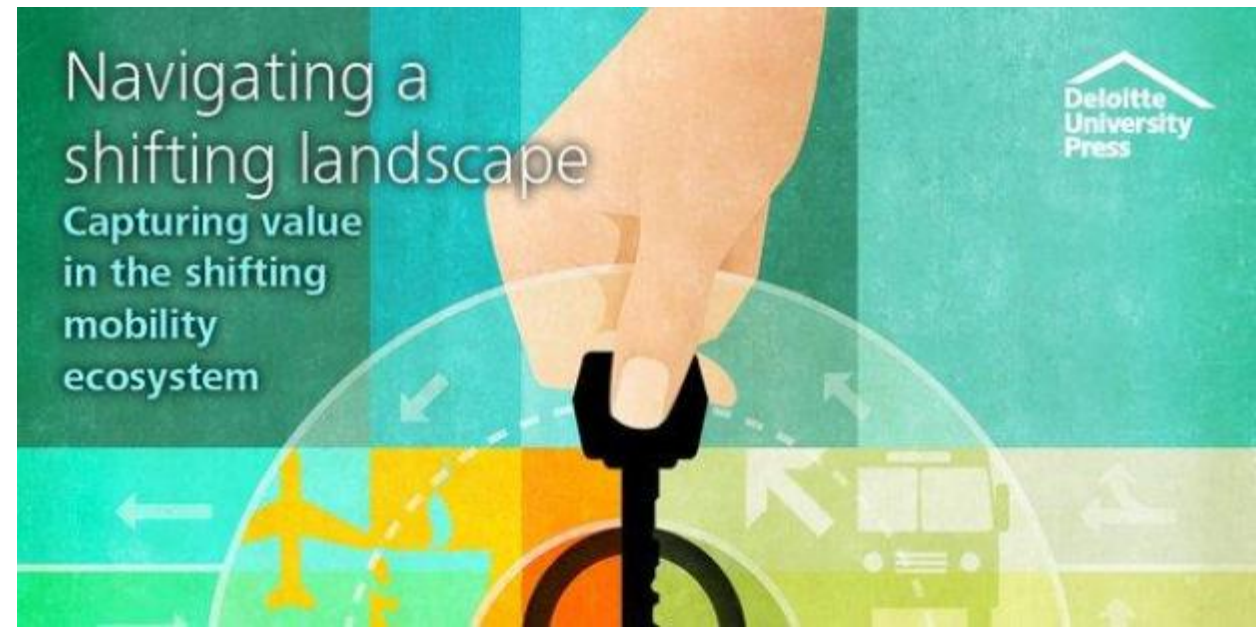

Read Article:

http://dupress.com/articles/future-transportation-technology-mobility-ecosystems/

\section{THE ROLE OF TRANSPORT TECHNOLOGY PLATFORMS IN FOSTERING EXPLOITATION}

\section{BIG SHIFTS TO ENABLE INNOVATION}

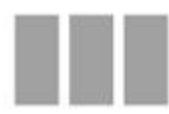

On-premises data centres

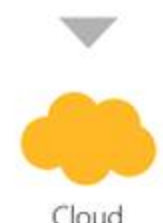

Cloud
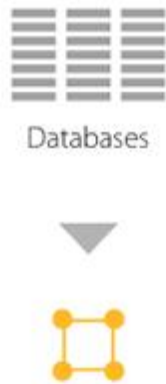

Data models

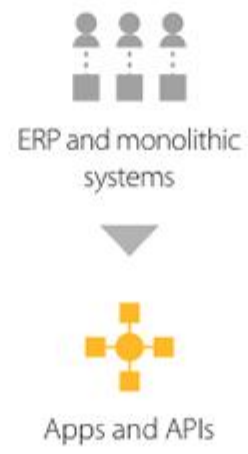

Apps and APIs

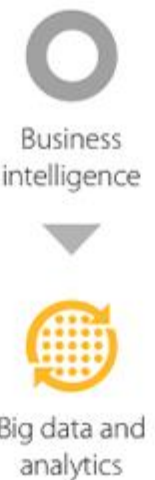

analytics

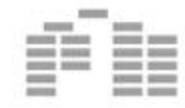

IT department

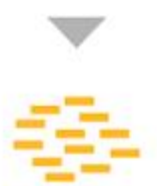

Networked IT structure

Read Article:

http://www.transport-tips.eu/uploads/files/TIPS_2nd_Training_Academy_4-

6Feb14_Lyon/14_TIPS_role_eu-technology-platforms_MIKULIK.pdf 
The Emergence of the German Digital Autobahn Ecosystem

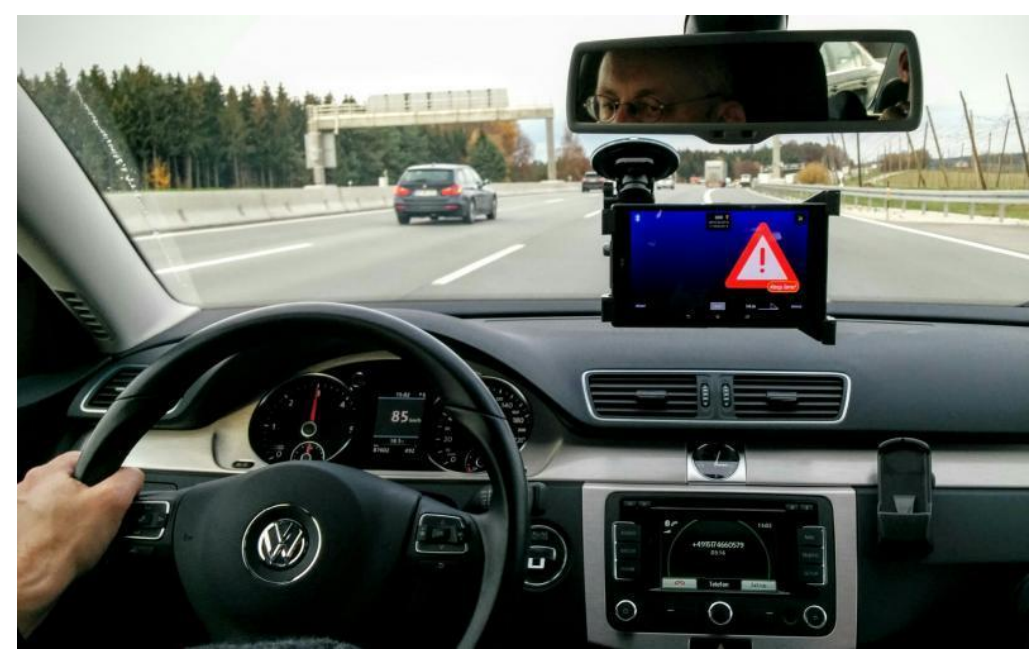

Read Article:

http://blogs.forrester.com/dan_bieler/15-11-11-

the_emergence_of_the_german_digital_autobahn_ecosystem

The Coming Fourth Digital Revolution in the Travel Ecosystem

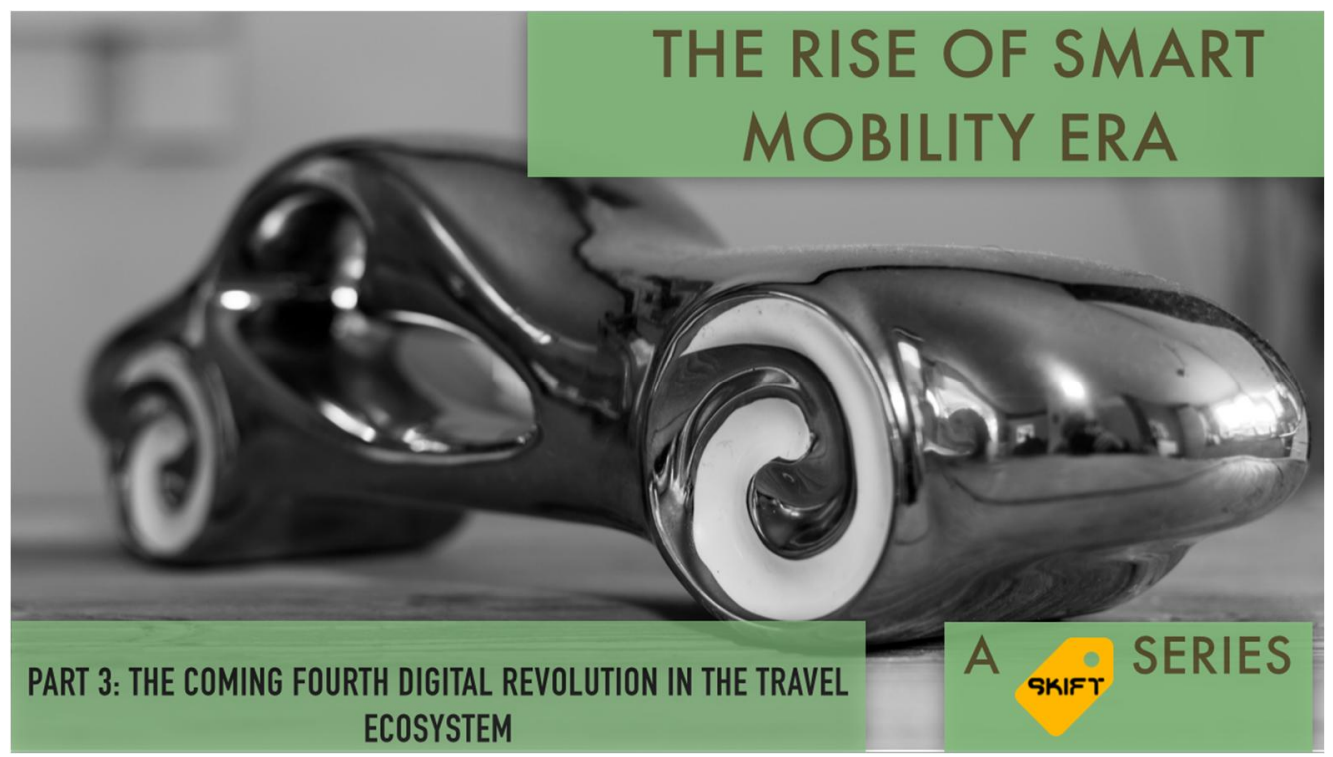

Read Article:

https://skift.com/2015/05/04/the-coming-fourth-digital-revolution-in-the-travel-ecosystem/ 
Transport for London creates an open data ecosystem with Amazon Web Services

\section{The power of open data on AWS}

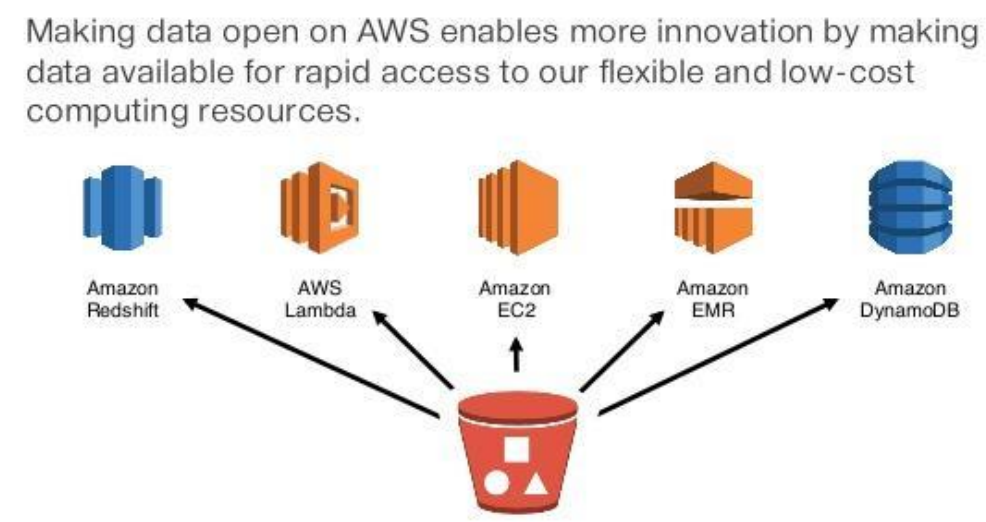

Read Article:

https://d0.awsstatic.com/analyst-reports/MWD_AWS_TFL_Case_Study_Sept_2015.pdf

\section{Business Strategy: Harnessing Connected Vehicle Ecosystem B2X Opportunities}

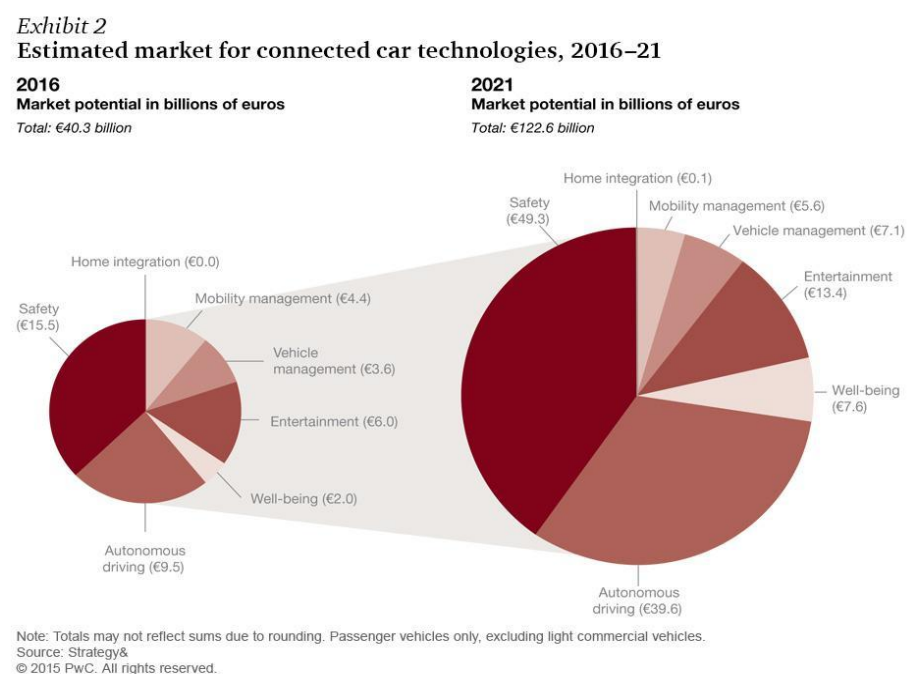

Read Article:

http://www.idc.com/prodserv/insights/manufacturing/downloads/IDC-MI-HarnessingConnected-Vehicles-Ecosystem-B2X-Opportunties.pdf 


\section{Apps for transport maps: connecting the dots in a complex ecosystem}
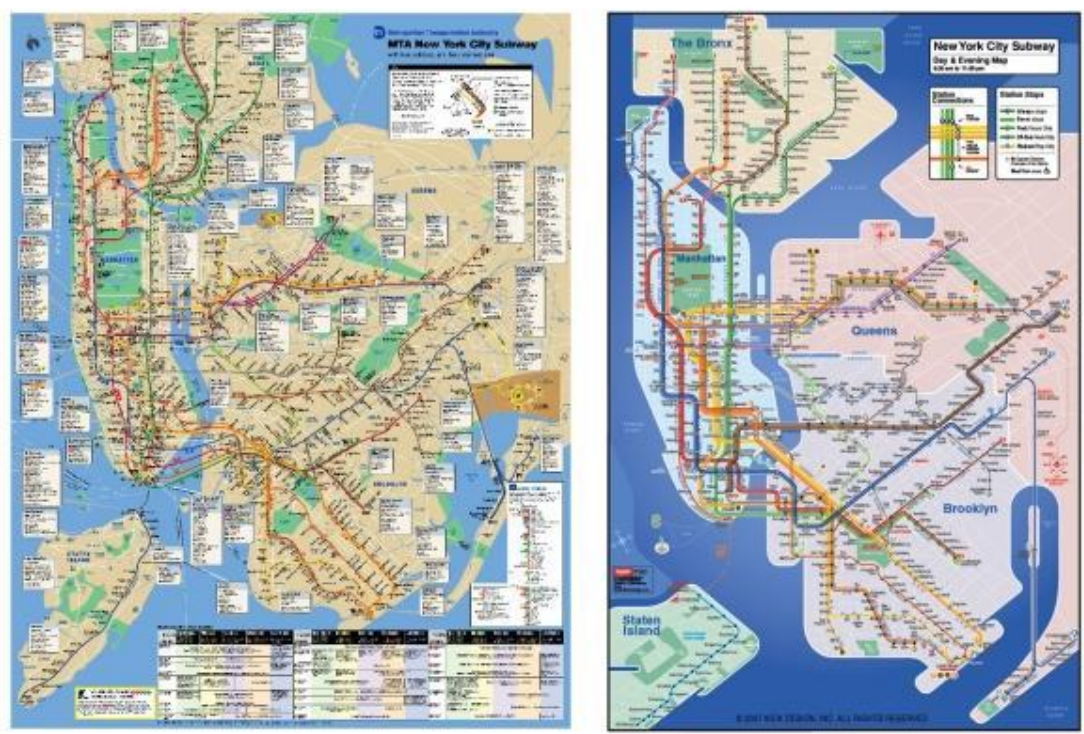

Read Article:

http://www.railway-technology.com/features/featuretransport-apps-key-trends-andinnovations-4323893/

\section{Smart communication for intelligent transport}

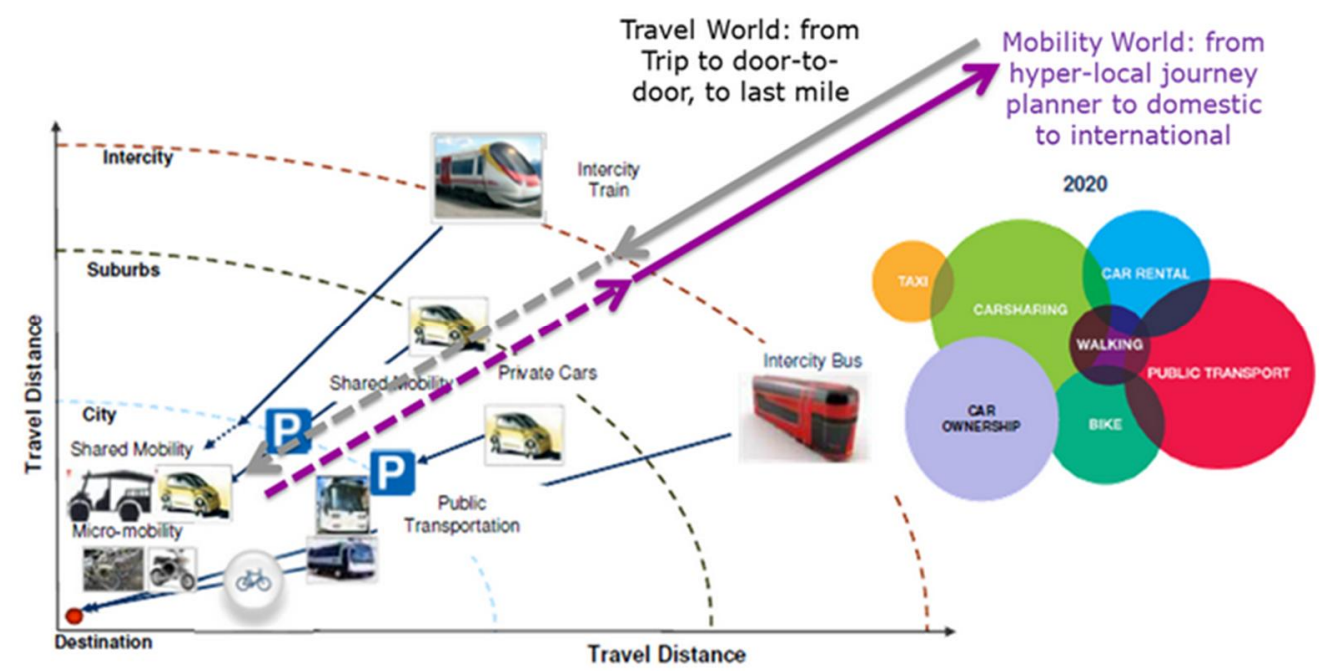

Read Article:

http://archive.ericsson.net/service/internet/picov/get?DocNo=11/28701-

FGB101108/2\&Lang $=$ EN\&HighestFree $=Y$ 
The New Transportation Ecosystem and the Role of Silicon Valley

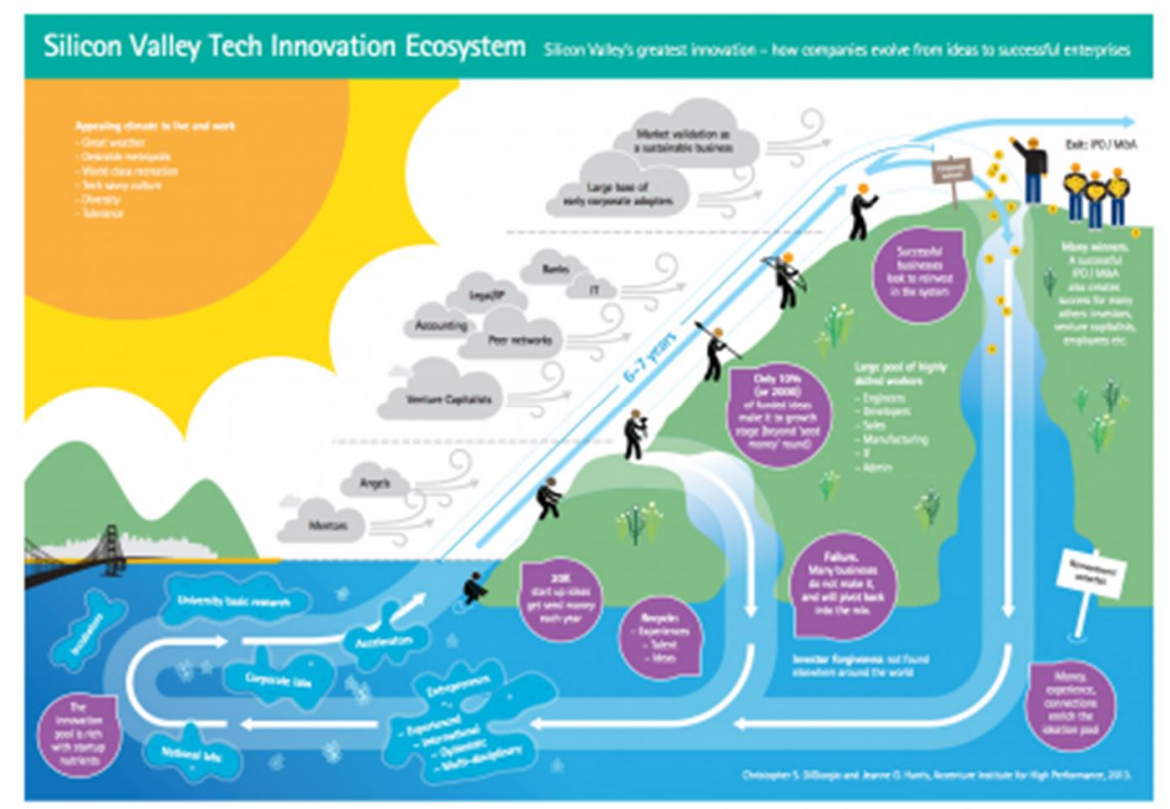

Read Article:

http://siliconvikings.com/blog/2015/7/20/the-new-transportation-ecosystem-and-the-roleof-silicon-valley

What the rise of the sharing economy means for transport

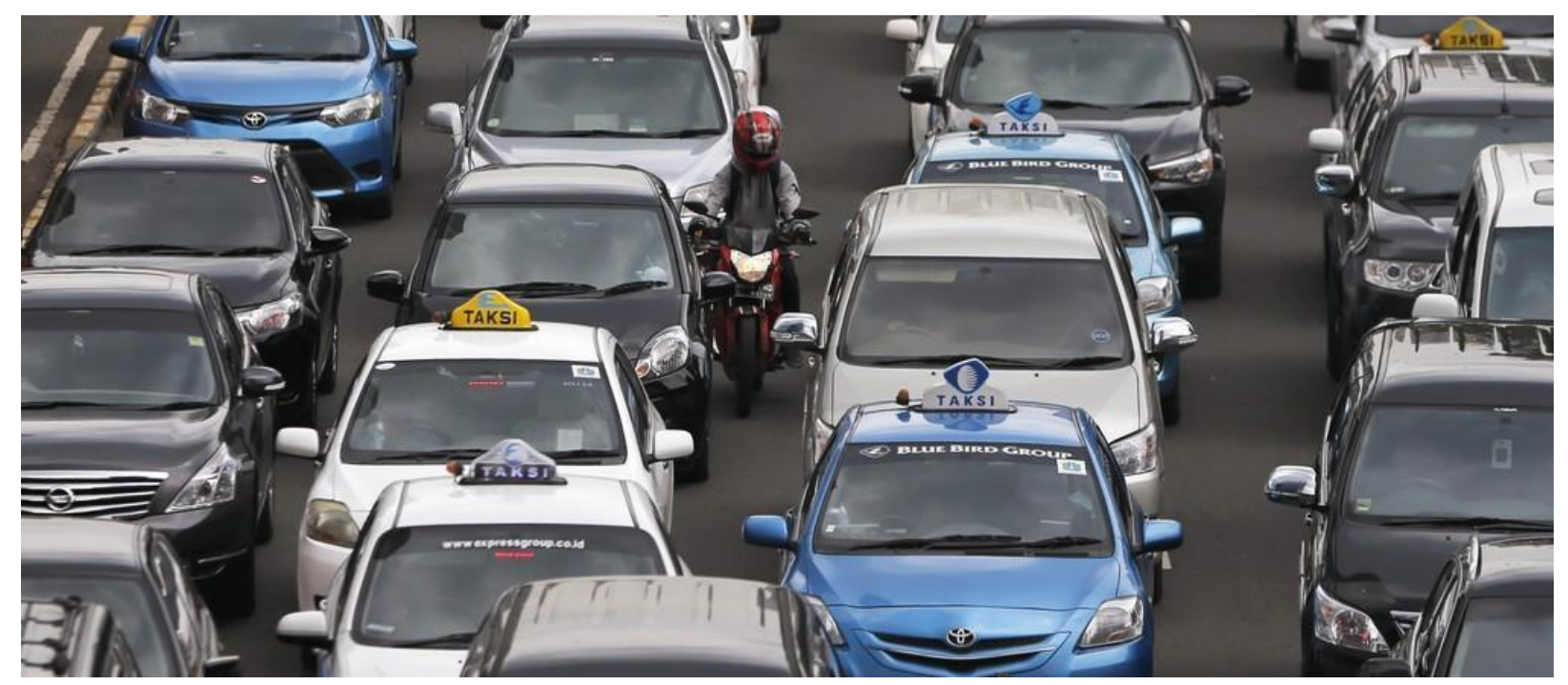

Read Article:

http://2015.internationaltransportforum.org/shared-economy 
Revolutionizing the Travel and Transportation Ecosystem

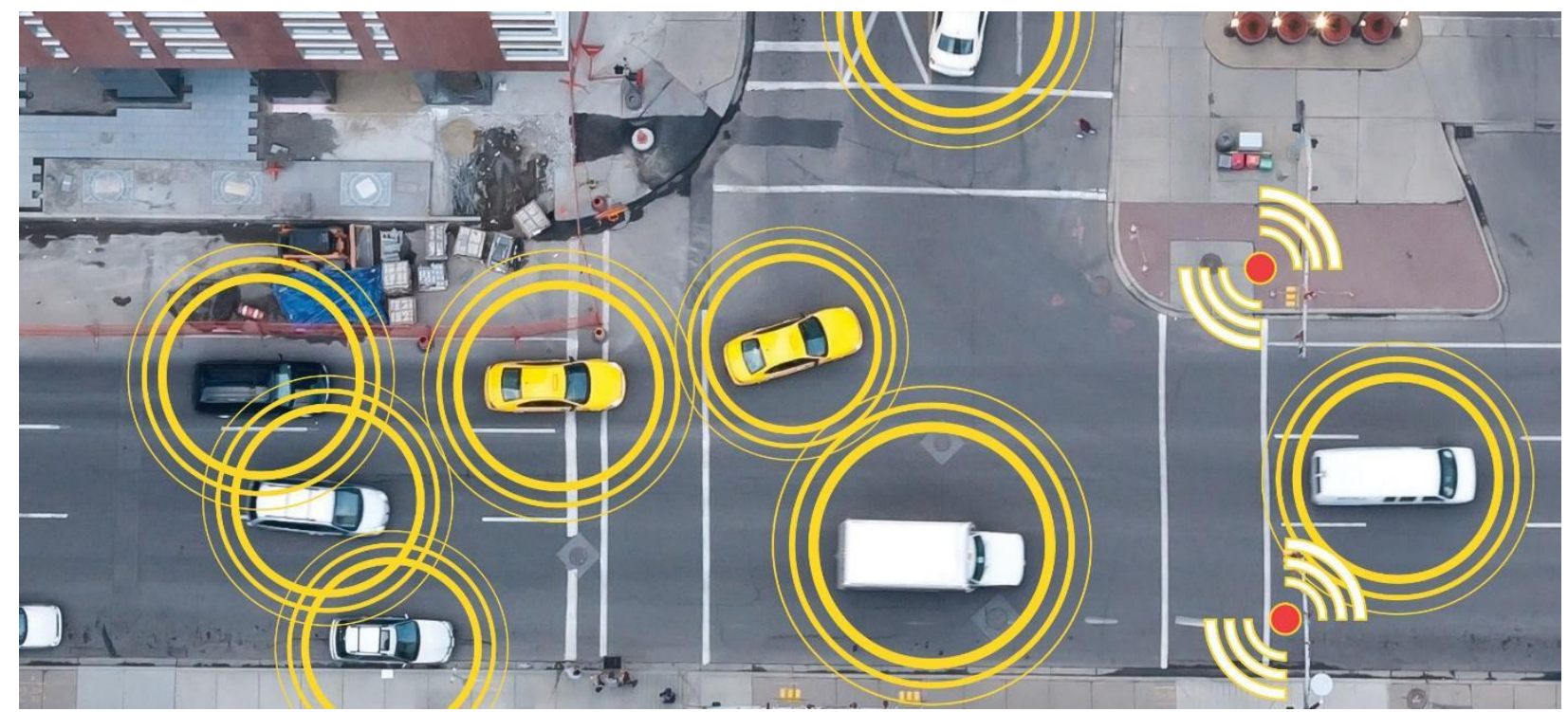

Read Article:

https://www.bcgperspectives.com/content/commentary/travel_transportation_tourism_su pply_chain_management_mei_pochtler_revolutionizing_travel_transportation_ecosyste $\mathrm{m} /$

\section{How Technology is Changing Traffic Reporting}

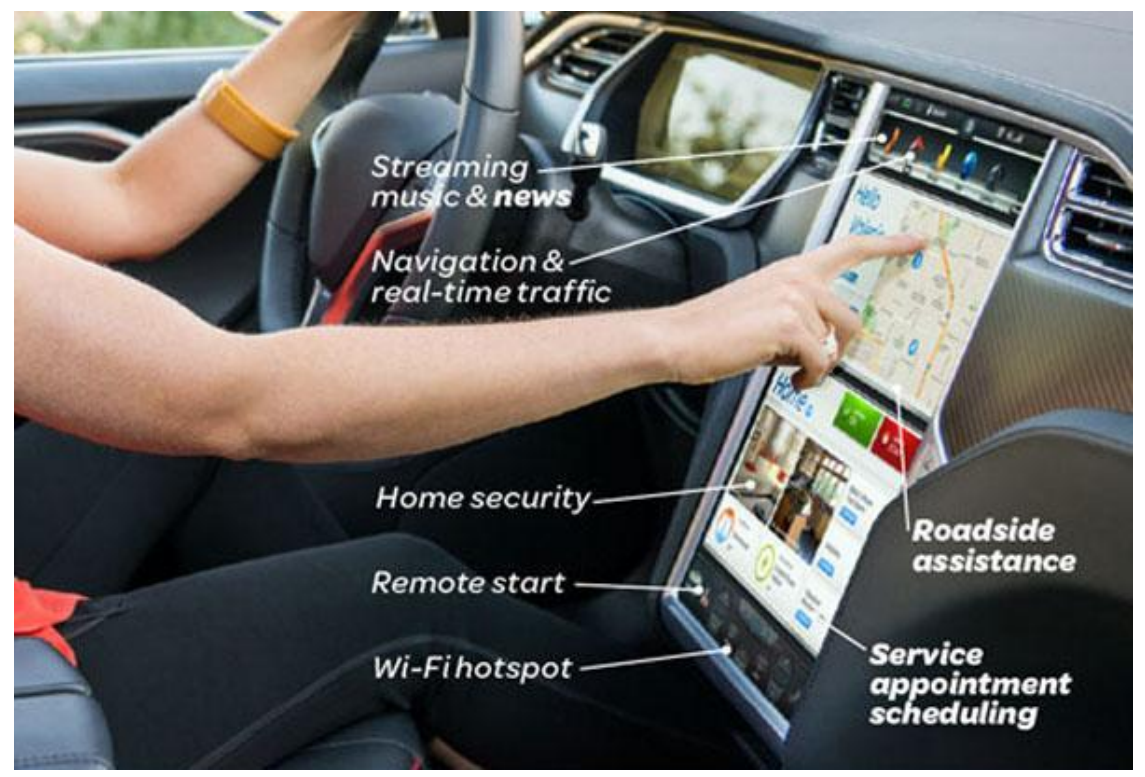

Read Article:

http://business.weather.com/blog/how-technology-is-changing-traffic-reporting 
Arctic testing ecosystem for intelligent transport opens in Finnish Lapland

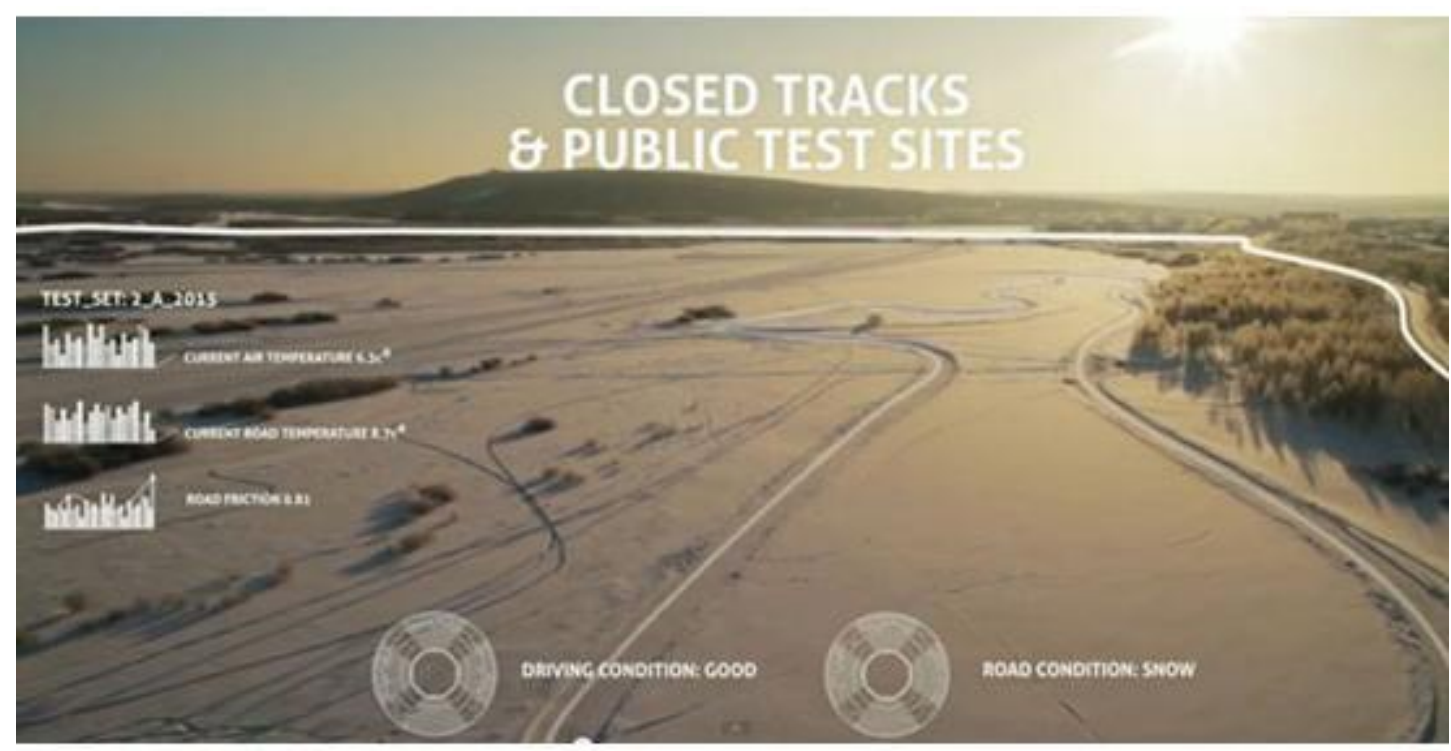

Read Article:

http://www.traffictechnologytoday.com/news.php?NewsID=77169

Intelligent transport - a trump card for Finnish exports

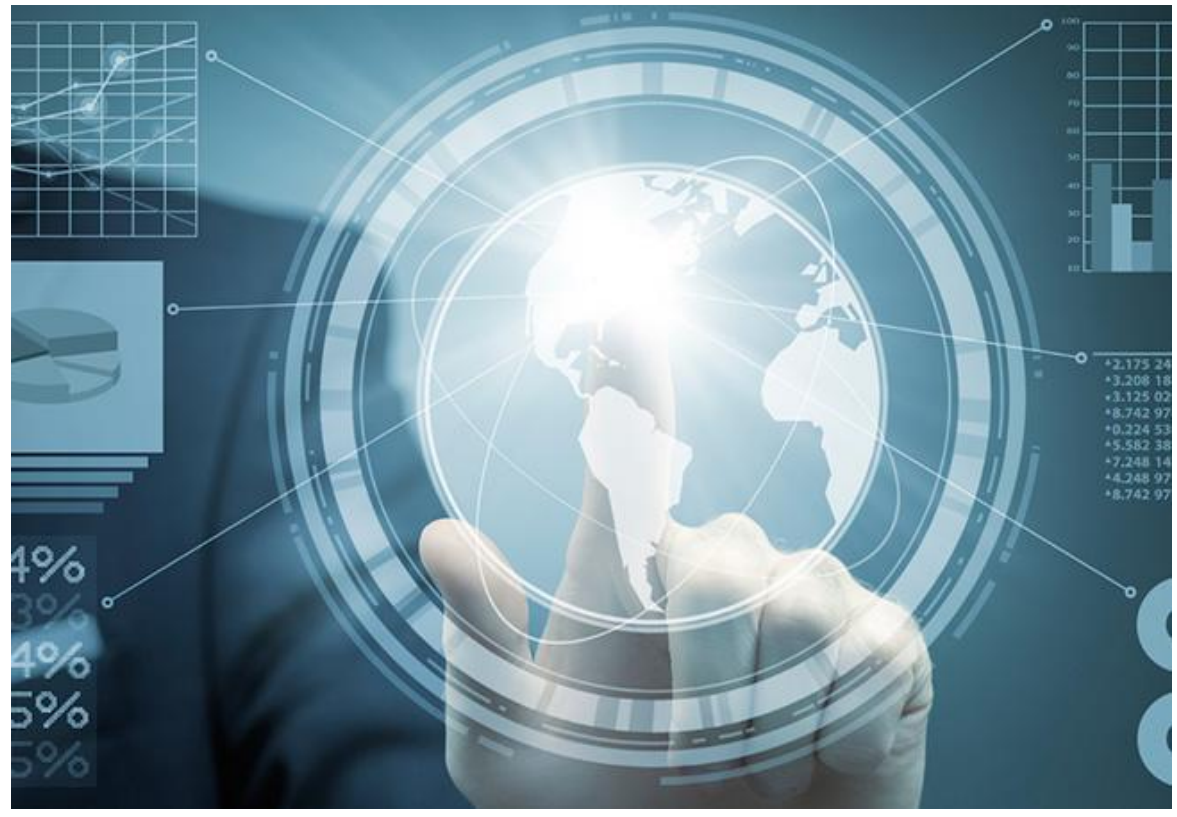

Read Article:

http://www.vttresearch.com/Impulse/Pages/Intelligent-transport-a-trump-card-for-Finnishexports.aspx 
Challenges to Urban Transport Sustainability and Smart Transport in a Tourist City: The Gold Coast, Australia

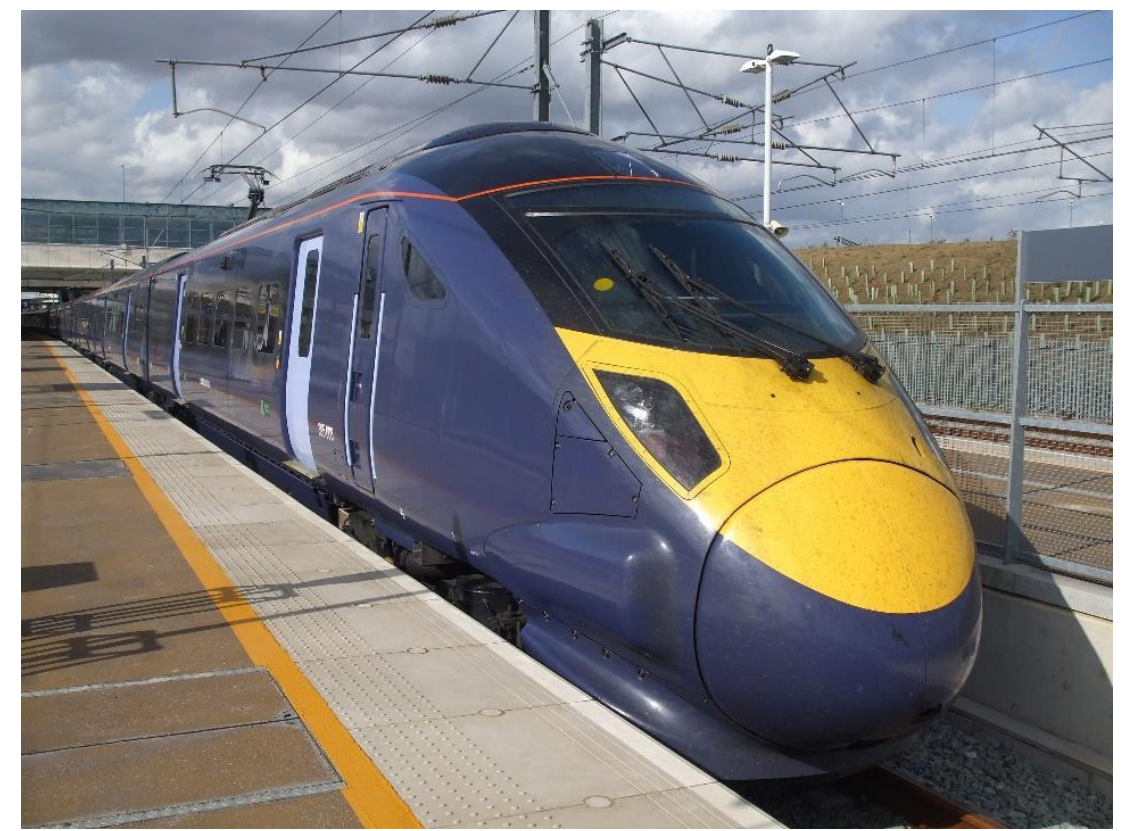

Read Article:

http://eprints.qut.edu.au/15425/1/15425.pdf

Urban Ecosystems: Roads and Transits

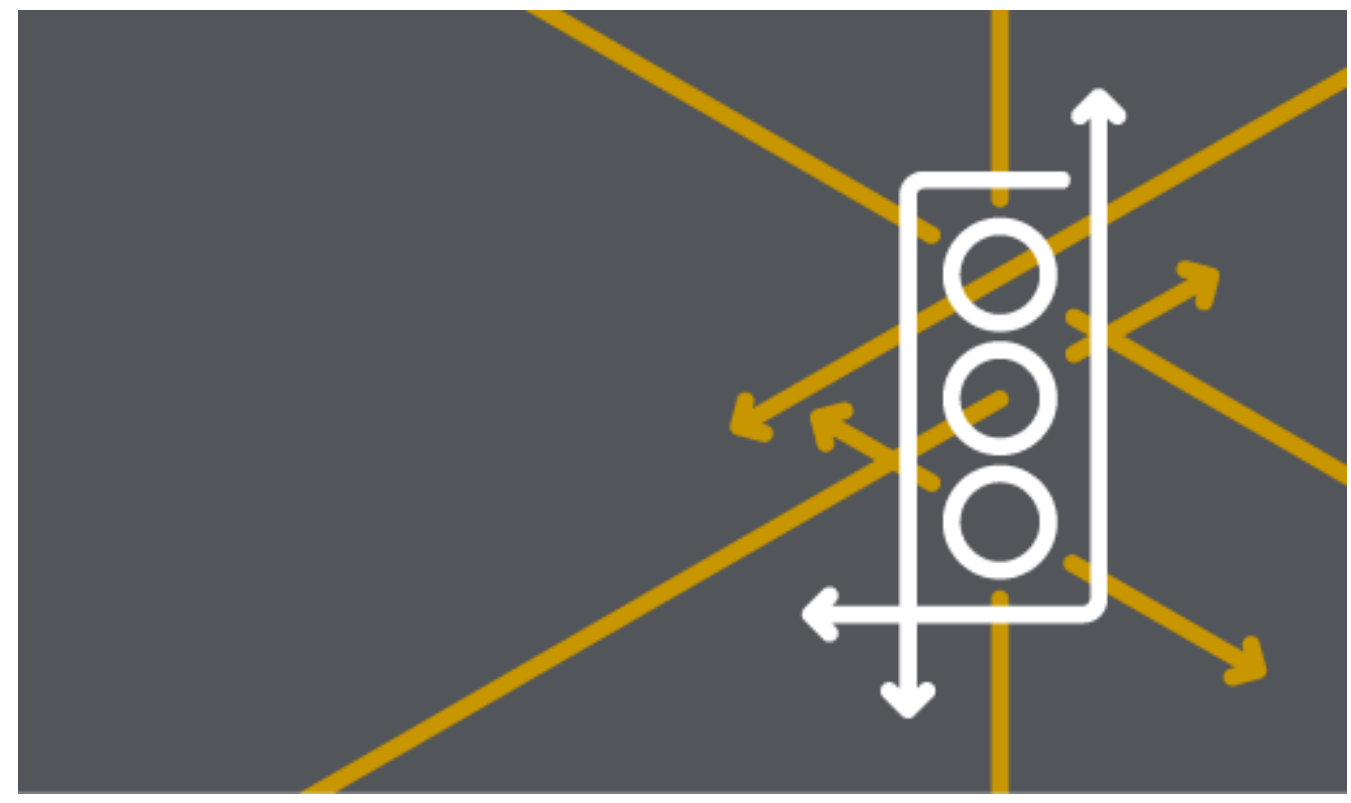

Read Article:

http://citigroup.com/citi/citiforcities/roads_transit/index.htm 


\section{DIGITAL TRANSFORMATIONS IMPACTING THE ECOSYSTEM OF FLIGHT}

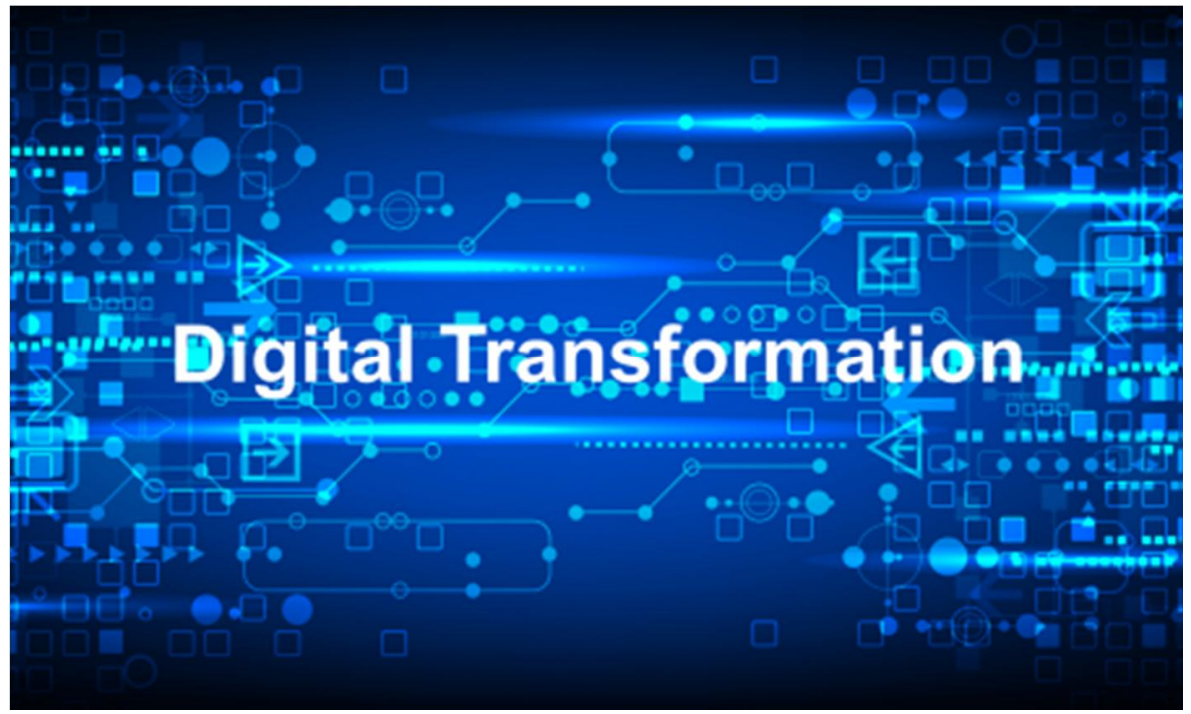

Read Article:

HTTPS://WWW.THALESGROUP.COM/EN/UNITED-STATES/MAGAZINE/DIGITALTRANSFORMATIONS-IMPACTING-ECOSYSTEM-FLIGHT

On Multi-Agent Based Urban Rail Transport Ridership Forecast System

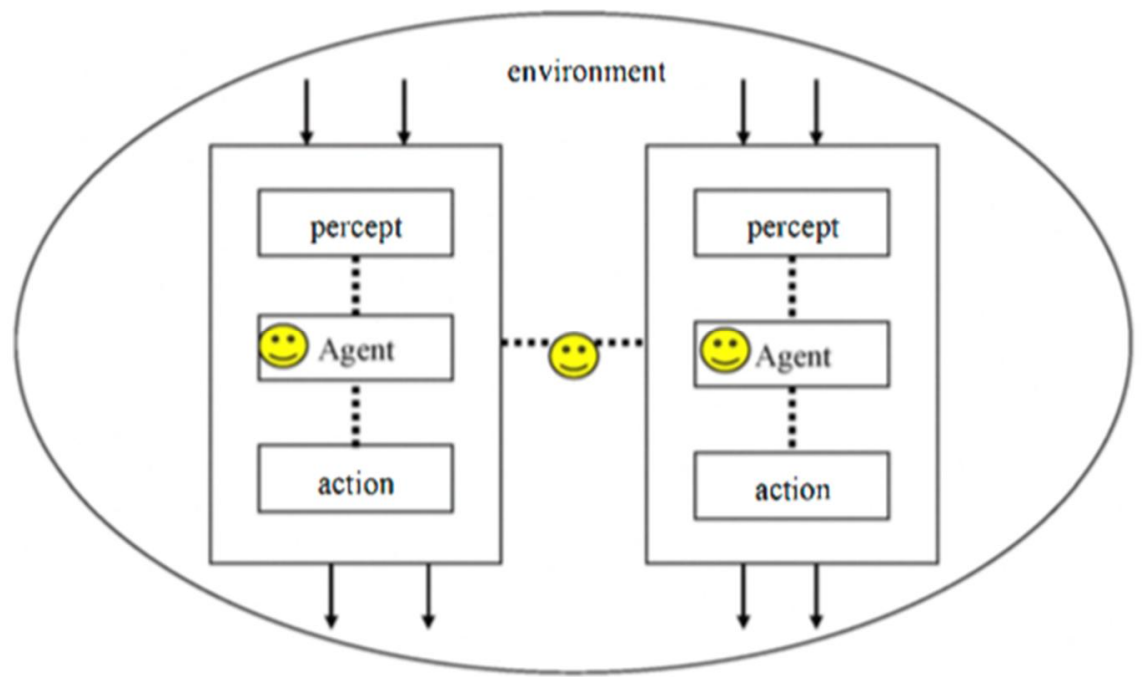

Read Article:

http://cstm.cnki.net/stmt/TitleBrowse/KnowledgeNet/ZNXX201004002071?db=STMI8515 


\section{The digital transformation of transportation}

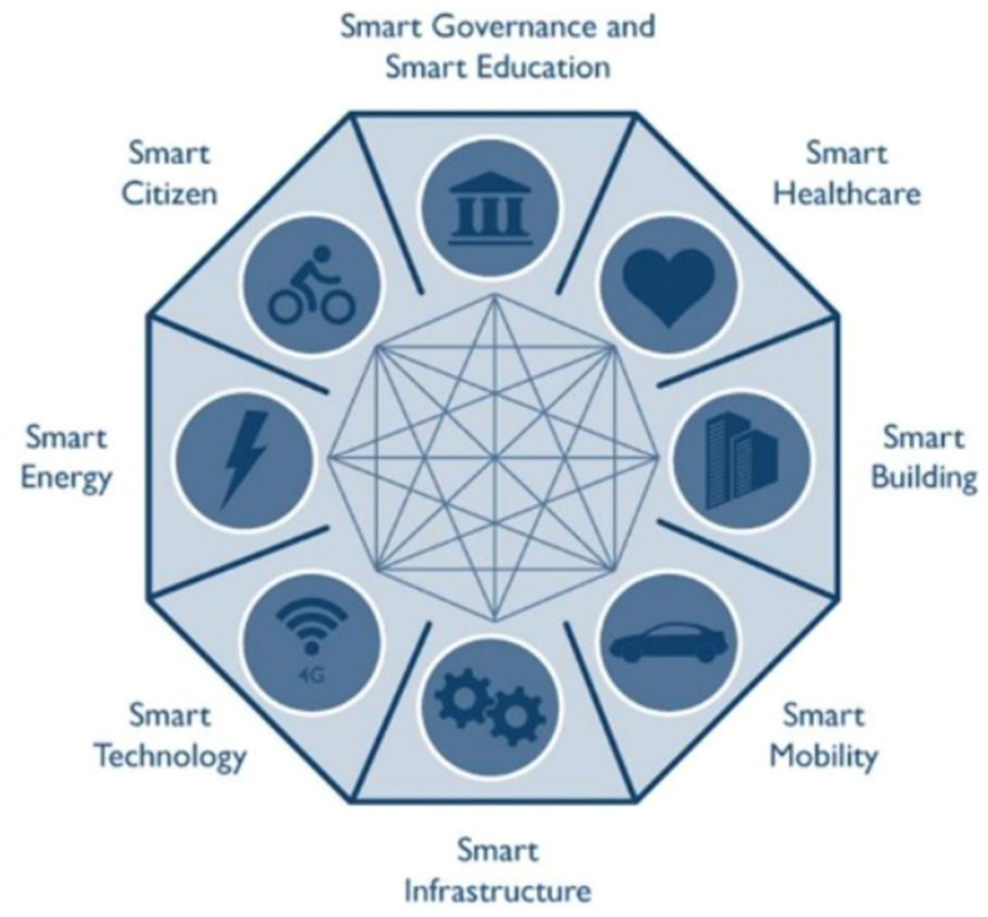

See Presentation:

http://www.slideshare.net/welkaim/the-digital-transformation-of-transportation

Routes to prosperity: How smart transport infrastructure can help cities to thrive

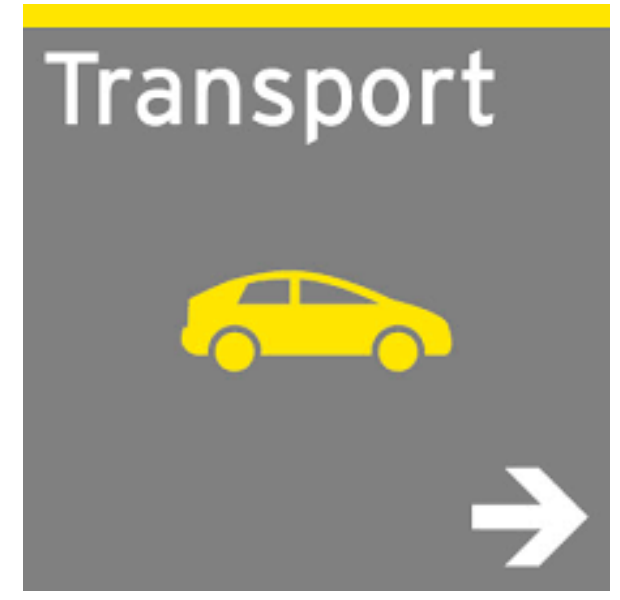

Read Article:

http://www.ey.com/Publication/vwLUAssets/EY-routes-to-prosperity-via-smarttransport/\$FILE/EY-routes-to-prosperity-via-smart-transport.pdf 
Intelligent Urban Exchange - Intelligent Transport

Transportation \& Mobility - Bus Service Planning

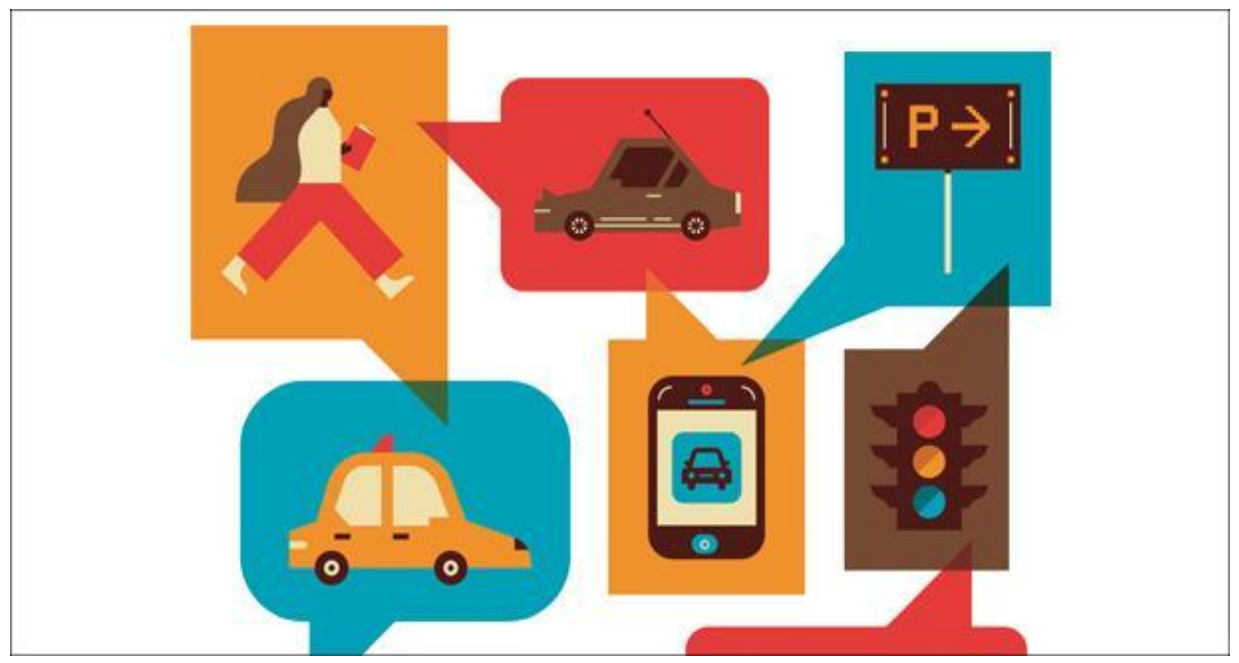

Read Article:

http://www.tcs.com/digital-software-solutions/Pages/Intelligent-Urban-ExchangeIntelligent-Transport.aspx

\section{A system dynamic approach to planning of resilient} urban systems

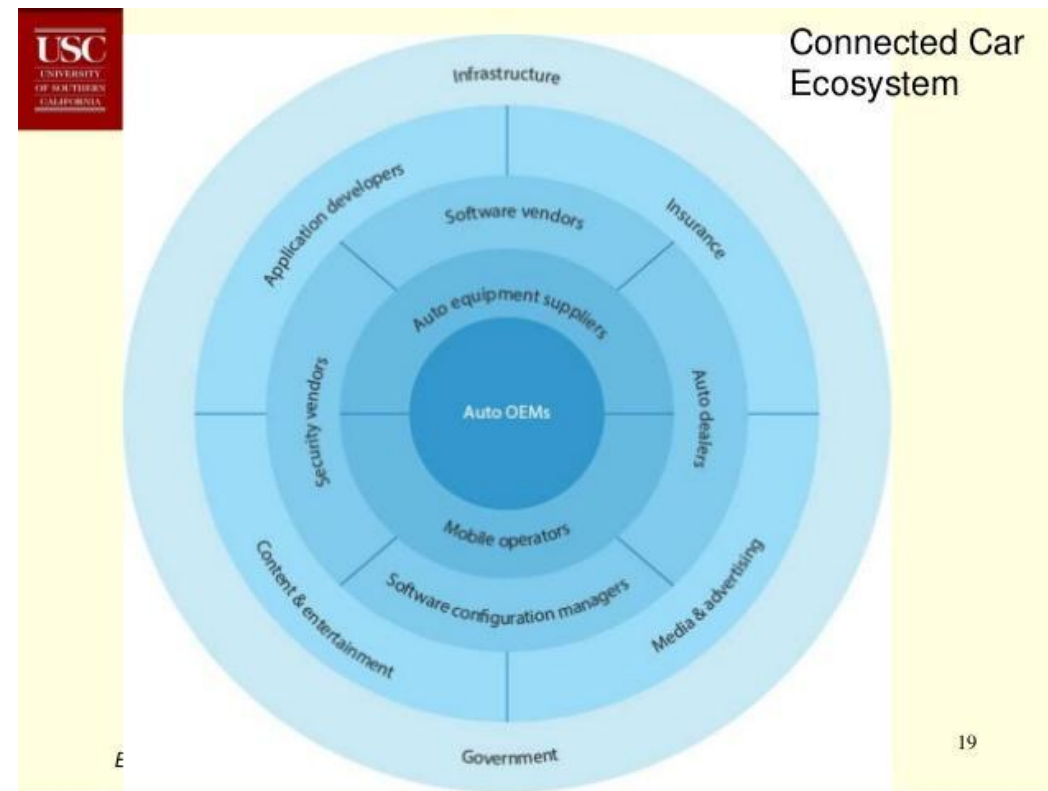

Read Article:

https://itsleeds.wordpress.com/2015/06/25/a-system-dynamic-approach-to-planning-ofresilient-urban-systems/ 
Can the 'internet of moving things' end traffic jams?

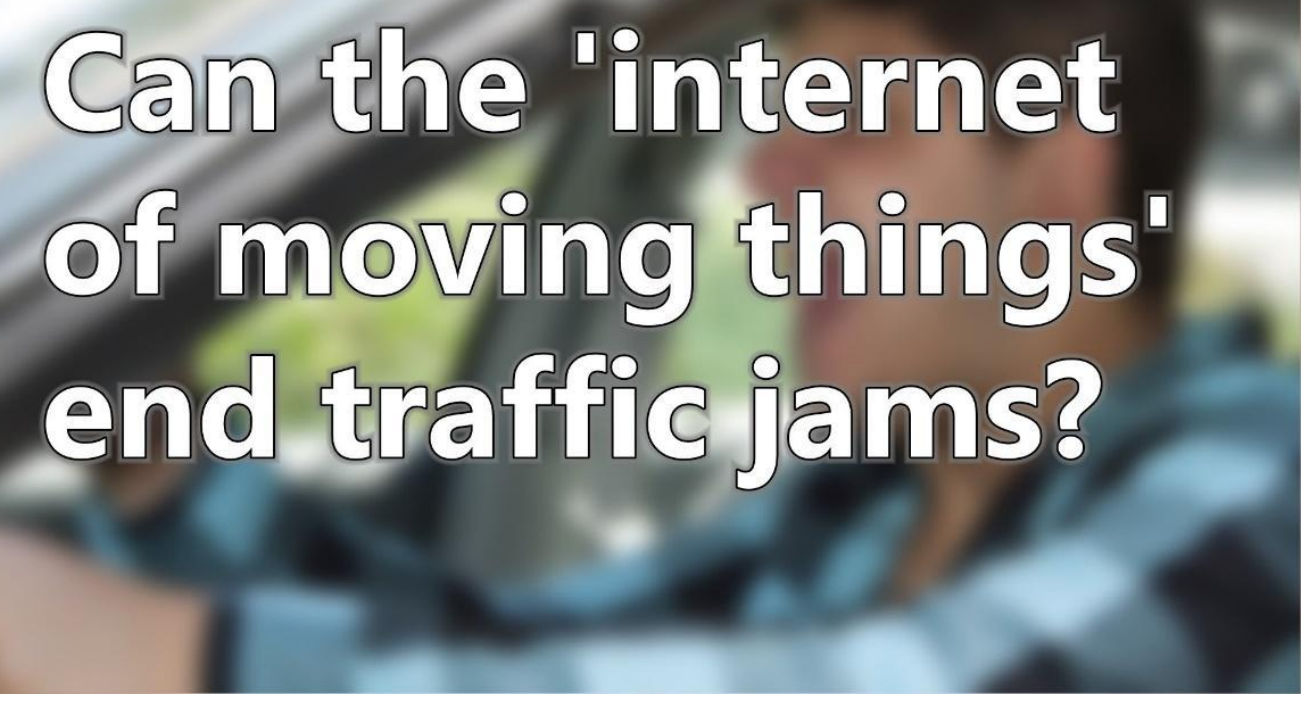

Read Article:

http://www.bbc.com/news/business-36215293

City transport needs saving from itself - here's how to do it City Centre mode share:

2010

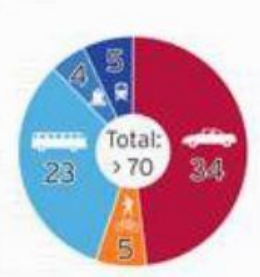

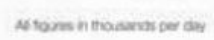
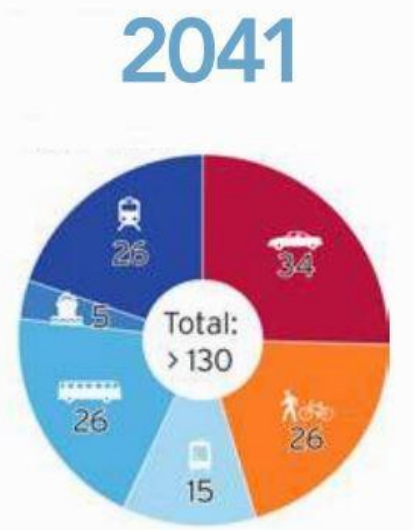

20102041

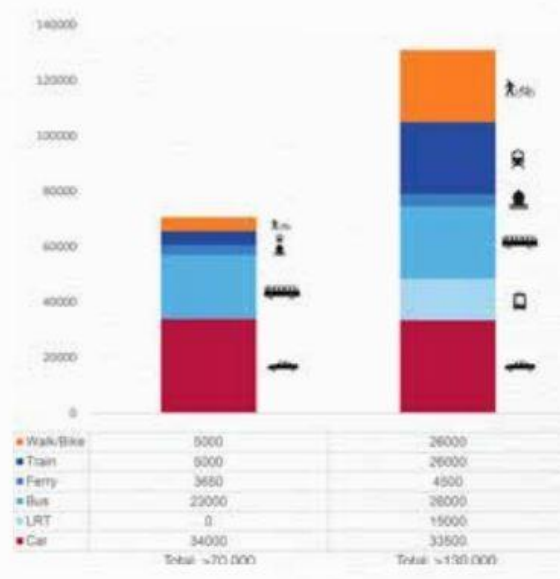

Read Article:

http://theconversation.com/city-transport-needs-saving-from-itself-heres-how-to-do-it45353 
Microscopic dynamic simulation model for pedestrian-vehicle mixed traffic

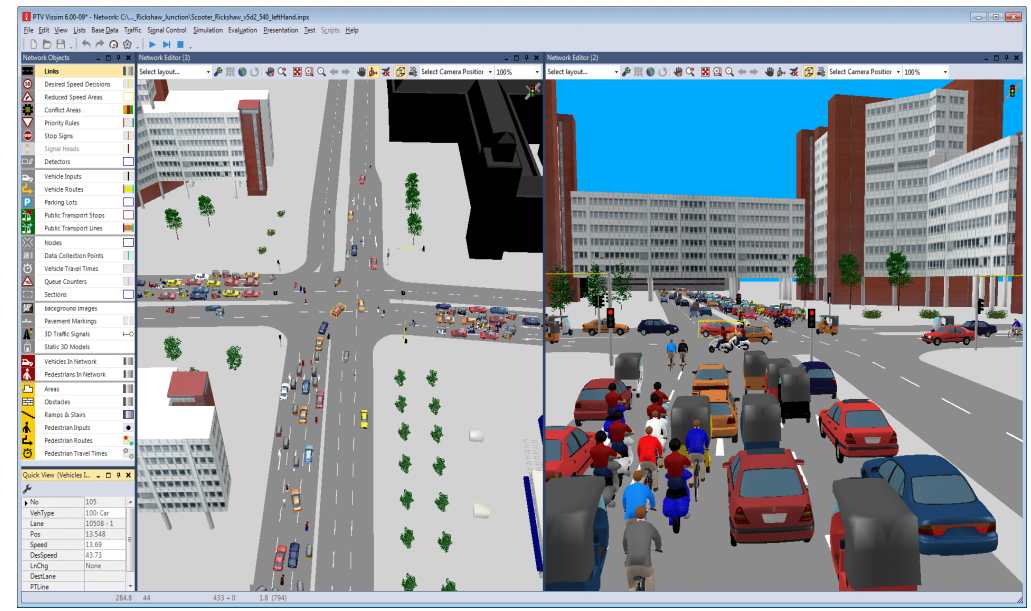

Read Article:

https://www.researchgate.net/publication/251932359_Microscopic_dynamic_simulation_ model_for_pedestrian-vehicle_mixed_traffic

Digital Technology, Mobility And Convenience Services: What Are The Challenges When Building The Collaborative City Of The Future?

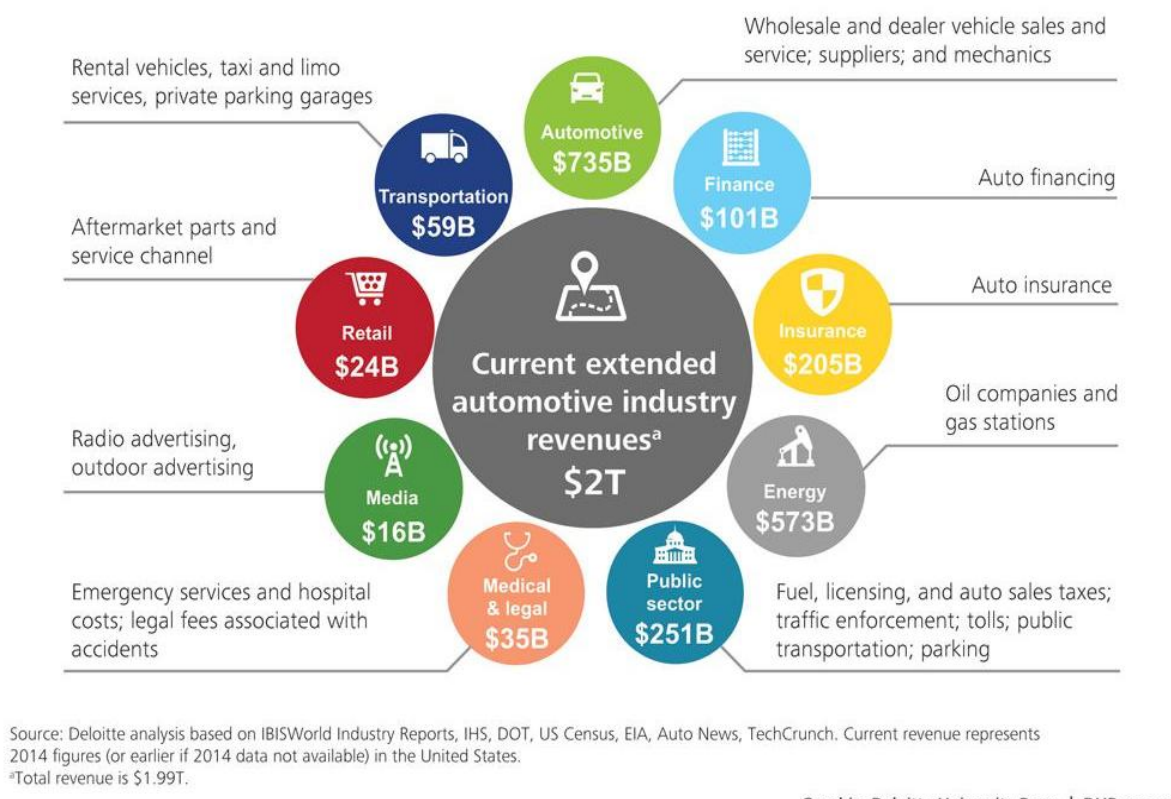

Graphic: Deloitte University Press | DUPress.com

Read Article:

http://www.bouygues-immobilier-corporate.com/en/content/digital-technology-mobilityand-convenience-services-what-are-challenges-when-building 


\section{An Architectural Framework and Enabling Wireless}

Technologies for Digital Cities \& Intelligent Urban

Environments

The Digital City Framework

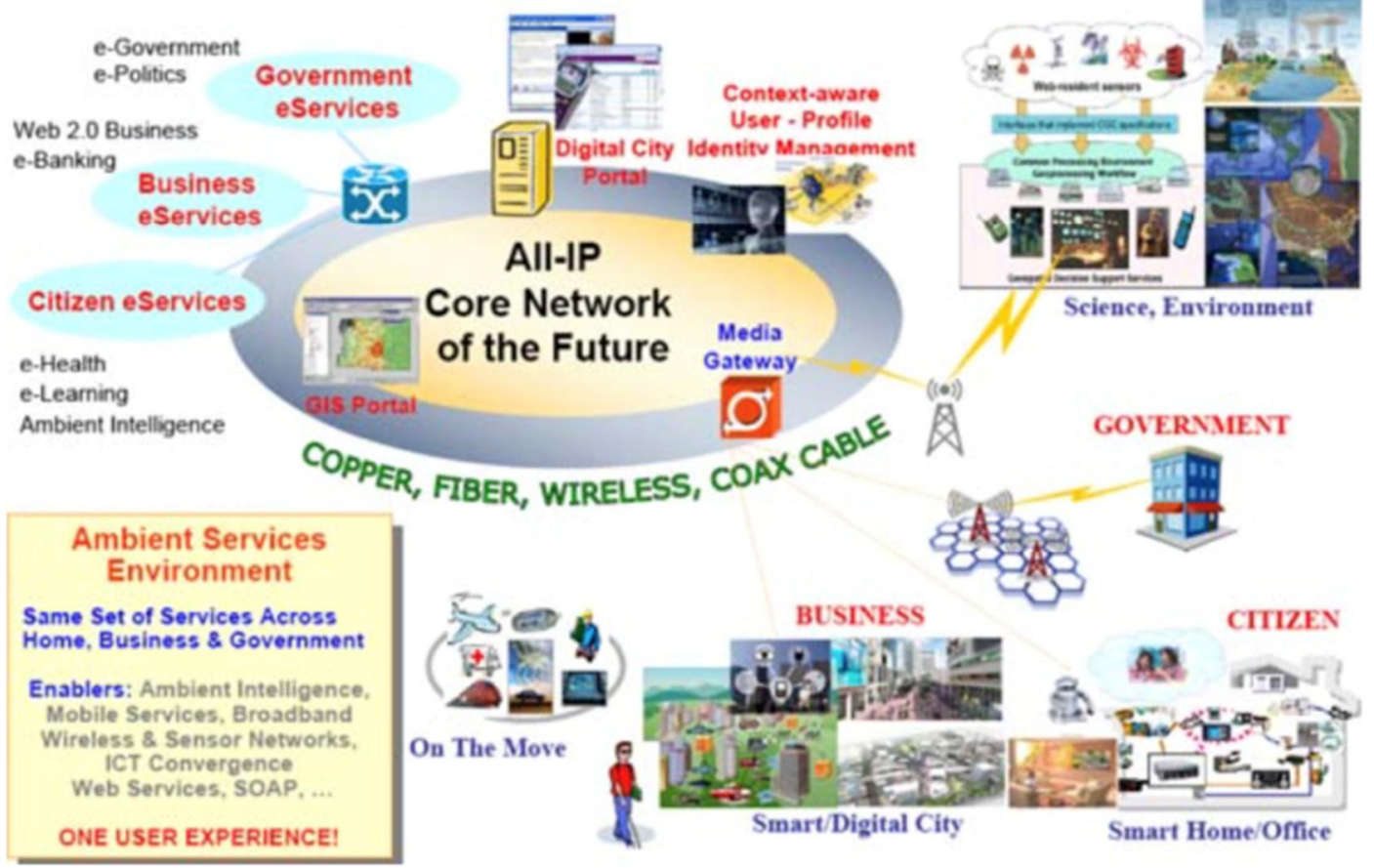

Read Article:

http://link.springer.com/article/10.1007/s11277-009-9693-4 
MaaS Finland aims to revolutionize the global transportation market

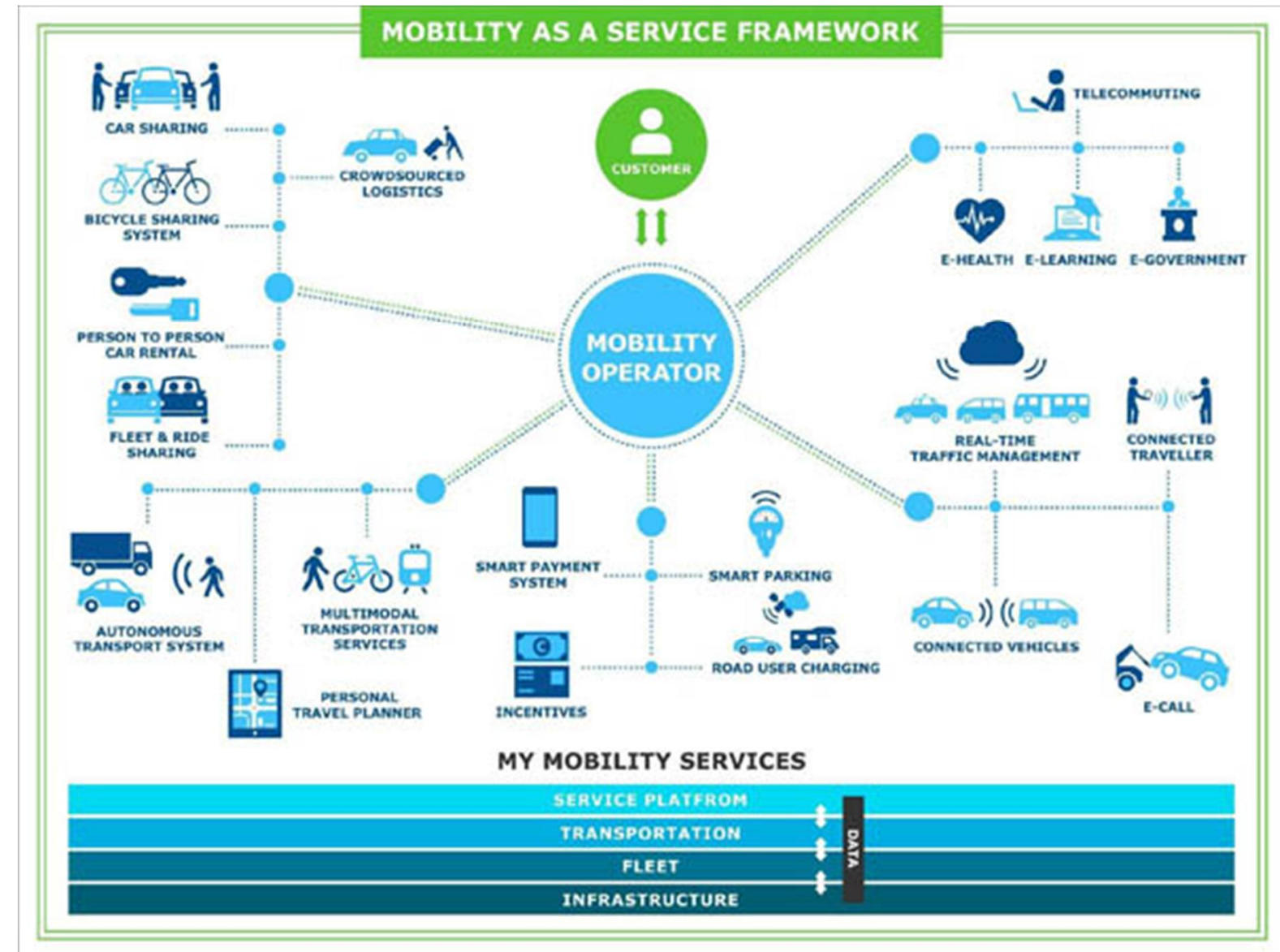

Read Article:

http://www.traffictechnologytoday.com/news.php?NewsID=77471 
Informaatioteknologian tiedekunnan julkaisuja

No. 27/2016 